\title{
Detecting CHANGES IN COGNITIVE LOAD THROUGH AUDIFIED EEG
}

\author{
by

\section{Roderick Spender}

\author{
A thesis submitted to the \\ Faculty of Engineering and Applied Science \\ in conformity with the requirements for \\ the degree of Master of Applied Science
}

\author{
Queen's University \\ Kingston, Ontario, Canada
}

April 2021

Copyright (C) Roderick Spender, 2021 


\begin{abstract}
Concussion is an increasing concern in society, especially with the popularity of contact sports. New research continues to show the dangers of letting concussions go undetected and untreated. Current assessment methods are lacking in their reliability to detect concussions and track healing. Electroencephalography (EEG) consists of data measured from electrical signals from the brain that can give insight into the activity and health of the brain. It would benefit many to achieve an objective assessment method for concussion. The first step in assessing concussion through EEG is to understand the signal properties while performing different cognitive tasks. While these signals are often displayed graphically, they can be converted to sound (audification) to translate the data into a more intuitive medium. By using EEG to understand how the brain processes information under different levels of cognitive load and interpreting this data through audification, this research can pave the way for audified EEG being used to assess brain health, specifically concussion. Untrained participants were asked to differentiate between high and low cognitive load by listening to audified EEG data relating to different tasks. The data were conveyed in ten-second audio samples; each related to tasks of varying cognitive demand. Eightysix percent of participants were able to detect the difference between high and low cognitive load, when listening to a total of sixty-four audified samples of EEG data.
\end{abstract}


This study is presented as a proof of concept that shows audified EEG can be used by novices to differentiate between high and low cognitive load. This approach provides initial evidence to support the theory that EEG could be used to objectively detect changes in brain activity due to concussion. 


\section{Co-Authorship}

This thesis contains the original work of Roderick Spender under the supervision of Dr. T. Claire Davies and Dr. Shane Pinder. 


\section{Acknowledgments}

To my supervisor, Claire: Thank you for giving me a chance and welcoming me into your lab group. You have pushed me in ways that I couldn't expect and helped me gain confidence in my abilities whilst motivating me to take advantage of every opportunity afforded to me.

To my other supervisor, Shane: Thank you for your inspiration and instruction for every step of the design. Thank you for helping a rusty electrical engineer become slightly less rusty.

To everyone in BDAT Lab: Thank you for all your input and advice on various iterations of my approach to this thesis. A special thank you to Stephan Dobri for always providing a fresh perspective and your expertise on data analysis.

To Amanda Melanson: Thank you for helping me collect data on myself when we were in lock-down. I really needed the extra pair of hands to strap electrodes to my scalp.

To Lois and Steph: Thank you for proofreading and for making me laugh during the editing process.

To all my friends and family: Thank you for your continued support and listening to my confusion and/or excitement about the progress I have made.

I would like to dedicate this thesis to my dear friend Tim. He was always so 
supportive of everything I chose to pursue and was excited to see where my work would take me. It saddens me that he can't read this, but I know he would be proud of me. Thank you for being in my life. 


\section{List of Abbreviations}

TBI

EEG

SMR

MRI

fMRI

CAT

$\mathrm{Hz}$
Traumatic brain injury

Electroencephalography

Sensorimotor response

Magnetic resonance imaging

Functional magnetic resonance imaging

Computer aided tomography

Hertz 


\section{Contents}

Abstract $\quad$ i

Co-Authorship iii

Acknowledgments $\quad$ iv

List of Abbreviations $\quad$ vi

Contents vii

List of Tables $\quad$ x

List of Figures $\quad$ xi

Chapter 1: Introduction $\quad 1$

1.1 Context and Motivation . . . . . . . . . . . . . . . 1

1.2 Research Objective . . . . . . . . . . . . . . . . . . 3

1.3 Hypothesis . . . . . . . . . . . . . . . . . . . . . 3

1.4 Thesis Layout . . . . . . . . . . . . . . . . . . . . . . 4

Chapter 2: $\quad$ Background 6

2.1 Concussion . . . . . . . . . . . . . . . . . 6

2.1.1 Statistics . . . . . . . . . . . . . . . 6

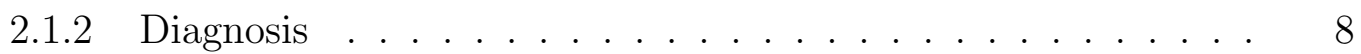

2.1 .3 Recovery......................... 9

2.2 EEG . . . . . . . . . . . . . . . . . . . . . 11

2.2.1 Scalp Location . . . . . . . . . . . . . . . . . . . . 17

2.2.2 Measuring Concussion With EEG . . . . . . . . . . . . . 18

2.3 Cognitive Load . . . . . . . . . . . . . . . . . . . . . . 20

2.3.1 Measuring Cognitive Load . . . . . . . . . . . . . . . . 20

2.3.2 EEG as a measure of Cognitive Load . . . . . . . . . . . . . . 22

2.4 Audification . . . . . . . . . . . . . . . . . 25 
2.4 .1 Parameter Mapping . . . . . . . . . . . . . . . . . . 27

Chapter 3: $\quad$ Methods $\quad 30$

3.1 Preliminary Device Design . . . . . . . . . . . . . . . . . . 30

3.1 .1 Identifying Target User Group . . . . . . . . . . . . . . . . 30

3.1 .2 Design Criteria . . . . . . . . . . . . . . . . . . . 31

3.1 .3 Device Design . . . . . . . . . . . . . . . . . . . . . . 32

3.2 Audifying EEG Signals . . . . . . . . . . . . . . . . . . . 38

3.2 .1 Preparation of Data . . . . . . . . . . . . . . . . . 38

$3.2 .2 \quad$ Matlab Processing . . . . . . . . . . . . . . . . . . . 39

3.2 .3 Amplification . . . . . . . . . . . . . . . . . . . 41

3.2 .4 Filtering and Compression . . . . . . . . . . . . . 44

3.2.5 Audification of the online Dataset . . . . . . . . . . . . 48

3.2 .6 Electrode Location . . . . . . . . . . . . . . . . . . . . 49

3.2.7 Audified Output - Researcher Observation . . . . . . . . . 52

3.2 .8 Time Series Linked to Audification . . . . . . . . . . . . 53

3.2 .9 System Error Detection _... . . . . . . . . . . . . . . 59

3.2 .10 Summary . . . . . . . . . . . . . . . . . . 62

3.3 Survey . . . . . . . . . . . . . . . . . . . . . . . . . . . . 63

3.3 .1 Purpose . . . . . . . . . . . . . . . . . . . . . . 63

3.3 .2 Survey Methodology . . . . . . . . . . . . . . . . 63

$\begin{array}{lll}\text { Chapter 4: } & \text { Results } & 68\end{array}$

4.1 Survey Results . . . . . . . . . . . . . . . . . . . . . . . . . 68

4.1 .1 Inter-Rater Reliability . . . . . . . . . . . . . 70

4.2 Alternative Data . . . . . . . . . . . . . . . . . 72

$\begin{array}{lll}\text { Chapter 5: } & \text { Discussion } & 73\end{array}$

5.1 Audification Process . . . . . . . . . . . . . . . . . 73

5.2 Survey . . . . . . . . . . . . . . . . . . . . . . . . . 73

5.3 Practical Application . . . . . . . . . . . . . . . . 76

5.4 Issues and Shortcomings . . . . . . . . . . . . . . 79

$\begin{array}{lll}\text { Chapter 6: } & \text { Future Work and Conclusions } & 80\end{array}$

6.1 Future Work . . . . . . . . . . . . . . . . . . . . . . 80

6.2 Conclusions . . . . . . . . . . . . . . . . . . . 81

$\begin{array}{lc}\text { References } & 83\end{array}$

$\begin{array}{ll}\text { Appendix A: Background } & 103\end{array}$ 
$\begin{array}{ll}\text { Appendix C: Amplification } & 106\end{array}$

Appendix D: Recruitment Poster 109

Appendix E: Letter of Intent 111

Appendix F: Ethics Submission, Consent Form, and Approval 113

$\begin{array}{ll}\text { Appendix G: Results } & 117\end{array}$ 


\section{List of Tables}

2.1 Observed effects on specific brainwaves and the cause. . . . . . . . . . 24

4.1 Results from Section 1 of the survey. . . . . . . . . . . . . . . . 69

4.2 Average responses from Section 2 of the survey. . . . . . . . . . . . 70

4.3 Inter-Rater reliability depending on values of Kappa. . . . . . . . . . 71

B.1 Examples of EEG technology currently available . . . . . . . . . . 105 


\section{List of Figures}

2.1 A visual representation of brainwaves in terms of frequency bandwidths and their associated behaviours. This example is from Muse ${ }^{\mathrm{TM}}$; a commercial EEG headset company [1]. . . . . . . . . . . . . . . 14

2.2 Layout of the International 10-20 Electrode System [2] . . . . . . . . 18

3.1 A potentiometer is a type of resistor that allows for real time change of resistance. Set up in this way, with another standard resistor, creates a differential amplifier. A differential amplifier will remove noise from both input signals and amplify them, based on a controlled gain. . . .

3.2 The AD210 isolation amplifier uses a chosen gain to amplify the signal to the desired range, whilst removing noise. . . . . . . . . . .

3.3 These test points allow for troubleshooting the initial prototype, and can then be connected to the inputs seen in fig. 3.6. . . . . . . . . .

3.4 An Arduino is programmed from a computer and powered by a dedicated battery. . . . . . . . . . . . . . . .

3.5 A combination of potentiometers allows for testing of the generated signal before combing with the EEG signals. . . . . . . . . . . . .

3.6 Here an earphone output is used, for direct listening to the combined signals in one audio signal. . . . . . . . . . . . . . . . . . . . 
3.7 Circuit schematic for design of the prospective device. . . . . . . . . 36

3.8 Circuit layout for design of the prospective device. . . . . . . . . . . 37

3.9 Visual representation of the task and rest data, as it was recorded for

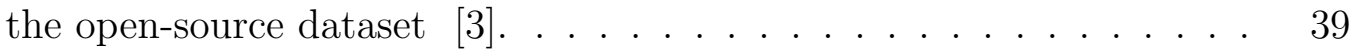

3.10 Demonstrating how the data were extracted from the dataset for use in Matlab. . . . . . . . . . . . . . . . . . . . . . 40

3.11 Amplified EEG data relevant to the rest portion of the motor imagery

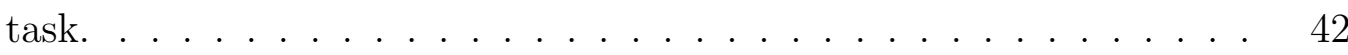

3.12 Amplified EEG data relevant to the motor imagery task. . . . . . . . 42

3.13 Amplified EEG data relevant to the rest portion of the mental arithmetic task. . . . . . . . . . . . . . . . .

3.14 Amplified EEG data relevant to the mental arithmetic task. This data is observed over a slightly longer time because these sections of the data set were recorded over a slightly longer time frame. . . . . . . . 43

3.15 Showing compression of an arbitrary signal. . . . . . . . . . . . 45

3.16 EEG data relevant to the mental arithmetic task filtered into brainwave bandwidths. . . . . . . . . . . . . . . . . 47

3.17 EEG data relevant to the mental arithmetic rest filtered into brainwave bandwidths. . . . . . . . . . . . . . . . . .

3.18 Highlighting the $\mathrm{Pz}$ location that is the focus of the work going forward $[2] \ldots \ldots \ldots \ldots \ldots$

3.19 Amplitude of motor rest data with trough highlighted . . . . . . . . . 54

3.20 Amplitude of mental rest data . . . . . . . . . . . . . . . 55

3.21 Amplitude of motor task data with troughs highlighted . . . . . . . 56 
3.22 Amplitude of mental task data with peaks and troughs highlighted .

3.23 Filtered mental rest data showing comparatively less prominent signals in each brainwave bandwidth . . . . . . . . . . . . . 58

3.24 Filtered mental arithmetic data showing comparatively stronger and more unique signals in each brainwave bandwidth. . . . . . . . . . . . 59

3.25 Erroneous data for motor rest data. . . . . . . . . . . . . . . 60

3.26 Erroneous data for motor imagery task data. . . . . . . . . . . . . 61

3.27 Erroneous data for mental rest data. . . . . . . . . . . . . . . . . 61

3.28 Erroneous data for mental arithmetic task data. . . . . . . . . . . . . 62

3.29 Section 1 of the Qualtrics Survey. . . . . . . . . . . . . 65

3.30 Structure of the survey consisting of section 1 and section 2. . . . . 66

3.31 Question 1, of section 2, of the Qualtrics Survey. . . . . . . . . . . 67

4.1 Showing the percentage of answers selected for each question. Mental Arithmetic (blue) is the correct answer. . . . . . . . . . . . . . 69

A.1 An example of a concussion checklist used by the National Football League. . . . . . . . . . . . . . . . . . . . . 103

C.1 Motor imagery rest data before amplification. . . . . . . . . . . . 106

C.2 Motor imagery task data before amplification. . . . . . . . . . . . . 107

C.3 Mental arithmetic rest data before amplification. . . . . . . . . . . . . 107

C.4 Mental arithmetic task data before amplification. . . . . . . . . . . . 108

D.1 Advert posted on Facebook and Twitter to recruit participants for the online survey. . . . . . . . . . . . . . . . . . . . . . . 110 
E.1 Letter of Intent given to prospective participants of the online survey. 112

F.1 Consent form that was approved by GREB and presented to participants of the survey. . . . . . . . . . . . . . . . . . . . . . 115

G.1 Answers from section 2 of the survey. . . . . . . . . . . . . . . . 118 


\section{Chapter 1}

\section{Introduction}

\subsection{Context and Motivation}

Traumatic brain injury (TBI) is caused by sudden damage to the brain by a blow to the head that is associated with damage to either the frontal, temporal or parietal lobe of the brain. TBI is 44 times more common than spinal cord injuries and 30 times more common than breast cancer [4]. Most of these brain injuries are concussion related. A concussion is a type of TBI caused by a hit to the body or head, causing the brain to move rapidly in the skull potentially damaging brain cells [5].

The most common methods for concussion diagnosis and assessment rely on subjective measures of cognitive function such as memory tests or a checklist of symptoms [6]. These tests are conducted by a trained professional, such as a doctor or sports team medic. Current methods of testing can lead to failed reporting of up to fifty percent of concussions [6] due to misunderstanding of symptoms or intentional misreporting by the injured party $[7,8,9]$. Mis-diagnosis of concussion increases the risk of repeated injury and permanent damage [9]. More accurate diagnostic tools are available, such as Functional magnetic resonance imaging (fMRI), which detects 
change in blood flow of the brain. However, fMRI methods may be inaccessible to patients, especially patients with only a suspected concussion, as fMRI machines are uncommon and very expensive.

EEG is a method of measuring the electrical activity of neuron cells and has been shown to be effective at detecting concussion $[10,11,12]$. There are various tools available for the detection of EEG, ranging from consumer grade to clinical grade, and costing anywhere from hundreds to hundreds of thousands of dollars $[13,14,15$, $16,17,18,19,20,21,22,23,24,25,26,27,28]$. EEG detection is becoming more available, with more consumer grade devices being made for use at home. TBI can alter brain activity [11] which can be seen in EEG data, but it can be extremely difficult to interpret the signal.

One method of displaying information to a user of an EEG system is to audify the signal. Audification is the conversion of an electrical signal into the auditory range [29]. By audifying an EEG signal, the human ear can detect fluctuations in the signal, conveyed by sound [30], rather than relying on complex graphical outputs that require expertise to decipher. It is expected that audifying the EEG signal will allow detection of variations in the brain activity by untrained observers and decrease the time taken for assessment of potential TBI.

Before attempting to assess brain health, it is important to show that the audification of EEG enables the detection of changes in electrical activity of the brain. Changes of electrical activity can be observed by focusing on cognitive load. Cognitive load refers to the amount of working memory that is required to perform a task, imposing on one's mental resources [31]. Low cognitive load refers to a task that is not demanding of mental resources, whereas high cognitive load refers to a more 
demanding task that requires more mental resources to accomplish. Cognitive load is commonly measured using psychometric tests [32, 31], but EEG can be used to measure cognitive load with existing technology [33, 12].

The purpose of this research is to evaluate the use of audified EEG in differentiation of cognitive load by novice observers. Future work will ideally involve the audification of EEG signals of persons who have experienced a concussion to evaluate whether a novice can detect differences in that population.

The motivation behind this work started from research revealing that there is a lack of reliable and accessible diagnostic tools, when it comes to concussion assessment. In looking for effective methods, it became clear that EEG is being used in various avenues but is still exclusive to those with medical expertise. The drive for this research is to show a proof of concept for alternative concussion diagnostic methods that are not only more reliable, but also more accessible to individuals without medical training.

\subsection{Research Objective}

This research was conducted to evaluate the effectiveness of detecting and converting brain signals into audified representations to identify differences in cognitive load by novice listeners.

\subsection{Hypothesis}

Audification will allow the detection of differences between high and low cognitive load, by novices who listen to the signal, without the requirement for extensive training or understanding of graphical representations. If successful this will lay 
the groundwork for using EEG to assess brain health.

\subsection{Thesis Layout}

To address the research objective and evaluate the hypothesis a survey was conducted. The following chapters of this thesis include:

\section{Chapter 2: Background}

Background information is introduced by describing the dangers of concussion, showing the risks and prevalence of mis-diagnosis and leads to a description how audification can provide a solution.

\section{Chapter 3: Methods}

This chapter begins with outlining the original plan to design and construct a device to be used for collection and assessment of our own data. However; when COVID-19 restrictions affected the ability to do human research, the focus changes, and as a result a description of the the process of audification using open-source EEG data as an alternative to a hardware device is described. A description of the data, methods

of audification, and the formatting of the signal to enable files to be used in an online survey are discussed. The online survey assessed the efficacy of audifying differences in cognitive load. This chapter describes the approach and methodology used for the survey.

\section{Chapter 4: Results}

Displaying the results from the survey, this chapter reports percentages and averages of correct answers with a statistical analysis of agreement between participants.

\section{Chapter 5: Discussion}


This chapter discusses the results from the survey and how these results are supported by existing literature, highlighting some issues and shortcomings.

\section{Chapter 6: Future Work and Conclusions}

Future work and conclusions are highlighted within this final chapter. 


\section{Chapter 2}

\section{Background}

\subsection{Concussion}

Concussion can be described as a swelling of the brain caused by trauma or pressure exerted on the brain. Concussion is generally less severe than other brain injuries and is a subset of TBI (traumatic brain injury) [6]. Concussion is often caused by impact from an external object or rapid deceleration, causing the brain to push against the skull [5]. More research is coming to light about the long-term risks of concussion and the dangers of repeated concussion, such as changes in behaviour and overall deterioration of mental health.

\subsubsection{Statistics}

Each day 452 Canadians suffer a serious brain injury [4]. Brain injuries are particularly common in sports, where players are at continued risk of repeated concussion [34]. Concussions in a sporting context have been estimated to account for 300,000

of the 1.54 million mild-to-moderate TBIs that occur each year in Canada [35, 36]. 
Approximately 46,000 concussions were diagnosed by Canadian emergency departments between 2016 and 2017, in youth aged 5-19 [37]. During the same time-frame, over 17,000 sport-related brain injuries were seen in Ontario and Alberta emergency departments. This is an increase of $28 \%$ over the previous five years [38]. It is estimated that as many as 3.8 million concussions occur in the USA per year during competitive sports and recreational activities; however, as many as 50\% of the concussions may go unreported [6]. Kerr (2015) asked athletes to reflect on times they believed they had concussions but a diagnosis was not given after assessment. Kerr (2015) concluded that $21 \%$ of these athletes admitted to not disclosing the injury at all, $91 \%$ deemed their injury not serious, $73 \%$ did not recognize their symptoms at the time, and $73 \%$ did not disclose the seriousness of their injury for fear of being taken out of play/performance [39].

Once diagnoses, concussions may be taken seriously but the overwhelming majority of head injuries are classified as mild traumatic brain injuries and are treated with less concern. Despite these injuries being deemed less severe than a concussion, they can still result in significant cognitive, emotional, and functional disabilities [40]. The severity of TBI is continually underplayed and has only recently been seriously investigated [41].

A concussion has been shown to affect mood and self-esteem in later life [42]. This is particularly common in retired players of contact sports. The incidence of concussion correlates with an earlier onset of Alzheimer's disease in retired football players compared to the general American population [43]. Recurring incidence of concussions can dramatically increase the risk of long-term and permanent damage related to head injuries. Three or more concussions leads to a 2.4 greater likelihood of 
severe depression and higher aggression compared to zero concussions [34]. Retired players with three or more reported concussions had a threefold prevalence of reported significant memory problems compared with retirees without a history of concussion [43]. Retired rugby players, when tested twenty years after their career, showed a higher rate of depression and mild cognitive impairment compared to other sports players [41]. Adults with diagnosed concussion have an increased long-term risk of suicide, showing the need for greater long-term care post-injury [44]. These issues highlight the need for reliable diagnosis and assessment tools that would facilitate sufficient treatments and resting times.

\subsubsection{Diagnosis}

Concussions, especially sport-related, can be difficult to diagnose. Concussions produce a combination of somatic, cognitive and neurobehavioral symptoms that are typically most severe within 24-48 hours post injury [45]. A concussed brain can struggle with regular levels of neural activity and if additional activity occurs before adequate recovery, the brain may be more vulnerable to prolonged dysfunction [6]. Accumulative damage can occur from multiple impacts to the head, even when clinical symptoms are not observed [46]. As a result, it is vital to be able to assess concussion as soon after suspected injury as possible to treat the systems and reduce the likelihood of repeated injury and long-term damage.

Standardized assessment tools provide a helpful structure for the evaluation of concussion, although the efficacy can vary. Clinical practise guidelines [40] and graded symptom checklists allow for objective assessment of the existence and severity

of concussions [6]. The care of athletes with head injuries is ideally performed by 
health-care professionals with specific concussion-focused training.

Recognition and initial assessment of a concussion should be guided by a checklist of symptoms, cognitive evaluations, balance tests and further neurological physical examination. See Appendix A.1 for an example of a checklist used by the National Football League (NFL) [6, 47].

Standardized tests to be used on the side-line of a sports field are useful, but the validity of these tests can vary due to bias [48]. Concussions frequently go unreported due to lack of recognition of symptoms or intentional mis-reporting $[7,8$,

9]. These tests are more reliable when a baseline of expected behaviour is established for the individual being assessed. There are also situational factors of which to be aware whilst conducting side-line tests. For example, balance disturbance is a specific indicator of a concussion despite tests for balance disturbance not being very sensitive. Imbalance tests at the side-line could differ from baseline tests due to differences in surface, footwear, or injuries unrelated to the head. Some symptoms are immediately observable on the side-lines where the injury occurred, but some require continued observation. Medical imaging for athletes is reserved for situations in which there is a strong suspicion of bleeding in the brain [6]. Even if there is no indication of bleeding in the brain, athletes suspected of concussion need to be monitored post-injury for deteriorating condition.

\subsubsection{Recovery}

Once a concussion is diagnosed, current procedure recommends 24-48 hours rest [37] and a one week time-out from play. Recovery is deemed sufficient when the cognitive ability of an individual returns to normal levels, using the same tests that originally 
diagnosed the concussion. The majority of cognitive recovery occurs during the 3-7 day interval post-injury [49]; however, as discussed above, there are ongoing effects. Two-thirds of concussed individuals will regain $80 \%$ of cognitive function within six months [41] of recovery and continue to improve over 18 months [10]. There is also a link between longer resting times and improved cognitive function, whereas increased cognitive activity before appropriate rest leads to a longer recovery from concussion (significantly reducing activity of one's body and brain will aid recovery) [50]. However, it has been shown that $15 \%$ of high school athletes diagnosed with concussion fail to comply with recommended return-to-play guidelines [51]. These findings highlight the importance of continued support, observation, and adequate rest.

Post-concussion syndrome is used to describe a range of symptoms that persist beyond twelve months (after the injury). These symptoms often persist despite a lack of brain abnormalities detected by magnetic resonance imagery (MRI) and computer aided tomography (CAT) scans. On the other hand, quantitative EEG has been shown to be highly sensitive (96\%) in identifying post-concussion syndrome [52].

Sub-concussive events, defined as events that do not result in concussive symptoms, can also lead to motor impairments and cognitive changes especially when these events occur multiple times. Symptoms of sub-concussive events are persistent in up to half of all patients during follow-up assessments conducted at six weeks and six months $[53,54]$.

The aforementioned diagnostic and monitoring tests can have issues in terms of bias, potential interference from a coach, or informed denial from the injured party themselves. While improvements on current assessment methods can be achieved, one issue is that a normally twenty-minute procedure often needs to be extended to 
facilitate continued observation and more detailed diagnosis. Participants in many athletic environments are reluctant or unable to accommodate this extra burden [49]. It is important to minimize the subjectivity involved in assessing a concussion. The subjective element allows tests to be swayed by ignorance or deliberate misreporting. An individual may be able to pretend their symptoms are less severe or non existent, especially if they are familiar with the assessment tools. By developing an assessment tool that is both objective and time-saving, one can minimize bias and maximize detection. This will ensure that the results of a concussion diagnosis are consistent whether or not the injured party (or a third party) is trying to hide or downplay symptoms. One approach that may be effective for objective and immediate assessment is the use of EEG.

\subsection{EEG}

Electroencephalography (EEG) was first detected in 1875 by Hans Berger, who was attempting to discover a mechanism of the brain that would explain extra-sensory phenomena. Clinical EEG was not developed until 1935 [55, 56, 57]. EEG is the method of detecting electrical fields produced by electrochemical signals passing between neurons in the brain. Billions of these signals are required for the electrical field to be powerful enough for detection outside of the brain itself [57]. EEG allows one to gain insight into brain activity as electrical activity of the brain will differ, due to a changing number of neurons firing, depending on the task that the individual is undertaking [58]. EEG is used in the evaluation of dynamic cerebral function and is particularly useful for evaluating patients with suspected seizures and epilepsy $[55,59]$. 
Isolating and using the electrical signals from the brain relies on fundamental circuit theory [58]. EEG can be collected using internal and external electrodes [60]. Internal electrodes are probed directly into the brain and give much higher resolution and less noise because they detect the signal at its source; however, this requires the help of a neurosurgeon and has a higher risk of infection. These factors cause internal electrodes to be more expensive and used less frequently compared to external electrodes. External electrodes can measure the brain activity when applied to the scalp. External electrodes are more common, as they are safer and cheaper. Ideally the location is prepared by being shaved and/or swabbed with alcohol. This minimizes impedance of the signal caused by tissue, bone, and hair that is between the brain and the electrode.

There are two main types of EEG signal used in practise and research: spontaneous responses and continuous rhythms. Spontaneous responses, or evoked potentials, focus on peaks in the signal, associated with specific tasks. One example of this is the N400 signal which results in a large negative amplitude observed 400 milliseconds after a stimulus [61, 62]. Continuous rhythms are known as sensorimotor rhythms (SMR). These are continuous signals observed as changes in relative band power over the sensorimotor cortex and relate to the planning of cognitive tasks. SMR will be the only method of EEG collection discussed in this research.

SMR can be split into bandwidths that have been identified as different brain signals. It is important to understand that these are arbitrary, and the values differ slightly among literature sources.

These brain waves are described and visualised in fig. 2.1 as follows $[1,63,64,65]$ : 
- Delta waves (0.1-4 Hz) are most common during dreamless sleep but are common throughout other actions. Delta waves are more prominent at the temporal lobe.

- Theta waves $(\mathbf{4}-\mathbf{8} \mathbf{H z})$ are dominant in sleep but also common in deep meditation and are more prominent at the frontal lobe of the brain. The amplitude of theta waves increases with increasing mental load.

- Alpha waves $(\mathbf{8 - 1 3} \mathbf{~ H z})$ are most common when awake but best observed when eyes are closed as alpha waves can be saturated by mental effort. These frequencies represent the range for observing the resting state of the brain. Alpha wave power is increased with increasing mental load. Alpha waves are prominent at the temporal, occipital, and frontal lobes during complex tasks.

- Beta waves $(\mathbf{1 3 - 3 0 ~ H z )}$ are observed with increased alertness and focus. Beta waves are used for measurement of mental workload and are more prominent at the temporal and occipital lobes.

- Gamma waves (above $30 \mathbf{H z}-\mathbf{1 0 0 H z}$ ) are observed with high cognitive load, usually information processing that involves communication between different areas of the brain. Gamma waves are associated with the somatosensory cortex and are more prominent at the centro-midline of the brain.

It is important to note that these brainwave bandwidths are arbitrarily decided for classification. All brainwaves discussed can be detected in any part of the brain but some areas allow for more frequency-specific detection of specific brainwaves depending on the type of behaviour being measured. For example, motor movement relates to an increase in theta and gamma waves. In this instance the theta and 
gamma waves would be best detected in the central areas of the brain (the area between the two ears, as shown in fig. 2.2). The amplitude of EEG signals is typically 1-100 microvolts for a healthy brain, although these can be observed at higher levels in certain circumstances, for example, an epileptic brain may display amplitudes an order of magnitude higher [58]. When measuring EEG, amplification must occur to allow for observation. The signal must also be filtered to reduce the effects of electrical artifacts. Artifacts result from other biological signals interrupting the one being measured [66]. Eye [67], sweat gland [68] and muscle activity [69], as well as heartbeat and movement are all elements that affect the signal when trying to

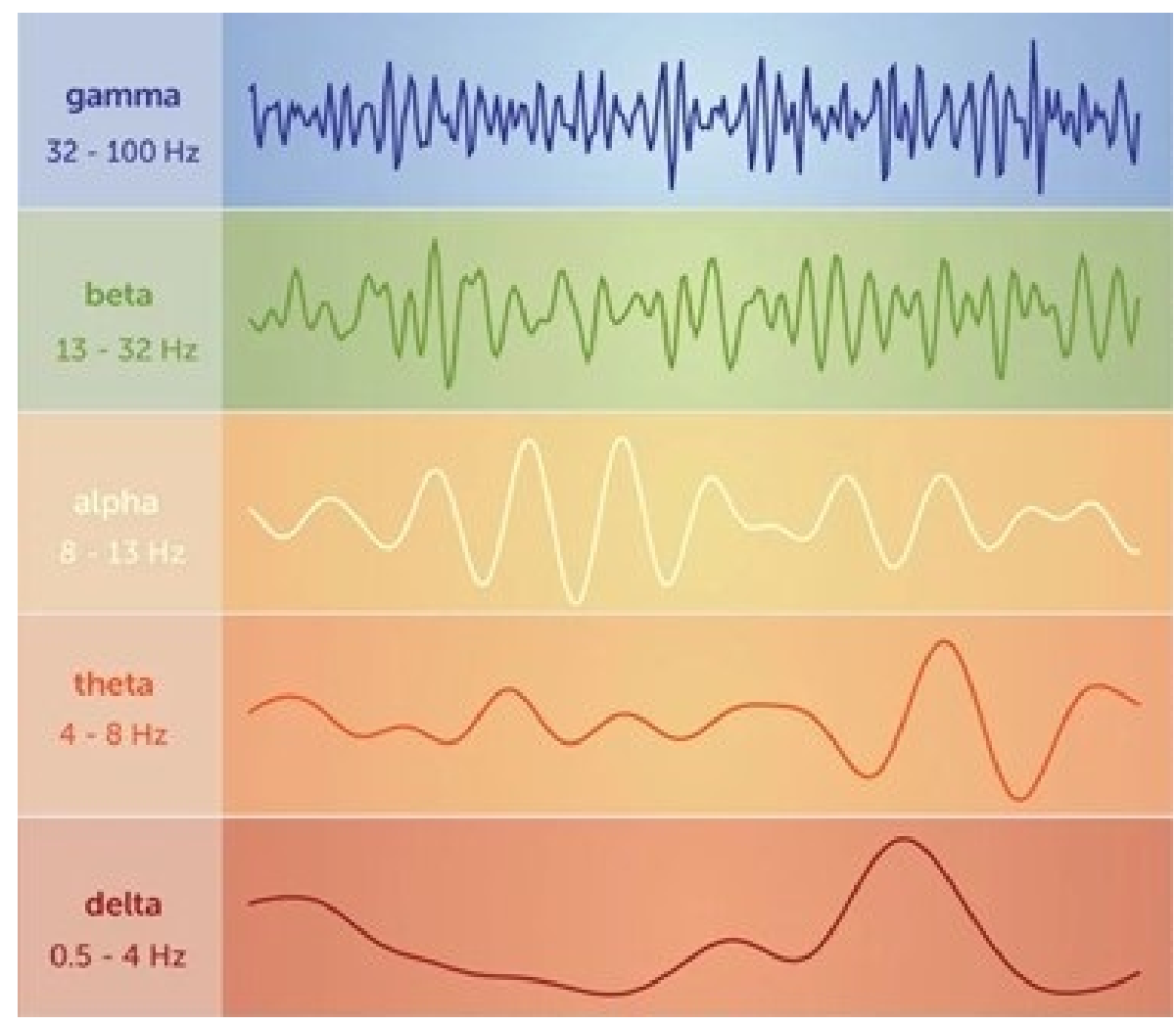

Figure 2.1: A visual representation of brainwaves in terms of frequency bandwidths and their associated behaviours. This example is from Muse ${ }^{\mathrm{TM}}$; a commercial EEG headset company [1]. 
accurately measure EEG. There is also significant noise resulting from background electronic devices, including household power supply outlets. The noise from external sources can be minimized by reducing the number of wires that attach to ground in the circuitry, reducing the lengths of wire, and choosing batteries rather a mains power outlet.

Filtering is performed with internal circuitry or software specific to the device being used. While development of custom EEG systems is feasible, many solutions exist on the market. When using devices currently available, the method of collecting EEG is known, but how it is translated into a usable signal is less obvious. The translation method usually involves some sort of "black box" hardware or software that is unknown to the public, making it harder for people to use the device for research beyond its original purpose, even if it would be beneficial to the client base.

Less expensive devices tend to have a free version of the software or a computer application. There are also ways to access this data with third party software [70], but this assumes the EEG signal collected is reliable. The device may use a method to filter and amplify such that it produces a signal that looks like EEG but is not necessarily a realistic representation of the EEG signals from the user.

Expensive devices require the purchase of specific software. This is understandable under the premise of intellectual property, but it hinders potential research and possible benefits. This black box method of collecting and filtering the data can make it hard to replicate findings among devices. It would be advantageous for a device to collect raw data and facilitate use across various users.

It is suggested that the main reason for the lack of EEG applications to facilitate health monitoring in daily life is the minimal understanding of the concept of EEG 
itself and the current reliance on specialists [71]. EEG is a tool derived from multiple specialities, such as electrical engineering and medical practise which are not common knowledge [24]. We believe that by having a user-friendly set-up and translation of data, combined with obvious benefits to one's health, the adoption and application of EEG would have fewer roadblocks.

When looking at tools to measure EEG, it is important to make the distinction between medical grade technologies and consumer grade technologies. The main difference between medical and consumer grade is the data that is collected by the technology. Medical grade technologies can provide data that facilitates suitable treatment whereas consumer grade technologies give us insight into patterns that require further investigation or intervention. There is strong research showing the value of investing in more expensive EEG headsets, but also showing that good quality EEG data can be collected from consumer grade EEG headsets [72]. For reference, a list of available EEG headsets can be seen in Appendix B.1. There are certain issues that are more common with consumer EEG headsets, such as eye twitching artifacts, but these can be minimized, and will only effect readings from the frontal cortex. Consumer headsets have the advantage of reduced set up times and portability. Some users may not have a problem with longer set up times [26] but, in cases of emergency treatment such as concussion (TBI), time is an important factor for diagnosis and treatment [20].

Another distinction between consumer and medical grade EEG headsets is the number of electrodes used. Some less expensive models may only have one electrode, whereas a medical grade system will have dozens of electrodes [72]. Devices can have fixed electrodes or a cap in which the electrode placement can depend on the intended 
measurement purpose. As different areas of the brain are associated with different brain activity, consistent electrode placement is important especially for comparative research and medical purposes. A widely used reference for electrode placement is the international 10-20 system [73].

\subsubsection{Scalp Location}

The international 10-20 system is a universally recognized tool when referring to locations of electrodes on the scalp, and can be seen in fig. 2.2. The 10-20 system facilitates measurement at specific brain locations by having electrodes spaced out at $10 \%$ or $20 \%$ of the total front-to-back or left-to-right measured distance of the skull. This layout allows for consistency among participants when evaluating the effect of different tasks on brain signals. Certain areas of the brain are also associated with different brain frequencies. This study will focus on motor imagery and mental arithmetic tasks. Motor imagery involves a user imagining the movement of a limb or muscle. Mental arithmetic involves problem solving, usually mathematically based questions or puzzles [64, 74].

Motor imagery or movement can elicit signals over central areas, annotated by 'C' $[75,76]$. Vision is best detected over the central areas and posterior areas, annotated by ' $\mathrm{P}$ ' [56]. Auditory function is best recorded over temporal areas, indicated by a ' $\mathrm{T}$ ' [77]. Frontal and fronto-temporal areas, annotated by 'F' and 'FT' are especially susceptible to TBI due to the proximity of the cranial vault [78].

In summary, rhythms appear to reflect the functions of the specific cortical areas from which they originate. The 10-20 system allows us to identify the location of detection, allowing for repeatability and consistency among participants or across 
research studies.

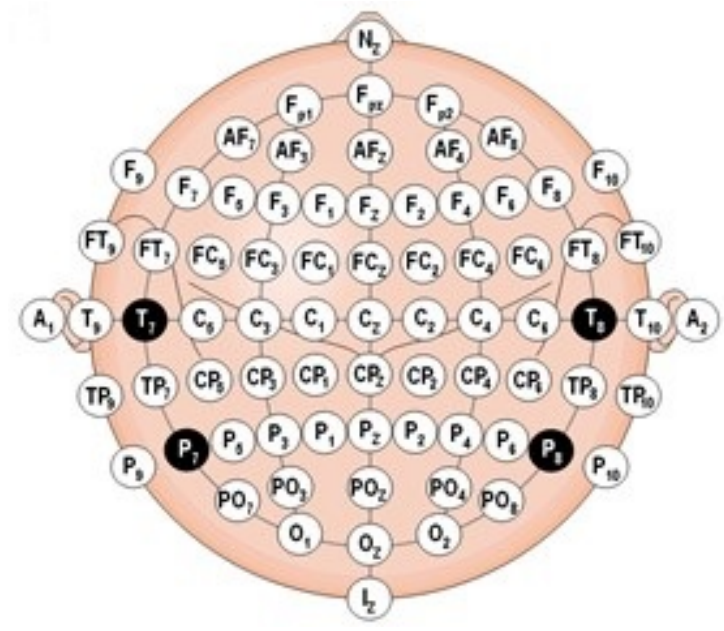

Figure 2.2: Layout of the International 10-20 Electrode System [2].

\subsubsection{Measuring Concussion With EEG}

Research shows that it is possible and effective to assess the existence of a concussion using EEG. A concussion can lead to abnormal patterns when an injured party is assessed with EEG. EEG has been shown to be more reliable than MRI or CT scans, which detect cortical damage such as bleeding or skull fractures; however a concussion may be present without the existence of cortical damage [79, 80, 81, 64]. EEG has been shown to be greater than $90 \%$ accurate in the detection of mild concussive head injuries [82].

Effects on SMR have been observed in concussed individuals to give some EEG based indicators of an existing concussion:

- Alpha wave activity and the higher end of beta activity $(20.5-36 \mathrm{~Hz})$ is less prominent, while lower beta activity $(14-20 \mathrm{~Hz})$ is unchanged [83].

- Amplitude of alpha waves is reduced, but only in posterior regions [84]. 
- Amplitude of delta waves were significantly decreased [64].

- Normally theta bands would differ between regions of the brain, but these differences are not observed in concussed individuals. There was no difference observed between the bands of high alpha and high beta, but there was an overall decrease in amplitude across the SMR spectrum [64].

- Suppression in the range of 7-13 Hz [11].

- Power of the theta band is overall reduced [85].

- The ratio of alpha to theta decreased significantly for the first 10 days, postinjury [86].

The majority of EEG medical applications focus on epilepsy, showing that EEG gives valuable insight into brain health. There is more research emerging using EEG to assess brain health in concussed individuals. EEG assessment for concussion is advantageous compared to the checklist approach as it relies on computer data, making it easy to store the data for later research and assessment. It also minimizes the number of professionals required to assess multiple injured parties as the method can be replicated with minimal training, making the process more efficient [87]. Development of a system to allow EEG collection on-site would be valuable. The system must be portable and robust, with simple but reliable output, ensuring that EEG can be integrated into the current standard procedures for assessment and treatment of concussion [88].

We propose that the use of audified EEG will allow novices to detect differences in brain signals. If effective, this work establishes a proof of concept leading to the use of EEG in the detection of concussed brain function compared typical brain function. 
Before attempting to assess concussion with audified EEG, it is important to show that audification of EEG can be used by novice participants to identify differences in brain function. This thesis will focus on the ability of novice users to identify differences in audified EEG signals between tasks that are of high and low cognitive load. Changes in SMR can be used to measure cognitive load, without the need for an existing injury.

\subsection{Cognitive Load}

The term cognitive load is used to refer the amount of working memory resources that performing a task imposes on the cognitive system [89]. Once the demands of a task exceed mental resources, performance and learning begin to decline [90]. It is theorized that external stimuli are sensed and filtered through sensory memory, before progressing to working memory, where the bulk of critical thinking and decision making takes place [91]. Therefore cognitive load can be defined as the amount of working memory employed for a task [92]. A task analysis is required to translate cognitive load into valid indices of mental effort [93].

\subsubsection{Measuring Cognitive Load}

"Measuring cognitive load has been an area of research for the last thirty years. While self-reported measures of cognitive load are reliable and sensitive to changes in task difficulty, they cannot be employed in real time without interrupting work-flow" - Erika Johannessen (2019) [32].

The definition of cognitive load revolves more around a person's ability to undertake a task than the task itself, meaning that a task performed by two individuals 
may not elicit the same amount of cognitive load [94]. The two most widely used verbal reporting techniques are concurrent and retrospective reporting [95]. Concurrent reporting requires participants to verbalize the amount of effort they believe they are putting into a task; however, potentially interrupting cognitive flow. Retrospective reporting means the assessment of effort is done after the test, relying on memory. Both rely on participants being honest and knowledgeable about the subjective term "effort". Subjective measures such as these can be unreliable because they depend on the user understanding how to convey their cognitive load. Accuracy of reported cognition differs among each user, based on whether they understand the question, or just want to appear less strained $[93,96]$. Defining and measuring cognitive load in increments of low or high is itself an element of subjectivity. It is important to make the measurement of cognitive load as objective and quantitative as possible.

In this research cognitive load will be measured in terms of low and high cognitive load, exhibited by tasks that involve the brain processing motor imagery tasks and mental arithmetic tasks. Carrying out different tasks is a reliable way of eliciting different levels of cognitive load. By having tasks that have different demands, a varying cognitive load can be measured. For example, by not focusing on anything or relaxing, a low cognitive load can be elicited and observed. By undertaking a task such as computing the response to a mathematical equation (mental arithmetic) a high cognitive load can be elicited and observed. The cognitive load or demand that a task elicits depends on the information elements that are required to be held in the working memory. Working memory can process up to around seven information elements at the same time before no new information can be processed. This number of elements decreases when the information not only has to be remembered, but 
processed $[97,98,99]$. If an individual is resting, there are few elements of information required; that individual is not processing anything new. If the individual is moving their arm (motor movement) to grasp an object there is more information to process, by seeing where the object is, grabbing it, judging the weight, and moving it. These are all elements of information that need to be processed, even if we are not aware of the processing itself. These information elements lead to the brain exhibiting a higher cognitive load. If an individual is undertaking a mental arithmetic task they may have to process new information such as an unexpected number, whilst relying on long-term memory of theory related to the task. More information elements are added to the task, thus increasing the cognitive load. The cognitive load reflects the complexity of the task, and the complexity of the task can be used to elicit cognitive load. Having prior knowledge of a task lowers the cognitive load imposed by that task. To compare, humans use their limbs for motor tasks from early childhood, whereas processing new concepts such as mathematics is not expected from such a young age, or as frequently. These elements lead to cognitive load increasing along with task complexity; rest results in low cognitive load, mental arithmetic tasks elicit a high cognitive load, while motor tasks fall between these high and low cognitive load categories [97, 98, 99].

\subsubsection{EEG as a measure of Cognitive Load}

Certain elements of EEG can be used to assess cognitive load, otherwise referred to as cognitive demand. Like any electrical signal, EEG is a periodic signal composed of amplitude and frequency. The amplitude changes during tasks, but the frequency indicates the task being undertaken [100]. Essentially, the higher the cognitive load, 
the higher the frequency of the EEG signal [101]. EEG is a physiological index that can serve as a continuous measure of cognitive load, while detecting subtle fluctuations in instantaneous load. Functional magnetic resonance imaging (fMRI), which detects change in blood flow of the brain could also be used. However, compared to fMRI (mm range), EEG has low spatial resolution (cm range), making it more difficult to draw precise inference of brain location [101]. This should not affect assessment of cognitive load, as the brain location does not need to be precise for the proposed method. A central advantage of EEG over behavioural indices and other neuroimaging methods lies in its high temporal resolution, which also allows for the assessment of the time-course of cognitive load during execution of the learning task [102]. EEG is advantageous over verbalized tests as the brain wave rhythms reflect the changes in information processing, even if the participant is unaware of these changes or unable to reflect on them $[97,98,99]$.

One of the earliest examples of EEG being used to assess cognitive load was carried out by Penfield and Jasper (1954) by measuring the EEG output of Albert Einstein. Einstein showed continuous alpha rhythm while conducting complex mathematical operations, although they were quite straightforward by his standard. A sudden drop in alpha waves was detected when Einstein found a mistake in a calculation he had made the day before [33]. This assessment did lack experimental value in terms of only having one participant, but it did give a fascinating insight into a very famous professional thinker and gave insight to how EEG can detect changes in thought process. This investigation was just one element of Wilder Jasper's extensive work in neurophysiology. Jasper and Penfield (1943) showed how EEG can be used to detect changes in electrical brain activity indicative of epilepsy, caused by damage to brain 
tissue [103]. At this time, treatment for seizures was surgery, but this method allowed for detection and localization of the damage. This enabled immediate intervention without the risk of exploratory surgery. This research shows the value of EEG as a tool to understand and treat neurological conditions. There are certain patterns in research that show the validity of using EEG in terms of SMR, to assess cognitive function, as well as directing future methods. Some of these are shown in table 2.1.

\begin{tabular}{||cccc||}
\hline Brainwave & Observation & Cause & Citation \\
\hline \hline delta & More prominent in temporal region & Visual attention & {$[104]$} \\
\hline delta & Power increases & Arithmetic tasks & {$[105]$} \\
\hline theta & Increase & Episodic and working memory & {$[106]$} \\
\hline theta & Increase in magnitude & Difficulty of task & {$[101]$} \\
\hline theta & Band power decreases & Encoding new information & {$[101]$} \\
\hline theta & Increase & Higher working memory & {$[101]$} \\
\hline alpha & Suppressed & Increasing cognitive load & {$[107]$} \\
\hline alpha & Band power decreases & Encoding new information & {$[101]$} \\
\hline alpha & Increased band power in & Increased mental workload & {$[104]$} \\
& temporal and parietal regions & & \\
\hline alpha (high) & Increase & Semantic information processing & {$[106]$} \\
\hline beta (low) & Increase & Higher working memory & {$[101]$} \\
\hline beta & Increased band power in temporal, & Increased mental workload & {$[104]$} \\
& occipital and fronto-central areas & & \\
\hline
\end{tabular}

Table 2.1: Observed effects on specific brainwaves and the cause. 


\subsection{Audification}

We propose that EEG can be audified to enable detection of differences in the signal by novice users. Audification is the process of converting an electrical signal to an auditory signal. This can be done directly by using the electrical signal to drive a speaker. The key to successful audification is successful categorization of the information being conveyed [108].

Scientific data analysis and communication is usually conducted through visual mediums such as databases and graphical interfaces [29]. This can be counterintuitive and complex, especially with large amounts of data. The support for audification is grounded in various applications that have shown audification can be more effective at detecting small changes in data [30]. Seismic data, for example, can be audified and the resulting sound produced is similar to natural environmental sounds, allowing for easily detectable differences. This is because sounds transmitted through air (acoustic waves) have similar physics to seismic vibrations transmitted through the earth (elastic waves) [109].

Medical students are taught to rely on their hearing when understanding the human body. The best example of this is the use of a stethoscope to listen to tissues rubbing in the lungs, gases bubbling in the intestines, and blood pumping through the veins [110]. Medical students perform better in a simulated operation when the variables related to the patient's condition are displayed with sound compared to graphical displays. This performance is better with sounds alone, rather than a combination of sounds and graphs [30]. By mapping image texture to sound, unhealthy regions of a brain scan can be identified when hovering a mouse cursor over them [111]. This data supports that humans are better at multitasking and deciphering separate 
variables when transmitted with sound, as compared to visual mediums.

Human hearing can filter out the sounds that are not useful. For example if someone is trying to navigate a busy town, they can focus on the continuous line of traffic over the commotion of people talking to each other [112]. The human ear has access to a single pressure wave which is a combination of all individual pressure waves. This allows for easy detection in changes of time and order, when they are encompassed in one audible stream [113].

Audification of EEG for detecting abnormal brain activity has been shown to be feasible but has not been researched extensively. For example, audification has been used for neonatal seizure detection by using a probabilistic method to assess the likelihood of a seizure [114]. Human hearing is used to accurately assess spatial and temporal evolution of the frequency characteristics, by translating these aspects of the original signal into change in pitch and tones in the audio signal. This method can be of benefit to clinicians and help increase the detection rate of neonatal seizures.

A similar method used the placement of two electrodes to measure and audify EEG [115] by compressing the data into a higher sample rate. EEG data were recorded from patients with temporal lobe epilepsy and converted to an audio file using opensource software. Participants underwent minimal training - between 2 and 17 hours. They were then able to detect seizures using the audified signals alone, when compared to healthy signals. An improvement on false detections was also observed when compared to other methods of detecting seizures.

An earlier study showed that auditory representations of biological signals can complement visual representations [116]. Data was taken from sleep clinic patients and compressed, allowing for a whole night of sleep to be observed in a few minutes 
of audio data. The researchers developed a simple method of converting EDF files, which is a very common format of EEG data, into WAV audio file format. This helped assess the relationship between different phenomena during sleep, whilst showing how the different wave bands of SMR behave. This method allowed the measure and distinction of SMR in specific bands during different types of brain activity, mainly REM and non-REM sleep.

\subsubsection{Parameter Mapping}

While audification converts the direct signal to an auditory range, a more common approach is for the data dimensions to be mapped onto an auditory parameter, known as sonification which can include duration, loudness, position, frequency and brightness. The key distinction is that audification encompasses a signal that is comprised of the original data, with minimal interference or editing. Sonification is an integration or translation of the data into another medium, compiled of new and synthetic variables. Different variables can be mapped to different parameters at the same time to produce a complex sound. Existing software allows for straightforward mappings of many auditory parameters. The advantage of this method is that multidimensional sounds can be produced to translate more complex data; however, this can produce unpleasant sounds [114]. Sonification comes with its own potential pitfalls, such as the sound itself causing distraction, fatigue from unpleasant sounds, and comprehensibility of sounds differing significantly between users [117].

It is possible to detect ongoing seizures by sonifying EEG data [118]. Early intervention of seizures is crucial to avoid prolonged damage to the brain. Two recent 
studies have shown that untrained individuals can successfully identify the sonification output related to seizures, when compared to the sonification of healthy brains $[119,71]$. Detecting seizures through visual techniques, such as reading EEG outputs, can take hours to complete and requires professional expertise. The sonification method achieved a ninety-five percent successful identification rate with nonexperts, compared to a fifty percent success rate of reading visual EEG outputs.

Audification differs from sonification in the way that the signal is converted to the auditory range. Sonification depends on a mapping of sound chosen by the researcher. The method of audification relies on changing just the frequency of electrical signal an the audible range. Audification is beneficial for detecting these fluctuations because human hearing has great acuity when comparing slightly mismatching sounds [120]. However, this is not a straightforward process as the power spectrum of EEG decays much faster than the audio signals we are more commonly exposed to, such as music. Music is developed and tuned specifically for our ears, whereas natural electrical signals, such as the ones exhibited by one's brain, are not produced for the audible spectrum [121].

Sonification can involve the use of machine learning, which is advantageous in terms of repeatability and automation [122]. However, this may lead to inadvertently cutting out important data. Audification is chosen for this research because the brain is better at processing EEG data than an artificial intelligence system would be. The aim is to audify an electrical signal with minimal manipulation. This allows for a realistic representation of the source data without sacrificing elements that could be deemed irrelevant, but may lead to unexpected findings. 
The next section of this thesis describes the work undertaken in the initial design of a device to detect EEG and audify the signal. It discusses a hardware approach to audification and leads to a software method. The software method to audify signals that already been collected was necessary in light of the COVID-19 pandemic when research with human participants was halted. The chapter ends with a description of the different signals developed for audified presentation to novice observers at varying levels of cognitive load. 


\section{Chapter 3}

\section{Methods}

\subsection{Preliminary Device Design}

The first step in designing a device for the audification of EEG post-concussion is to understand the user needs and specifications. The idea was to develop a standalone device that could measure EEG with surface electrodes and play the audified version of the EEG signal through a speaker or earphones. A design process was followed as discussed below starting with the identification of the user group and user requirements.

\subsubsection{Identifying Target User Group}

The target user group was identified as individuals who are the first to assess an individual who suffers a brain injury. In everyday life, this could be a paramedic. If someone calls emergency services after losing consciousness or bleeding, the paramedic could assess the likelihood of a concussion before arriving at the hospital. This would save precious time for the doctors in the emergency room.

In a sports setting, this device could be used by a team medic when a player has 
suffered a suspected head injury. When a player is injured, quick diagnosis is key to

judging the need for further treatment. Access to objective assessment is expensive and rare, so sports teams and players rely on subjective assessment. The issue with this approach is that it can be influenced or interfered with by a coach, or the player themselves. This device would facilitate an objective assessment as soon as possible, allowing a medical professional to make a judgement call about investigating further. Having a warning system in place to assess the of risk and likelihood of concussion would help reduce the prevalence of mis-diagnosis, repeated injuries, and long-term effects.

\subsubsection{Design Criteria}

Here we outline the design criteria for an intended prototype of the device:

- Self-contained, it should not rely on external hardware or software to operate. This will facilitate plug-and-play assessment, causing minimal confusion and room for error.

- Compatible with simple and mass-produced electrodes that can be plugged into the device and can be easily replaced.

- Use minimal number of electrodes so that the system is easier to set up and use. The required placement of these electrodes must be achievable without in-depth understanding of the structure and function of the brain.

- Output system that allows for direct listening, even in noisy and busy environments.

- The circuitry of this device must be insulated against noise, to avoid disturbances from external sources. 
- The circuitry must encompass minimal connections that are resilient to movement and will not loosen easily. This will help reduce noise within the circuit.

- The circuitry will be housed in a rigid material that is not prone to fracturing, allowing for portability.

These criteria were determined by the researchers to focus on the hardware used for data collection. Future iterations would involve the target user group in a codesign effort to ensure that usability is embedded in the design process from early on.

\subsubsection{Device Design}

The device was to include a method to collect EEG signals from two different electrode locations, using a differential operational amplifier to eradicate noise from the raw EEG signal. A differential amplifier is an effective method for reducing noise on input, and requires a minimum of two input signals. The EEG signal would then be amplified using an isolation amplifier that is specifically designed for use with biological signals. This would amplify the EEG signal to a voltage capable of driving a speaker. The hardware design allows the EEG signal to be combined with another signal generated by the device. This would allow merging of EEG signals with an audible signal to result in auditory signals. The research later focused on software manipulation, allowing us to focus on a single electrode location and filter out the noise using Matlab. The design aspect of combination was later removed, and replaced with compression using a software approach. 


\section{Components and Construction}

The circuit design construction can be found below matched by number with the schematic in fig. 3.1 to fig. 3.6. The schematic as a whole can be seen in fig. 3.7. The PCB layout of the design is shown in fig. 3.8.

1. Two EEG inputs are connected to the AD210, with a differential operation amplifier connected at input. The input to the AD210 includes a potentiometer, allowing for immediate change of gain for the EEG signal. The strength of the EEG signal will vary slightly between connections, so a variable gain is important.

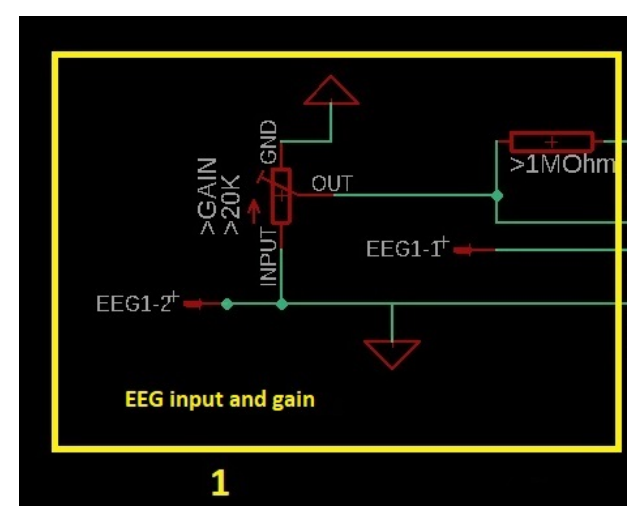

Figure 3.1: A potentiometer is a type of resistor that allows for real time change of resistance. Set up in this way, with another standard resistor, creates a differential amplifier. A differential amplifier will remove noise from both input signals and amplify them, based on a controlled gain.

2. The AD210 is an isolation amplifier specifically designed for biological signals that reduces the noise that may saturate the EEG input. The AD210 requires a dedicated power supply of 15 Volts. Ideally this would be a set-up that includes combining conventional $9 \mathrm{~V}$ batteries, rather than mains supply as mains power 
would contribute significantly to noise.

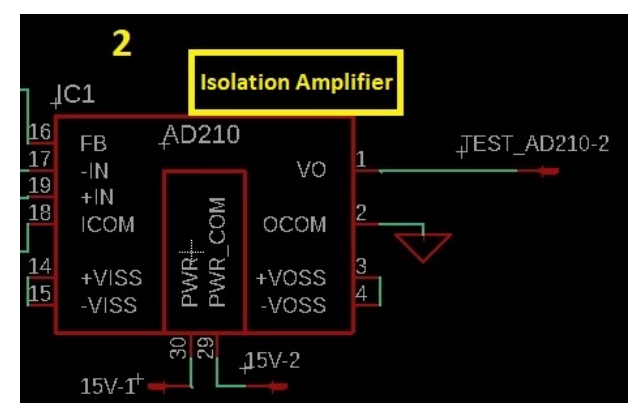

Figure 3.2: The AD210 isolation amplifier uses a chosen gain to amplify the signal to the desired range, whilst removing noise.

3. The circuit design allows for testing post-amplification and at every section on the circuit. These test bridges would be removed when moving to manufacture the full system.

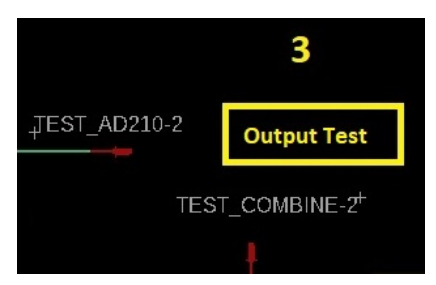

Figure 3.3: These test points allow for troubleshooting the initial prototype, and can then be connected to the inputs seen in fig. 3.6.

4. An Arduino was used to produce a digital signal connected to a digital to analog converter (DAC). This produces an analog carrier signal that can be combined with the EEG signal. 


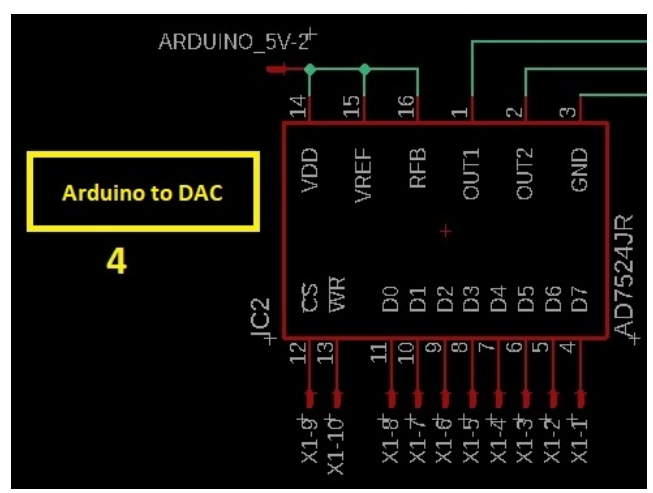

Figure 3.4: An Arduino is programmed from a computer and powered by a dedicated battery.

5. Potentiometers are connected to the output of the DAC to allow for amplitude adjustment to allow the carrier signal to be produced at the optimal magnitude for the headphone output. This amplification can also be changed to evaluate the effect it has on the audification process.

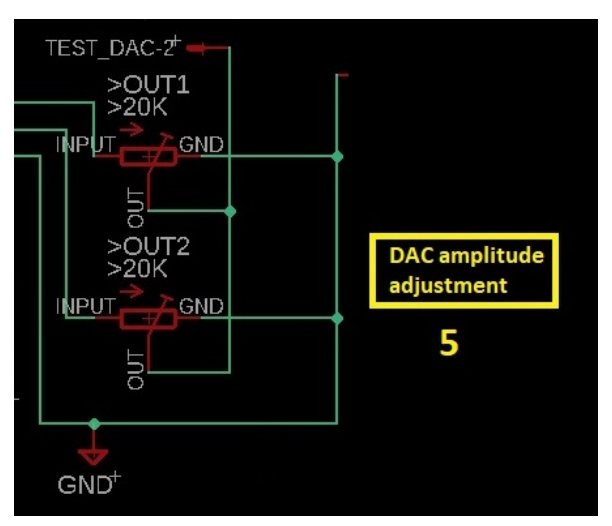

Figure 3.5: A combination of potentiometers allows for testing of the generated signal before combing with the EEG signals.

6. Both signals are then combined and passed through the earphone output, to enable the listener to interpret the signals from the audification process of the combined EEG signal and carrier signal. 


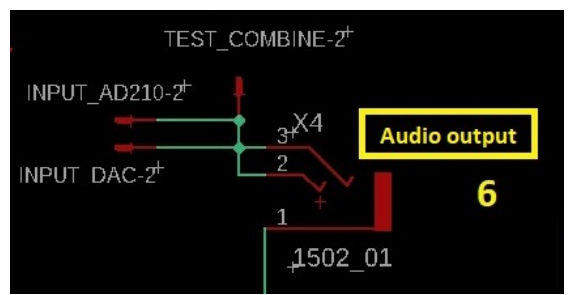

Figure 3.6: Here an earphone output is used, for direct listening to the combined signals in one audio signal.

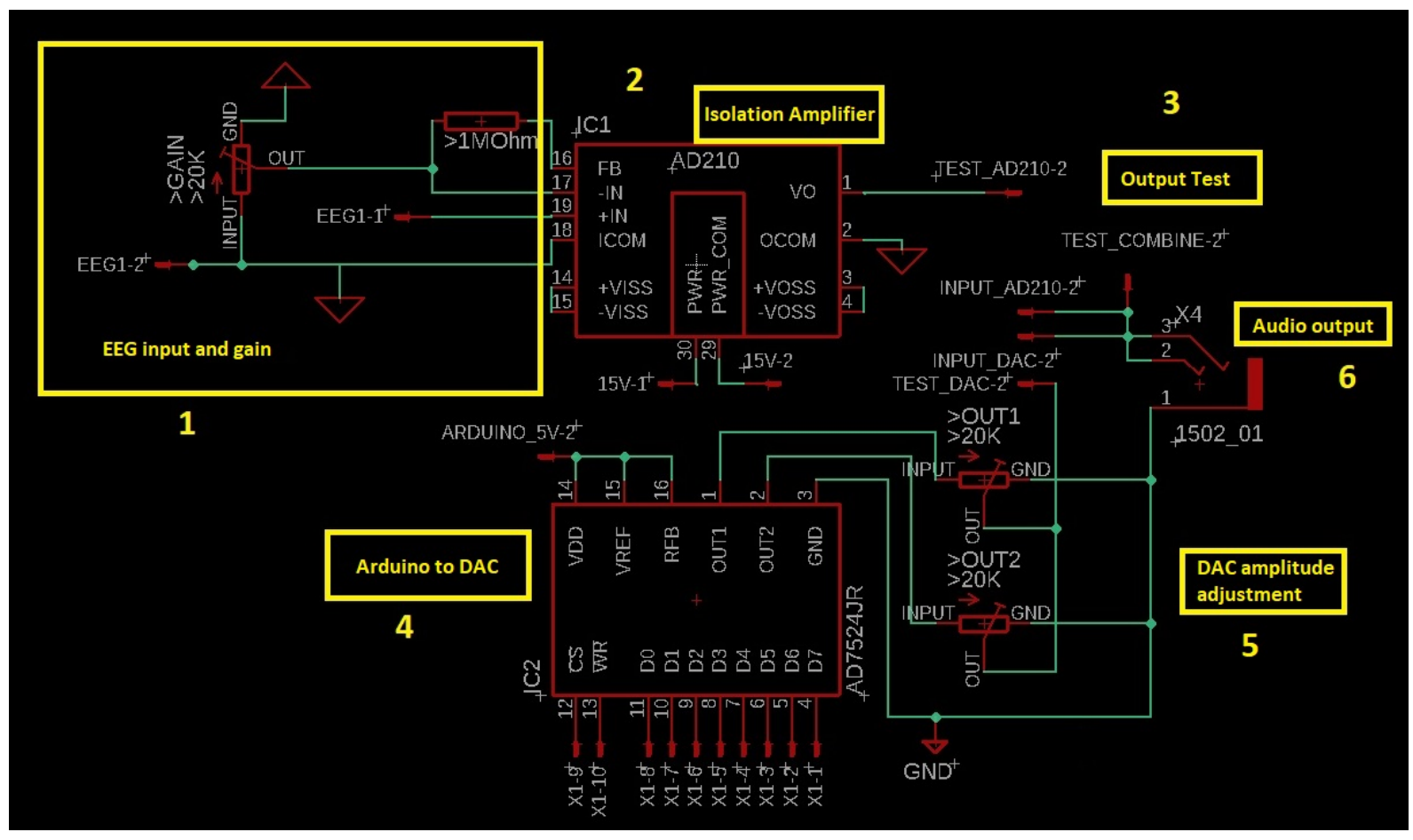

Figure 3.7: Circuit schematic for design of the prospective device.

EEG, even when amplified, is comprised of frequencies between 0 and $100 \mathrm{~Hz}$. Human hearing can only detect sounds of roughly $20 \mathrm{~Hz}$ and above, so an audified signal of EEG would not be fully detectable by the human ear. The carrier signal from the Arduino is fed through a digital to analog converter to produce an alternating current signal that is powerful enough to drive a speaker. The theory is that the 
carrier signal would combine with the EEG signal for a unique signal comprised of both the carrier and EEG signals.

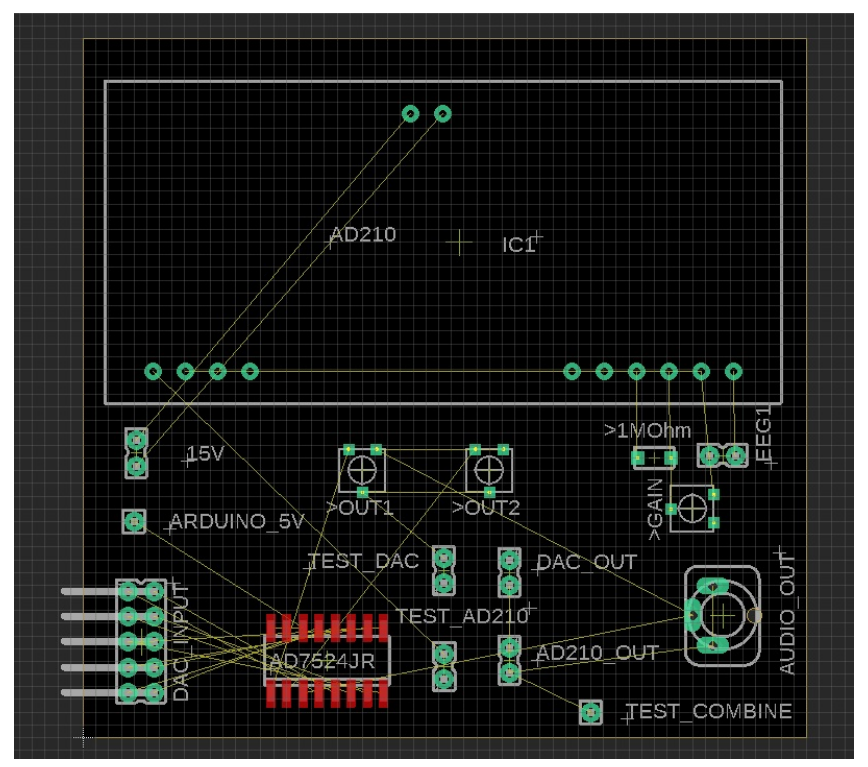

Figure 3.8: Circuit layout for design of the prospective device.

Preliminary testing was conducted using a breadboard circuit and basic components. Electrodes were attached to the researcher's head. The signal was amplified and connected to a speaker. Despite changing the amplification, no detectable difference was observed. The artifact noise, as a result of using a breadboard, was significantly greater than the brain signal, causing lack of detectable differences. Further testing was conducted using a protoboard, soldering the parts in place, to determine whether more secure and dedicated wiring would reduce the impact of noise. This did not appear to make any notable difference to the noise saturation. The EEG signal itself was too weak, in comparison, and was saturated by external noise. Future work would involve designing a circuit board that isolates the EEG signal from external noise, ensuring that amplification of EEG signal allowed for detectable differences. 
The method of collecting and measuring EEG using circuitry proposed was unreliable. The plan was to develop a reliable and accurate circuit, and then fine tune the EEG collection process. However, the inability to conduct in-person testing during the pandemic led to the use of an open-source dataset with software compression as a means to audify the signal.

\subsection{Audifying EEG Signals}

When COVID-19 restrictions reduced access to the lab, it became apparent that building a device would not allow for the best outcome since testing with participants would not be possible. This led to an experimental change in which access to a database of previously collected EEG signals from tasks of varying cognitive load [3] were audified and provided to novice listeners to evaluate ability to detect changes in that cognitive load. The same method of detection could be used in the future to test signals collected within our own laboratory setting.

The purpose of this chapter is to explain the process of audifying previously collected data from an online open-source database which was then provided in sound files to novice listeners in the following chapter.

\subsubsection{Preparation of Data}

The dataset used for this study consisted of EEG measurements taken during rest, mental arithmetic, and motor imagery tasks [3], where motor imagery refers to the task of imagining the movement of a limb without carrying out the movement itself. The data was collected according to the protocol shown in fig. 3.9. From this dataset, 
four types of audio files were generated, one associated with each: mental arithmetic task, rest portion of this data, motor imagery task, and the rest portion of this data. EEG data of 29 participants were collected and made available through an online database. Each participant had been tested with 30 active electrodes and data were collected with an EEG headset (Brain Products GmbH, Gilching, Germany). The placement of electrodes was in accordance with the international 10-20 system [123]. The data encompassed EEG activity from two tasks assigned to each participant. The first task was a visual mental arithmetic task, involving equations being displayed on a screen in front of the participant. The second task involved the participant imagining the opening and closing of their hands, as if they were grabbing a ball. This action is known as kinaesthetic motor imagery.

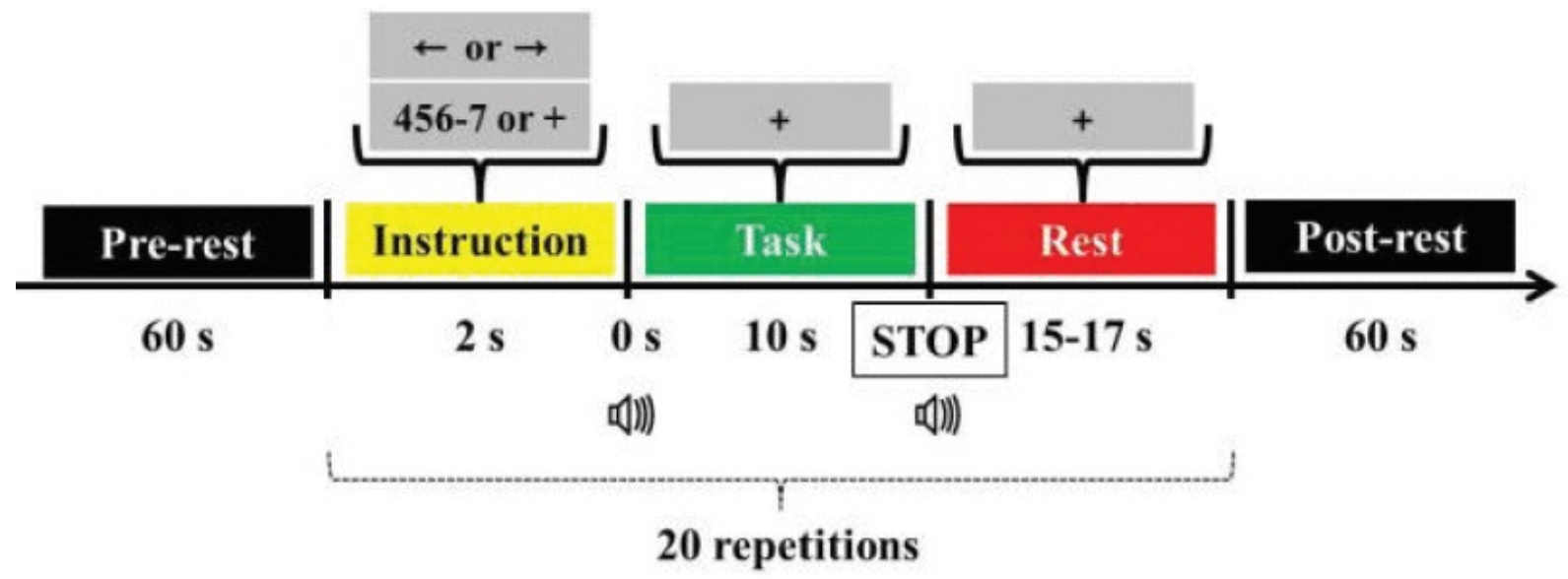

Figure 3.9: Visual representation of the task and rest data, as it was recorded for the open-source dataset [3].

\subsubsection{Matlab Processing}

Fig. 3.9 demonstrates the method of collecting the data provided through the online dataset. Matlab (TheMathWorks, Inc., Natick, MA, USA) was used to extract data 
from the dataset in terms of task data and rest data. Aligning with the times identified in fig. 3.9, data extraction was undertaken to separate specific tasks and rest sections. The methods outlined in Shin et al (2017) were very detailed and were integral to successful extraction and use of the data. Based on the described methodology, the specific time stamp of a data cell was measured which allowed for the separation of data for each task. The dataset was organized so that different files contained the data from each task (see fig. 3.10). Data were extracted from these files in two steps:

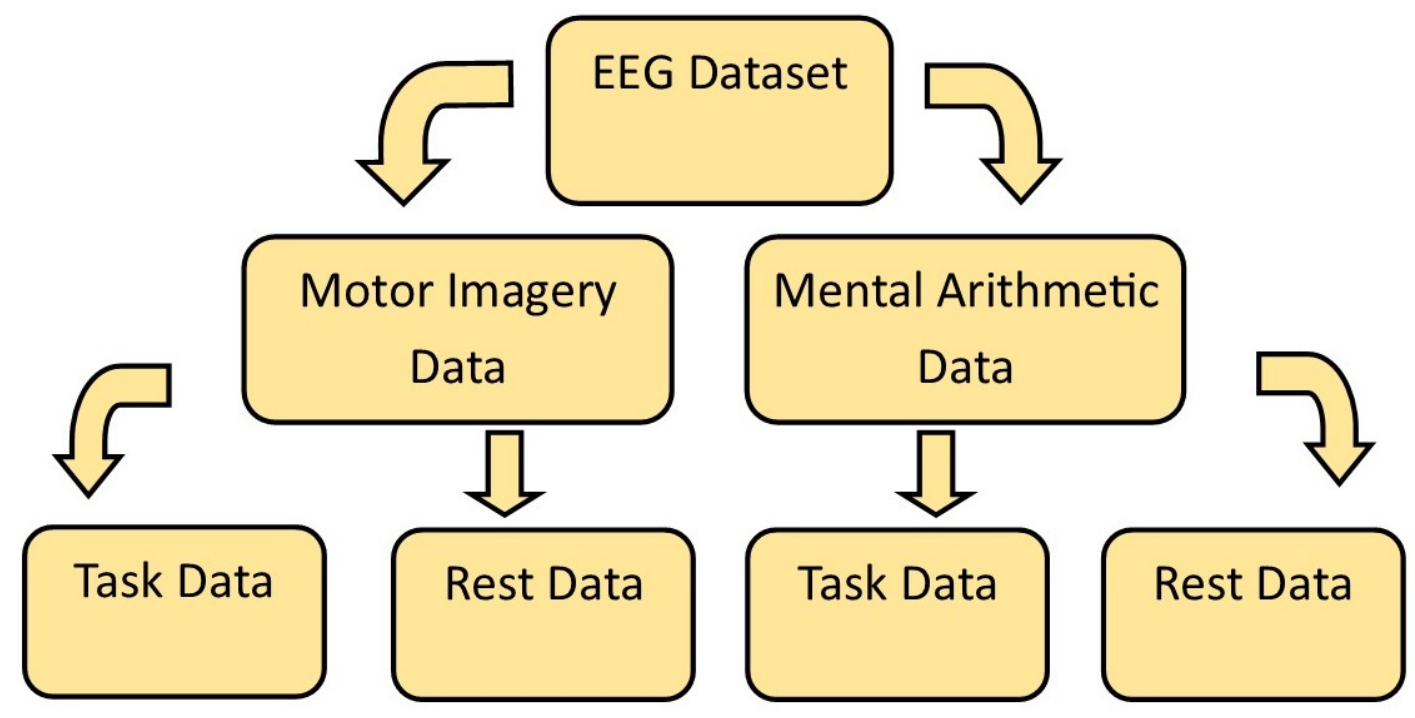

Figure 3.10: Demonstrating how the data were extracted from the dataset for use in Matlab.

1. Extract the data relevant to the task itself.

2. Extract the data relevant to the rest section.

The next step was to save the relevant data to a file for processing. Four files of EEG data were created. There were two tasks, but each task required two files: 
1. Motor imagery task data.

2. Motor rest data.

3. Mental arithmetic task data.

4. Mental rest data.

\subsubsection{Amplification}

Fig. 3.11, fig. 3.12, fig. 3.13, and fig. 3.14 show the time series plots of the amplified EEG outputs of different tasks. The examples of data used in this thesis are all from participant one of twenty-nine that were available from the dataset. The data collected from the dataset was in the range of hundreds of microvolts. For these time series plots a gain of 20,000 was required to get the signal to an appropriate level of voltage for driving a speaker or earphones, which require between two and five Volts. This amplitude did not affect the simulation and software audification process, but was important to show the proof of concept of manipulating the signal in a realistic manner. Time series plots of the data pre-amplification can be seen in Appendix C. 


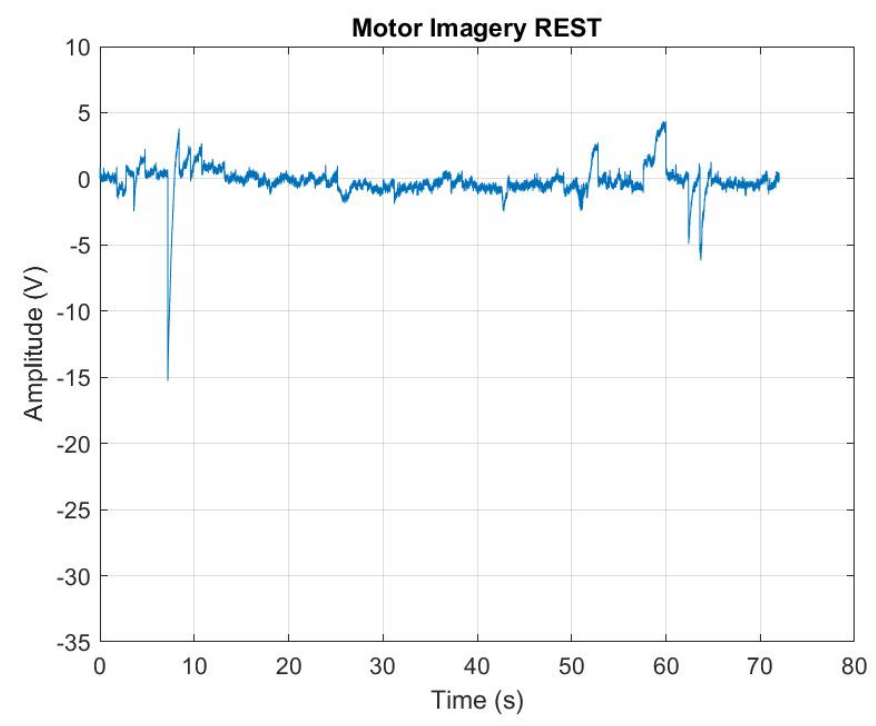

Figure 3.11: Amplified EEG data relevant to the rest portion of the motor imagery task.

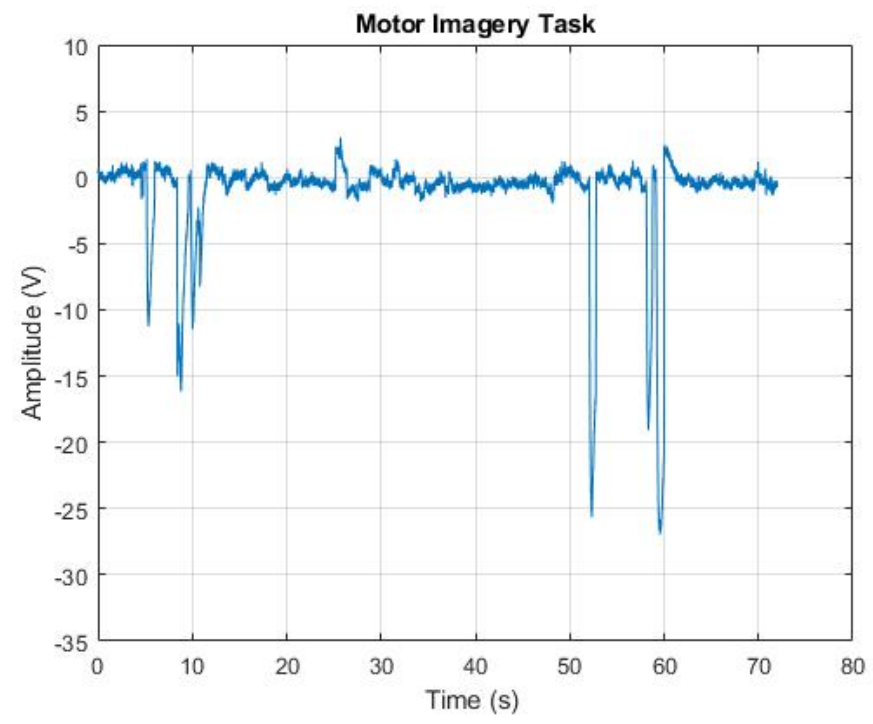

Figure 3.12: Amplified EEG data relevant to the motor imagery task. 


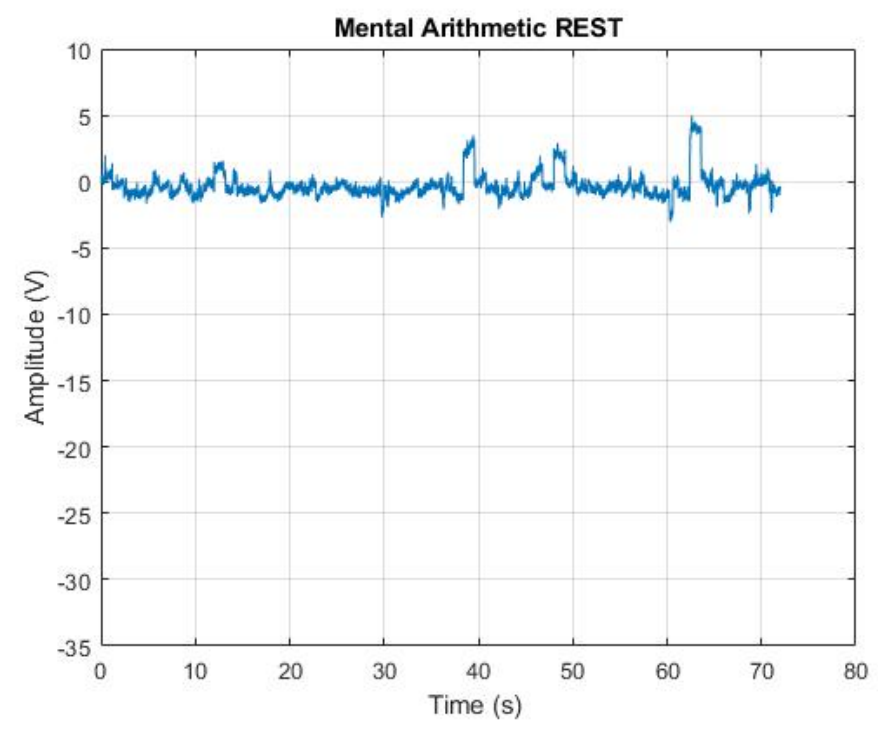

Figure 3.13: Amplified EEG data relevant to the rest portion of the mental arithmetic task.

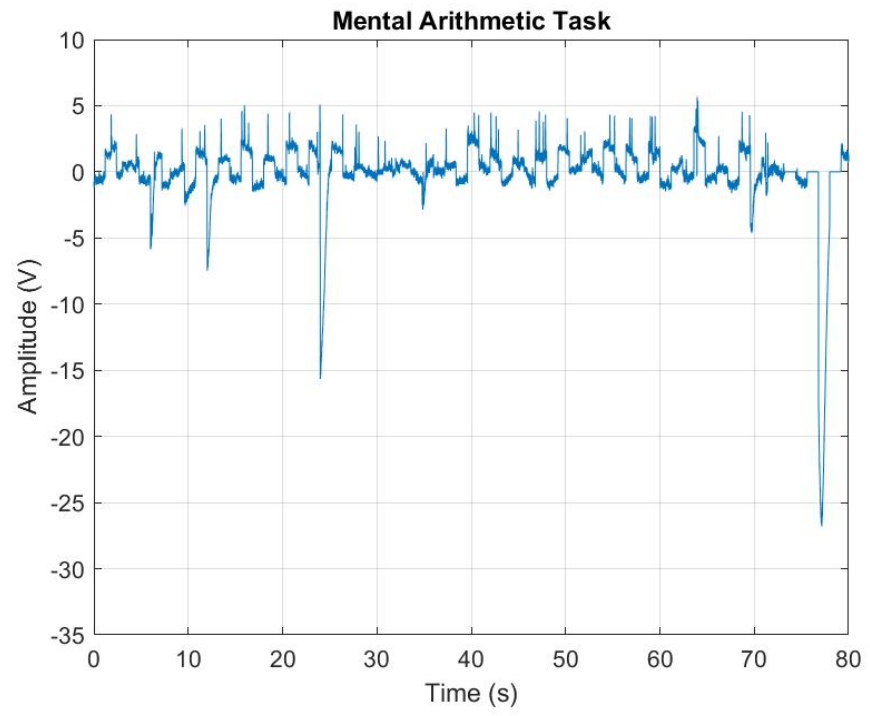

Figure 3.14: Amplified EEG data relevant to the mental arithmetic task. This data is observed over a slightly longer time because these sections of the data set were recorded over a slightly longer time frame.

The two rest phases show little difference in amplitude; however, both cognitive 
tasks show more high and low peaks compared to rest. Mental arithmetic produces data that embodies more peaks and troughs, compared to other data. The data changes more rapidly in the same amount of time and exhibits a higher average amplitude. These two factors may be key to audification:

1. Higher average amplitude (compared to average of other tasks/data).

2. Variable data, rapidly changing between higher and lower amplitude.

\subsubsection{Filtering and Compression}

Compression can be viewed as a generic and technical term. In data manipulation it is commonly associated with reducing the size of a file, by removing some elements of the data that are unnecessary. In this research, we use the term compression to refer to a compression in the length of time over which a sample is recorded. This allows us to view all the data in a shorter amount of time and audify the data in a frequency that is detectable by the human ear. No data is removed in this process rather, it is just displayed in a different time scale. The sampling frequency of the original data was $1000 \mathrm{~Hz}$, until we compressed it by a factor of five, leading to a new sampling frequency of $5000 \mathrm{~Hz}$. For example, any data originally recorded at a frequency of $10 \mathrm{~Hz}$, could now be observed at a frequency of $50 \mathrm{~Hz}$, allowing it to be within the range of human hearing. Fig 3.15 shows an example of compressing a $1 \mathrm{~Hz}$ signal by a factor of five. The lower frequency signals are chosen purely as a visual aid. 

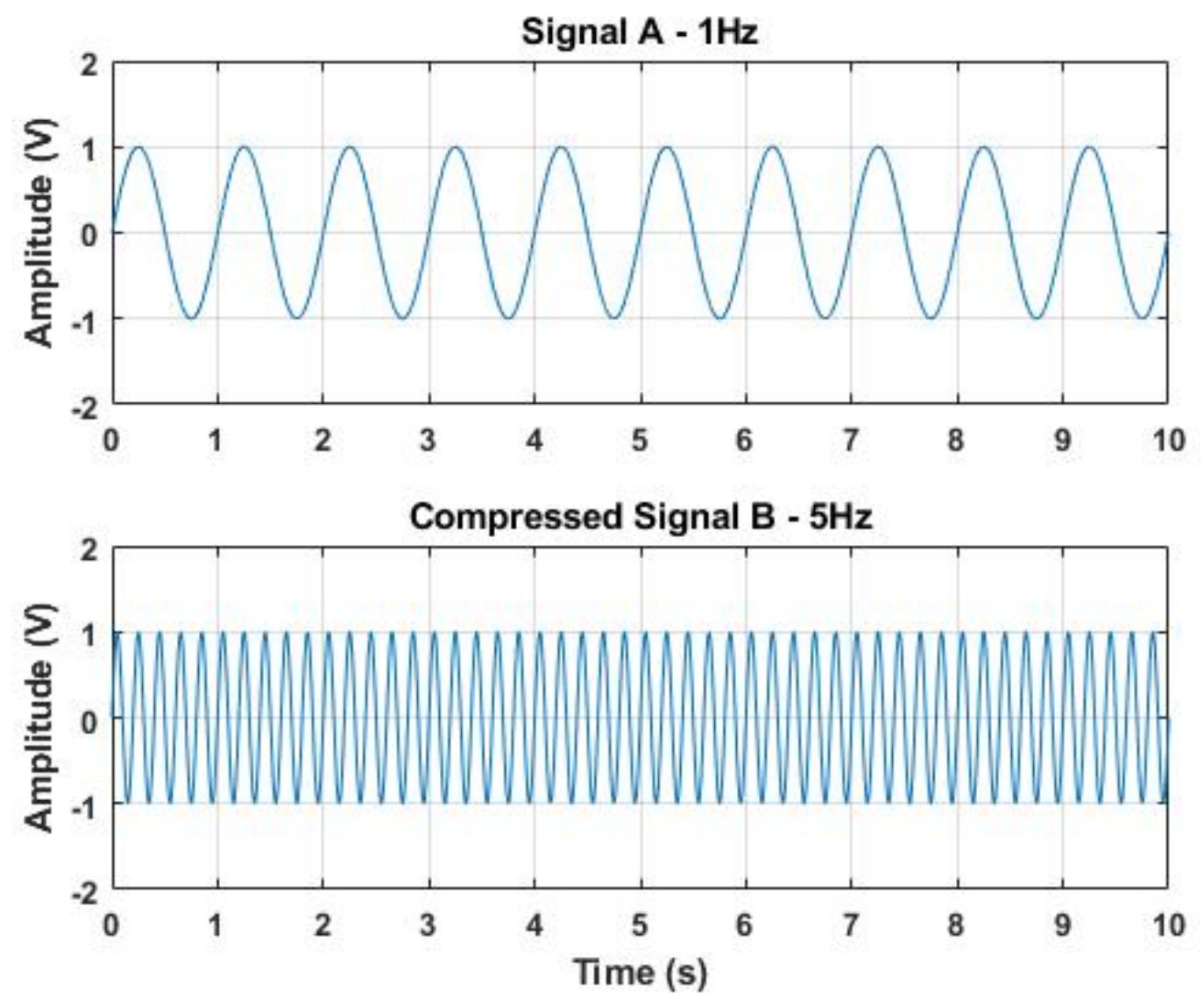

Figure 3.15: Showing compression of an arbitrary signal.

Extraction of the different wave types (delta, theta, alpha, beta, and gamma) was important as each represents differing degrees of cognitive load. Beta waves are common in various types of behaviour that require little cognitive processing, whereas gamma are only observed during tasks that are cognitively demanding. Beta is also observed at a higher amplitude so gamma can be saturated or overshadowed by the existence of beta, when observed in the same medium [124]. When audifying it is important to filter the gamma waves separately to allow for detection of small changes in gamma, that would otherwise be saturated by other wave types. 
Matlab was used to simulate a fourth order Butterworth bandpass filter to split the data into separate bandwidths defined by the SMR waves. Plots were used to visualize the behaviour of each type of brain wave during different task - fig. 3.16, and fig. 3.17 .

To be audified, the signal needed to be modified to provide a sampling frequency in the order of $5000 \mathrm{~Hz} .5000 \mathrm{~Hz}$ was determined to be the minimum sampling frequency for successful audification. This is supported by research using Fletcher-Munson curves stating that the human ear is most responsive to sounds between $500 \mathrm{~Hz}$ and $5000 \mathrm{~Hz}[125]$.

The auditory files were generated using Matlab, with five different filter bandwidths, associated with the different brain wave bandwidths (roughly) - delta (0-4Hz), theta $(4-8 \mathrm{~Hz})$, alpha $(8-12 \mathrm{~Hz})$, beta $(12-30 \mathrm{~Hz})$, and gamma $(30-100 \mathrm{~Hz})[64]$.

As seen in fig. 3.16, and fig. 3.17 no discernible differences were visible in the delta band wave, at least not in the representation. That can be attributed to delta mainly being present during deep sleep, an activity not recorded in this dataset $[65,1]$. From fig. 3.16, and fig. 3.17 it does appear that theta is the most prominent and therefore would be more effective for audification. Presence of the data and strength of the signal appears to decrease from theta to gamma, with gamma being the weakest signal apart from delta. The data shows that theta waves are present during any type of cognition, with increased presence in the mental task compared to the rest section. Higher cognitive load leads to increased presence of all types of brain waves, and some elements are consistent through, such as the peaks that are visible during 24 seconds and 77 seconds on fig 3.16. 

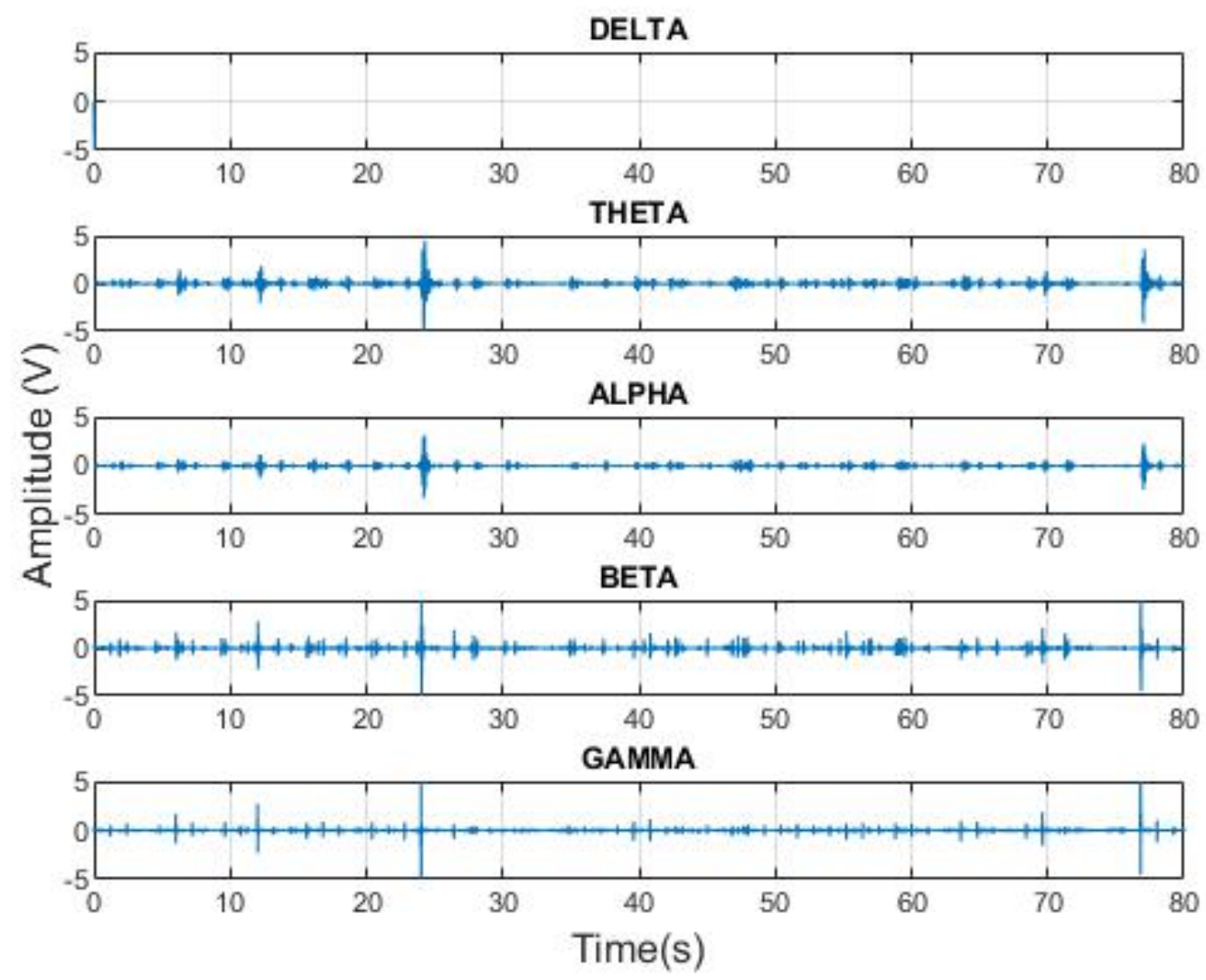

Figure 3.16: EEG data relevant to the mental arithmetic task filtered into brainwave bandwidths. 


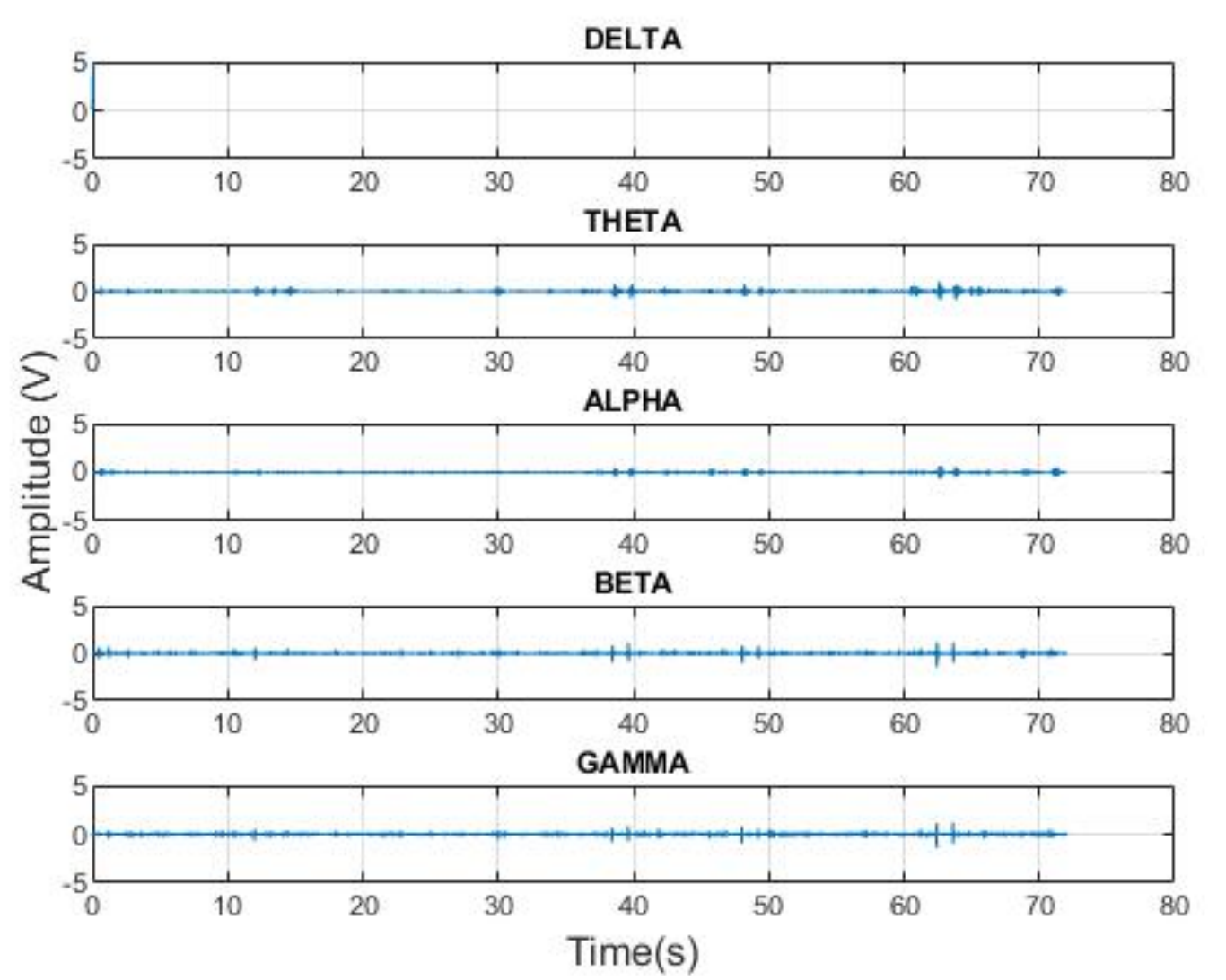

Figure 3.17: EEG data relevant to the mental arithmetic rest filtered into brainwave bandwidths.

\subsubsection{Audification of the online Dataset}

The individual signals of the EEG data were filtered and then compressed to a sampling frequency of $5000 \mathrm{~Hz}$, maximizing the data transmitted in the audible spectrum, whilst still having a sample of about ten seconds to allow for attentive listening to changes in the signal. The Matlab "audiowrite" function was used to save the signal as an audio file. This was done in WAV format. The audio file was saved with the normalized data at the compressed sampling frequency. 
Once the audio files were generated, they were trimmed. The original length of the audio file was fourteen seconds, but two seconds at the beginning and two seconds at the end of the file were removed. The purpose of this was to remove any data that may be reflective of an adjustment period or expectancy for the task to end. When a participant begins a task, they may take a second or two to adjust, meaning the beginning of the data stream isn't an accurate representation of the impact of the task. A participant may also stop paying attention toward the end of the task, meaning that end of the data is also not an accurate representation. Trimming resulted in a ten second audio file, consisting of the middle ten seconds of the original file.

Initial observations of data and decisions about audification were carried out by the researcher. With time constraints and pandemic related restrictions, it was not feasible to get further input from observers not involved in the research. To reduce experimenter bias, these audio files were listened to in a blind manner. It was important to determine that a difference was detectable after audification, as well as to clarify which files exhibited these differences, and compare for consistency. All the audio files were generated in a systematic manner. Four audio files were generated, one for each task - mental arithmetic task, rest between mental arithmetic tasks, motor imagery task, rest between motor imagery tasks. These four files were generated for each type of brainwave, for each participant of the dataset data. This resulted in twenty audio files for each electrode, per participant.

\subsubsection{Electrode Location}

Thirty different electrode test sites were used for the source dataset. However, for a device to be used in a timely manner, only one or two electrodes should be required. 
Audification of the signals from all test locations was performed and the researcher evaluated each location to identify the greatest discernible differences between cognitive load when a participant undertook a motor imagery or a mental arithmetic task. It was expected that both the mental arithmetic and motor imagery data would have an audible difference when compared to the rest data.

The focus for being able to identify differences in motor imagery and mental arithmetic was on the central and parietal lobes as these areas are associated with motor and mental processing, respectively [75]. It was ideal to focus on areas away from the frontal region, as recordings from the frontal lobes are more likely to contain eye twitch artifacts. This pilot testing by the researcher included audification of compressed gamma waves. This provided evidence that increased cognitive load was more prominent with mental arithmetic data in the parietal region of the brain. The research further focused on mental arithmetic data as high cognitive load appeared to be the only data that gave a reliable difference in terms of audification. Multiple scalp locations were tested to determine the location at which mental arithmetic was best measured.

The researcher assessed which scalp locations provided the most notable difference between audified data from the tasks. This was a very subjective approach to see which areas appeared to give an audible difference in data was shown to be unique during the time series plots. The researcher listened to all twenty audio files generated for one participant, for each electrode location. Tables were used to track participant, brainwave bandwidth, and scalp location. This was a time consuming task, motivated by Andres et al. (2012) who found the most reliable locations for 
detecting mental arithmetic activity are F3, F4, F8, P4 and Pz [126]. After exhaustive auditory assessment of these audified files, it was decided that the location of $\mathrm{Pz}$ facilitated consistent results across participants. The focus of all further research was on the $\mathbf{P z}$, in the centre of the parietal area of the scalp, as shown in fig. 3.18- which could allow for easy placement even if a user is not familiar with the international 10-20 system. The $\mathrm{Pz}$ location is also surrounded by other areas associated with mental processing [126], therefore accurate placement of the electrode is not integral to this process.

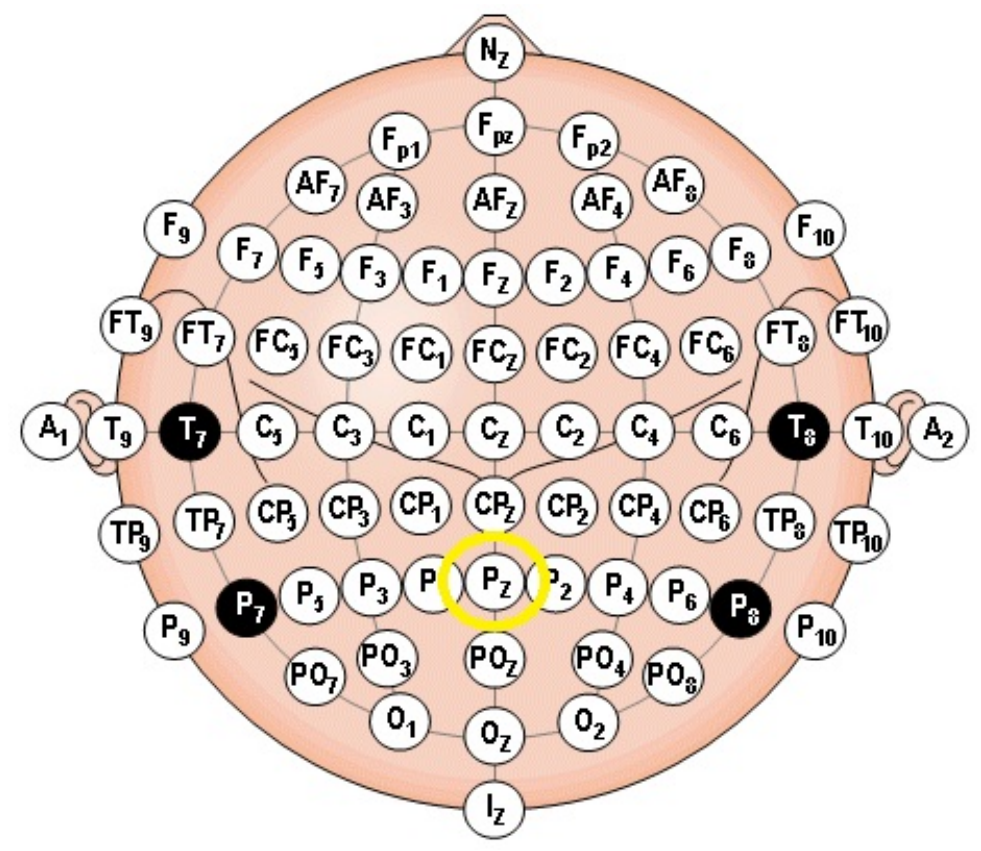

Figure 3.18: Highlighting the $\mathrm{Pz}$ location that is the focus of the work going forward [2].

One problem with collecting EEG from parietal regions is obstruction caused by hair. There is already significant impedance to the electrical signal of the brain activity, as this signal needs to travel through tissue and bone before reaching the scalp. The additional obstruction of hair can cause difficulties in achieving a strong 
and reliable connection when most people will have hair on that area of the scalp. The hair may get stuck in between the electrode and the scalp, significantly increasing the impedance of the connection, and making it more difficult to detect the small electrical signals observed in EEG. However, given the strength of the signal at this location, the ability to detect differences in behaviour, and the ease of location, this electrode site was deemed to ideal for the purpose of this research.

\subsubsection{Audified Output - Researcher Observation}

An audible difference among brainwaves was observed using the Matlab audification process. This was consistent across all the subjects in the dataset. It was expected that each SMR bandwidth would sound different because each is of a different frequency. The only discernible difference through audification was the prominence and volume of the unique attributes in the task data, when compared to rest data. This difference increased along with the frequency i.e., changes in sound were loudest in gamma, compared to theta, supporting the decision by the researcher to focus on the gamma region for the study. The audible difference between the beta and gamma waves is minimal but gamma appeared to be the clearest sounding, so the focus of the audification process was on the gamma region.

The main outcome of the audification process was that mental arithmetic data was more identifiable compared to other tasks. It was expected that mental arithmetic would result in a sound difference when compared to rest, because the time series plots show that it is of higher amplitude and higher frequency. It was was also expected that motor imagery data would create an audible difference, compared to rest. This was not observed by the researcher, and the motor imagery data appeared the quietest 
of all.

Audification of the theta, alpha and beta waves could be further investigated but would require much greater compression than gamma, requiring longer recording sessions. Gamma is useful with shorter data sets as it is comprised of the highest frequencies and minimal compression is required to make data within that bandwidth audible. To investigate the presence and use of the lower frequency brainwaves for audification, more data would need to be collected. It would be useful to analyze data collected over an extended period, as this would allow for compression idealized for each bandwidth so that audification can be assessed relative to each type of brainwave. However, we are seeking the minimize the time required to identify concussion, and taking longer readings limits the applicability for our purpose. For this study, only the gamma band audio files were used because these samples showed the most discernible difference during initial observations mentioned above.

After the researcher reviewed audified files from all participants, at various scalp locations, and for all brain wave bandwidths, the assessment focused on audified data in the gamma region and collected at the $\mathrm{Pz}$ location. After reviewing the gamma region audio files that were collected from $\mathrm{Pz}$ for each participant, the researcher observed that there was a clear distinction between the mental arithmetic data and all the other data. Mental arithmetic data appeared to create a sound that varied temporally, whereas the other data created a mostly monotonous continuous sound.

\subsubsection{Time Series Linked to Audification}

Fig. 3.19 and fig. 3.20 show the output for both versions of the rest data. From here we can see that they are very similar and do not vary much in terms of amplitude. 
They show similar peaks and troughs. These similarities translate to the audification process, and they sound almost identical. The anomaly in the motor rest data of low amplitude, circled in fig. 3.19, does not seem to affect the audio output of the data. This suggests that a one-off peak or supposed anomaly will not impact the audification process and that a trait in the data has to be consistent to impose an affect on the audified output.

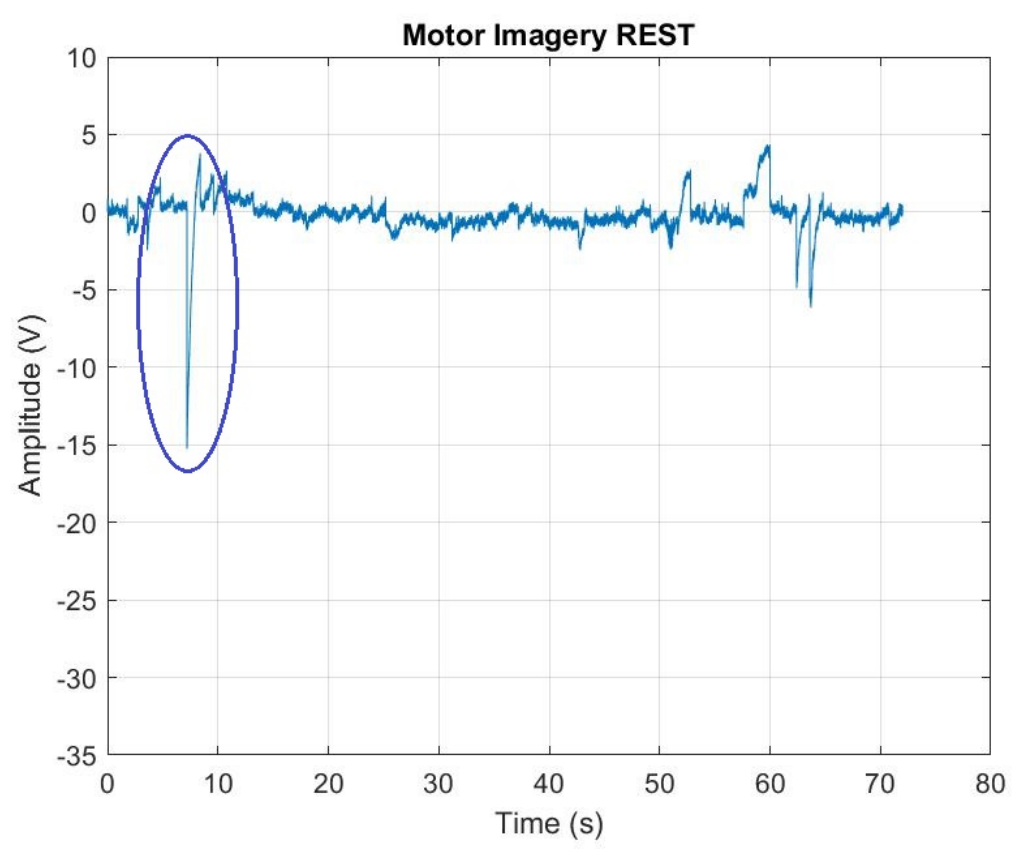

Figure 3.19: Amplitude of motor rest data with trough highlighted

Audified motor imagery data sounded similar to the rest data, in terms of being a constant hum with a few peaks in sound. The motor imagery plot has similar peaks so the lack of variance in sound makes sense; however, the overall volume of the motor data was lower than the others. This is because of the troughs exhibited in fig. 3.21 bring the average amplitude down, which relates directly to the volume of the sound file. Mental arithmetic created the most identifiable audio file, rather 


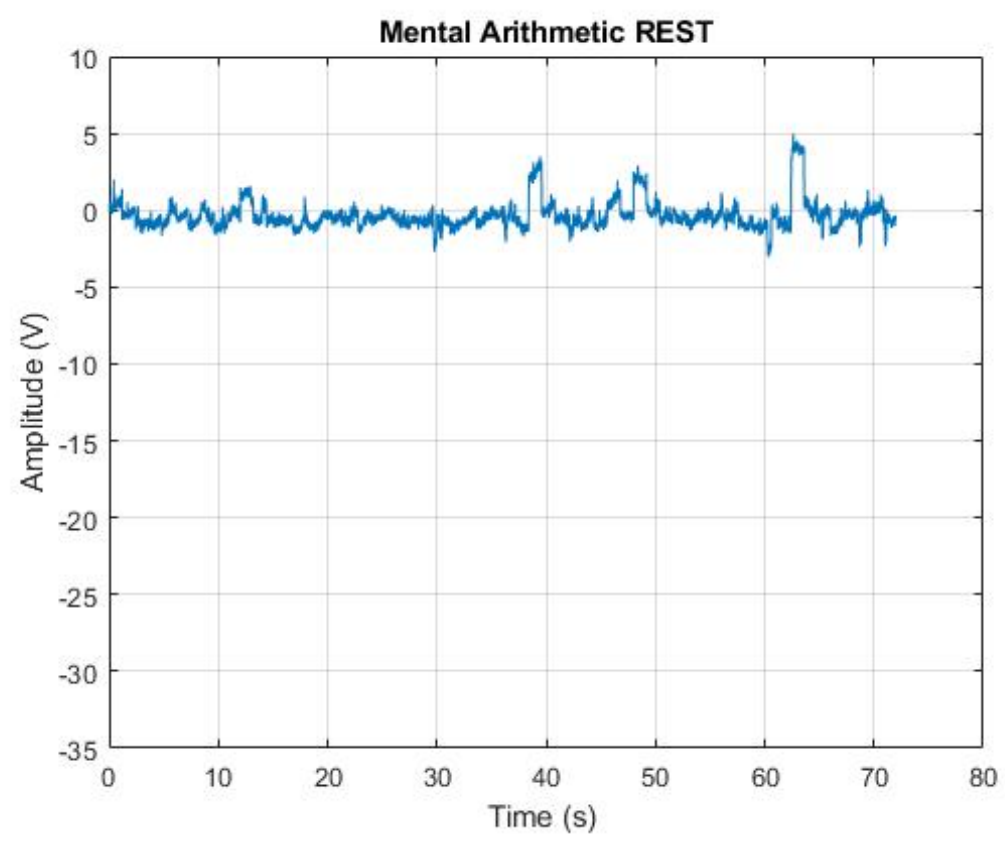

Figure 3.20: Amplitude of mental rest data

than a constant sound it created a signal that varied rapidly in terms of amplitude, analogous to a heartbeat. This is presumably due to the presence of multiple peaks, as seen in fig. 3.22. These peaks bring the average amplitude up, but this affect is balanced with the few large troughs. The key difference is the number of peaks which show the data is changing on a bigger scale and far more rapidly than data from the other tasks. This large and rapid oscillation of amplitude creates the oscillating sound on the audio file. This shows that even a relatively small difference in amplitude and oscillation can have a significant effect on the audifed signal. 


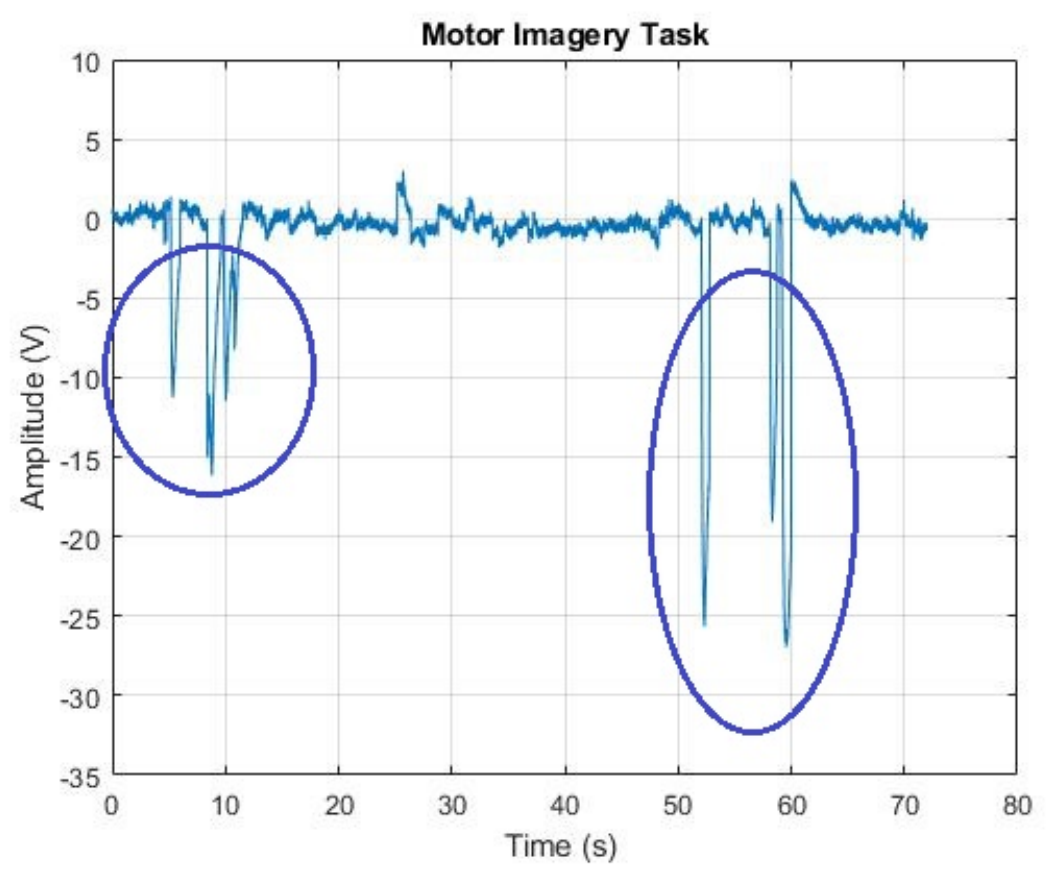

Figure 3.21: Amplitude of motor task data with troughs highlighted

It was expected that both motor imagery and mental arithmetic data would produce similar audified outputs because they both exhibit a higher cognitive load than rest. However, the plots and audification show that the cognitive demand exhibited by a mental arithmetic task is significantly higher than the demand of motor imagery. This is supported by cognitive theory in terms of processing information elements $[97,98,99]$ and also suggests that only a sufficiently high cognitive load will produce a difference when audified. 


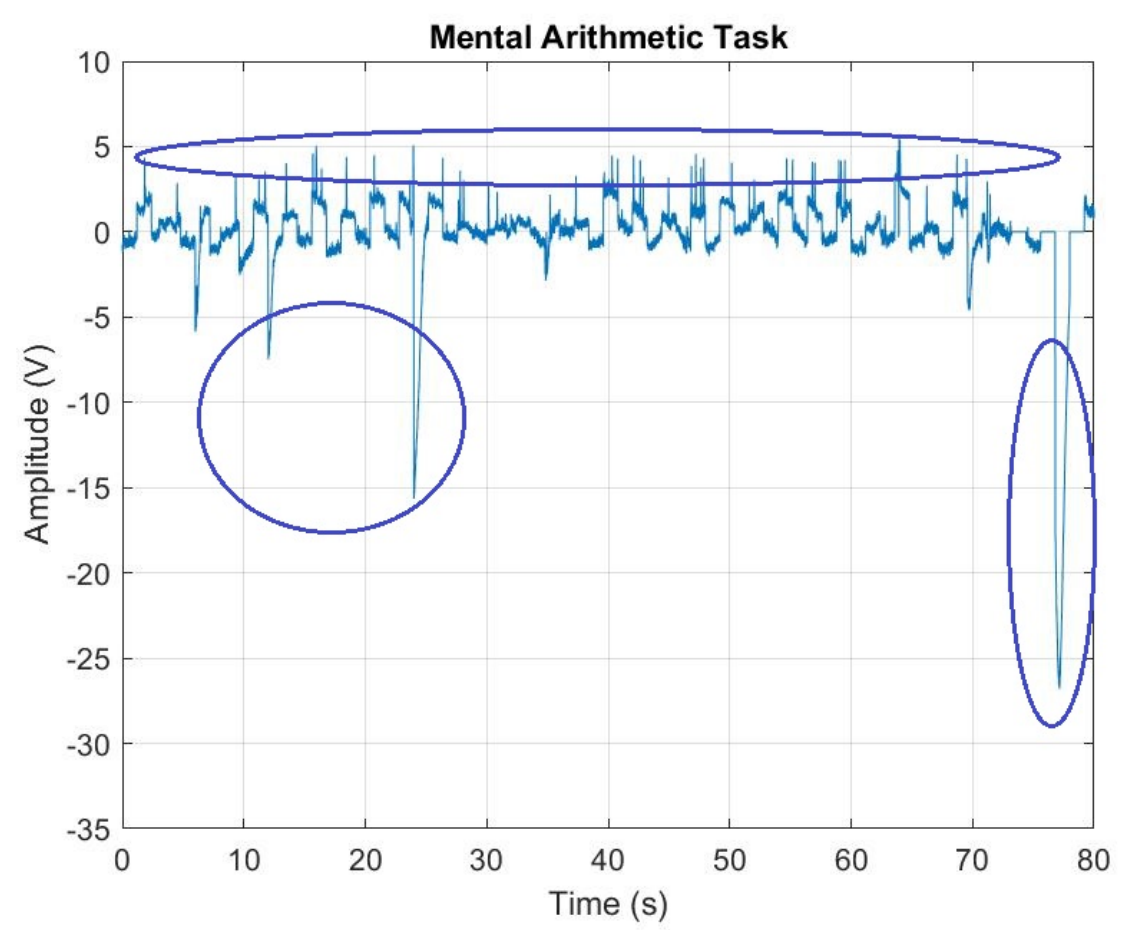

Figure 3.22: Amplitude of mental task data with peaks and troughs highlighted

Fig. 3.23 and fig. 3.24 show the difference between high cognitive load and low cognitive load when the signals are filtered into each brainwave bandwidth. These plots are the same scale and show that rest data produces fewer peaks in all regions when compared to mental arithmetic task data, other than delta which is undetectable in both. This is another demonstration of the amplitude of the data relating to a unique audified signal. When a subject is undertaking a cognitively demanding task, EEG will detect an in increase in theta, alpha and beta brainwaves. This is in line with relevant literature $[104,105,106,101,107]$ and shows that this method of audification is sensitive to changes in cognitive load. 


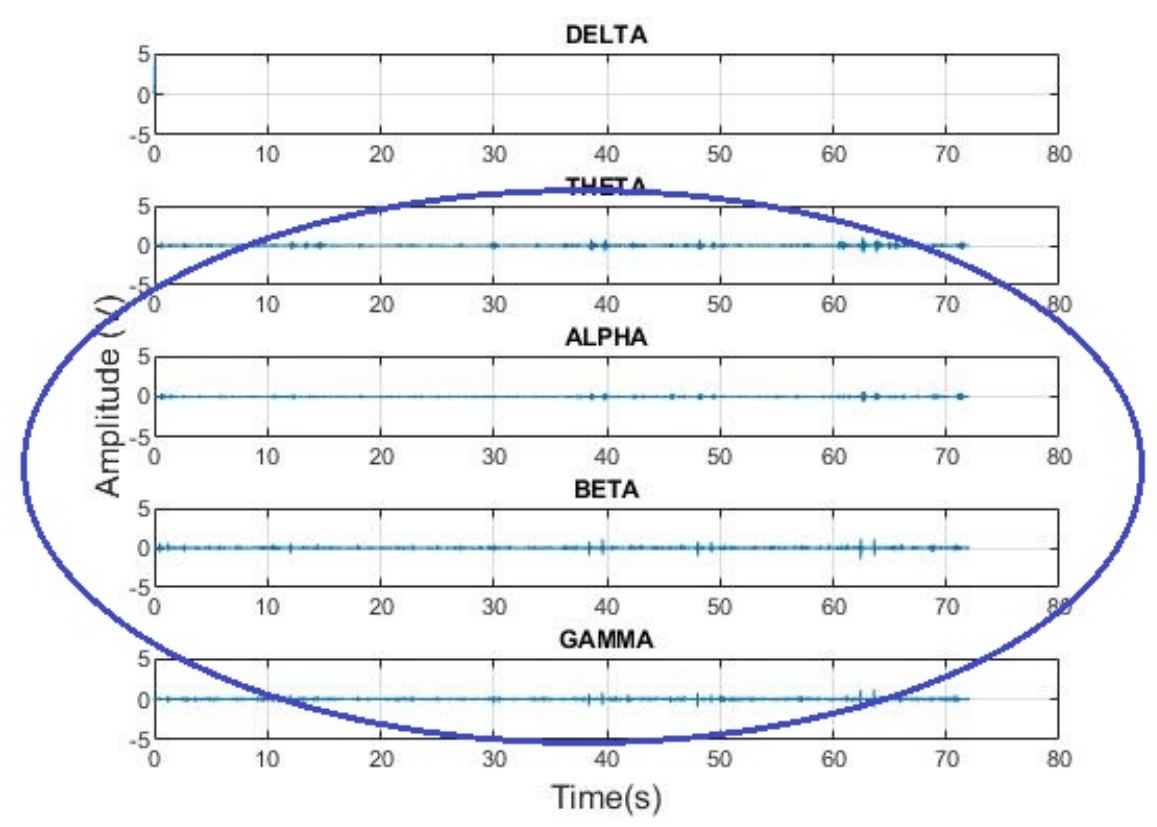

Figure 3.23: Filtered mental rest data showing comparatively less prominent signals in each brainwave bandwidth 


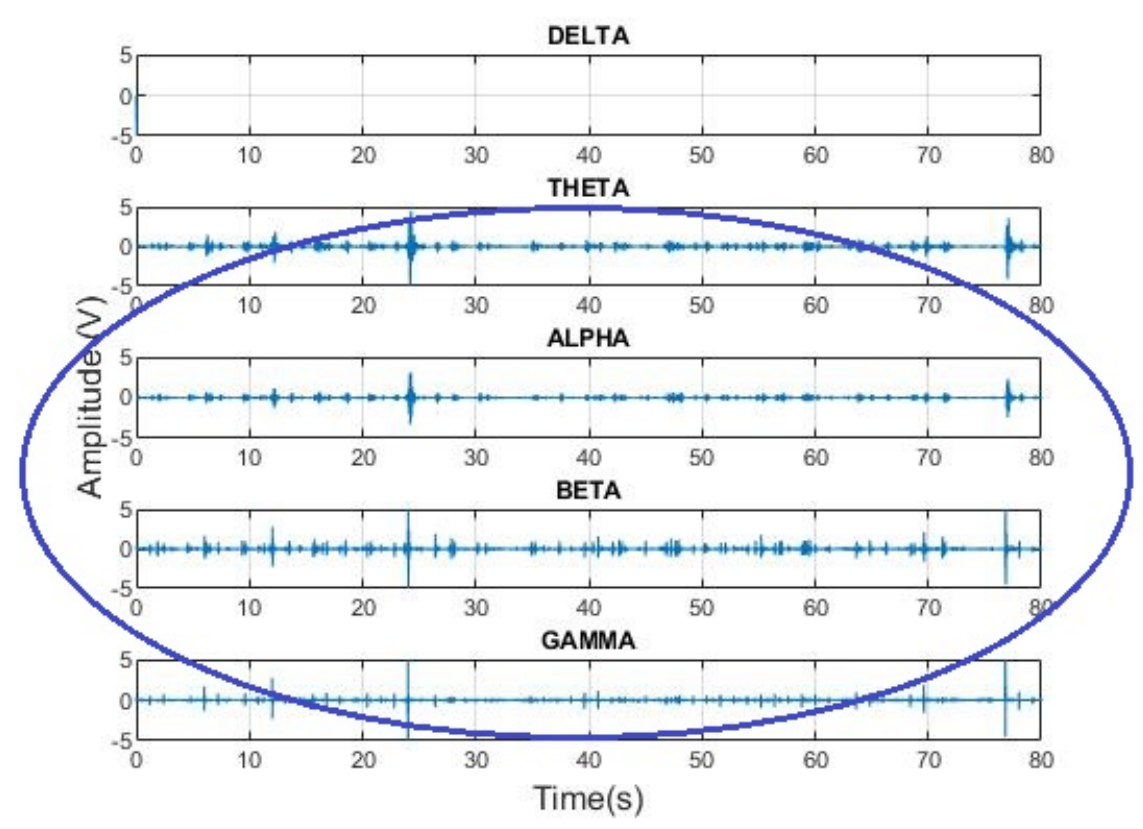

Figure 3.24: Filtered mental arithmetic data showing comparatively stronger and more unique signals in each brainwave bandwidth.

\subsubsection{System Error Detection}

While analyzing the dataset, it was evident that the data from some subjects exhibited erroneous variation. One possibility is the data collection process for these participants was faulty, caused by a loose connection or temporary fault in the software. Originally it was planned to not use these, but the initial analysis showed that these data, although flawed, allowed for valuable analysis. Below (fig. 3.25, fig. 3.26, fig. 3.27, and fig. 3.28) you can see the difference in magnitude of the erroneous data compared to the expected data. It appears that the data may start as flawed (fig. 3.25 and fig. 3.26) as seen by the section of larger amplitude at twenty-five seconds and below which then returns to the nominal range. Fig. 3.27 seems to exhibit expected 
behaviour when compared to fig. 3.20, suggesting that the section of collection pertaining to this data may have been performing as expected. Fig. 3.28 seems to have a lot of its data cut off at positive integers, whilst still exhibiting negative amplitudes and having an overall smaller amplitude and variance in data when compared to fig. 3.22. Although these data show different kinds of unexpected behaviour that could be caused by any error during the collection phase, they do show that outlying data can be observed. A combination of time series plots and audification allow for detection of system errors early on in the analysis or evaluation of the participant.

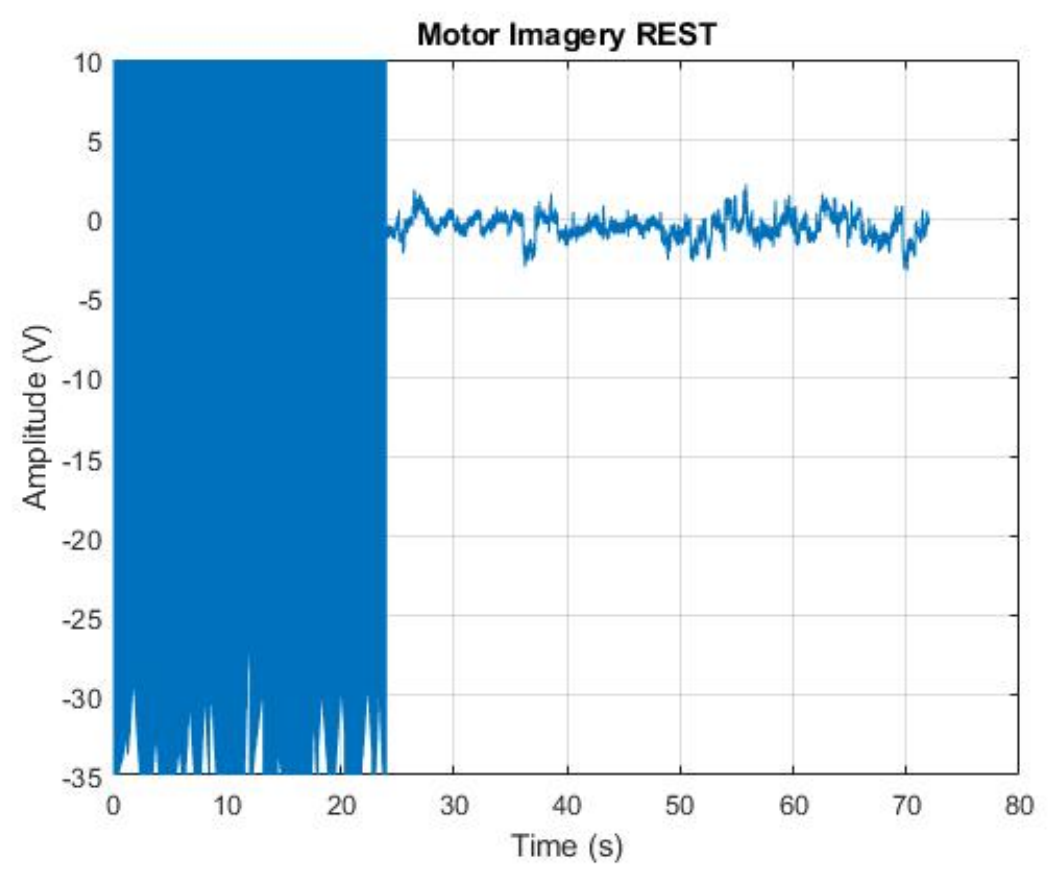

Figure 3.25: Erroneous data for motor rest data. 


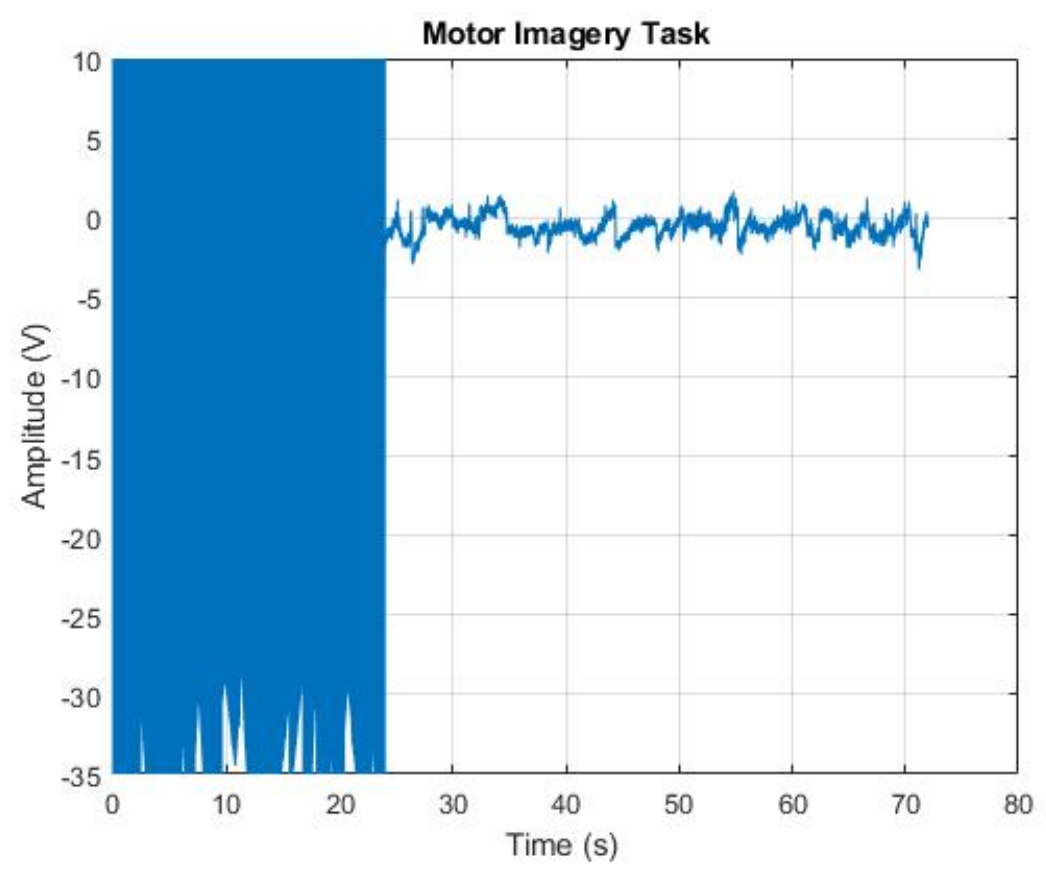

Figure 3.26: Erroneous data for motor imagery task data.

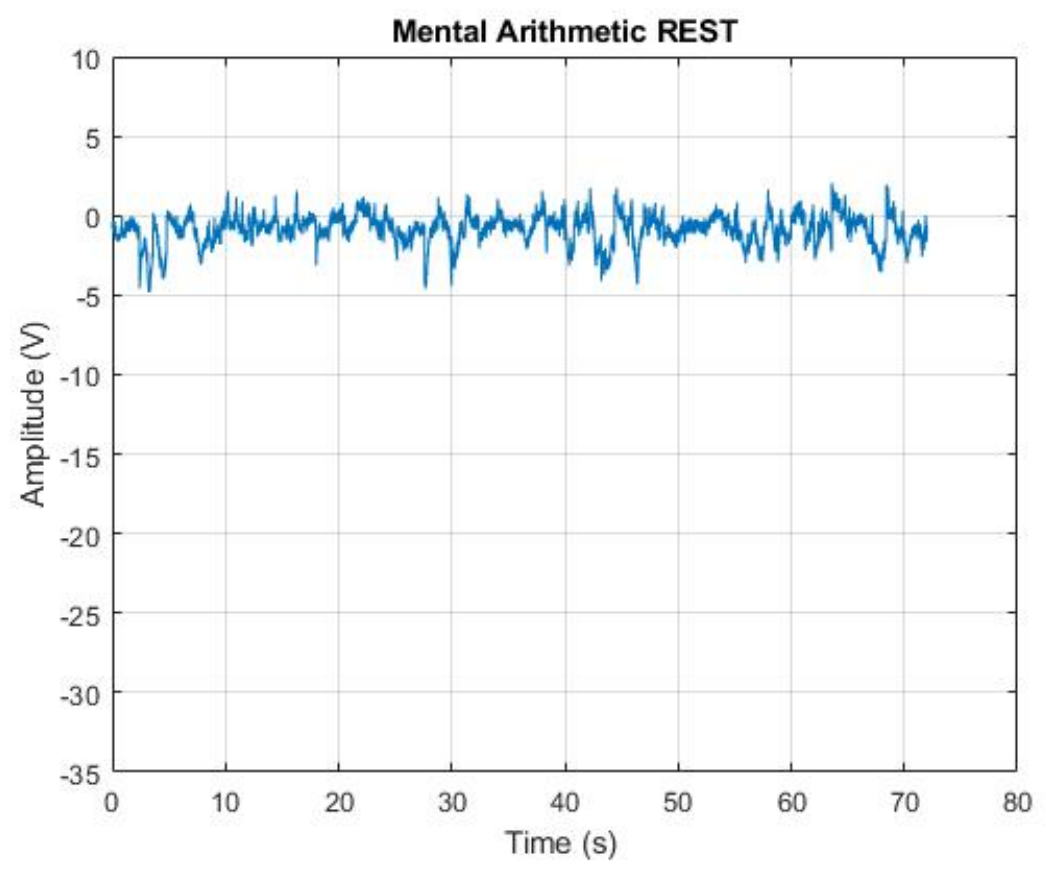

Figure 3.27: Erroneous data for mental rest data. 


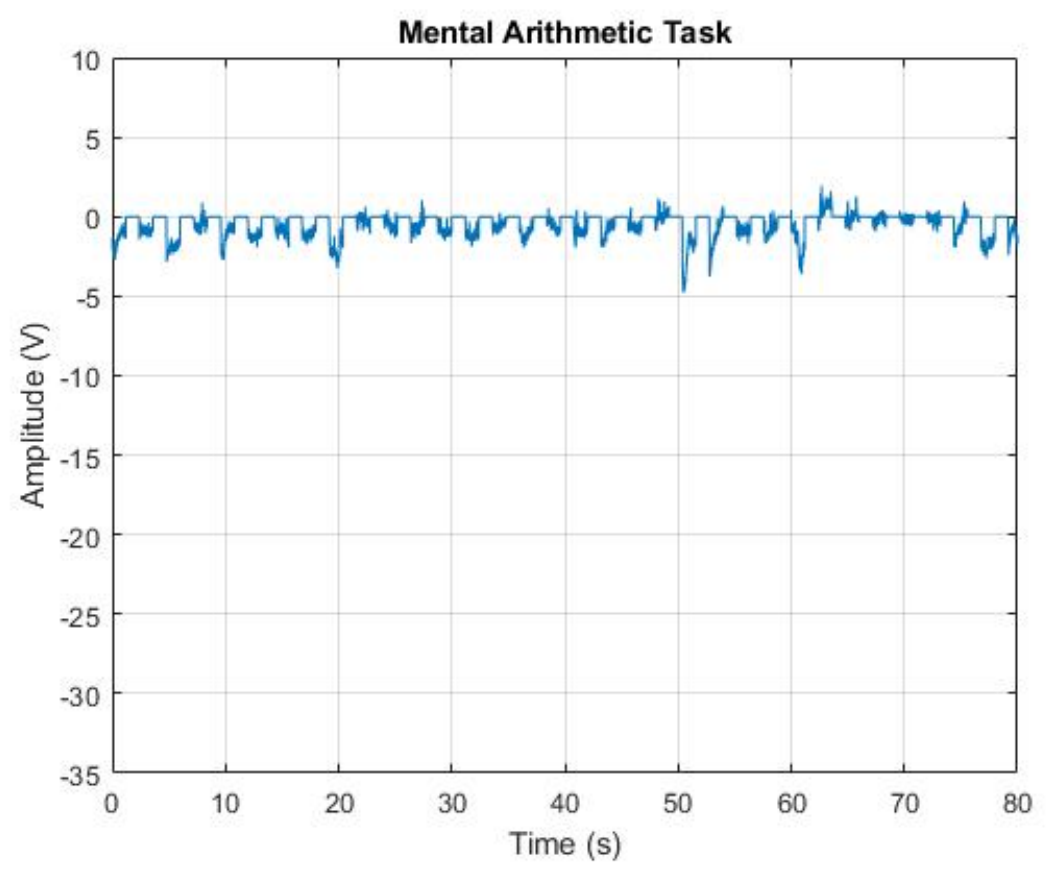

Figure 3.28: Erroneous data for mental arithmetic task data.

\subsubsection{Summary}

In summary, twenty-nine sets of WAV files were generated by filtering data from open-source data set into the gamma bandwidth and then compression by a factor of five, resulting in a file with only the gamma region EEG data at a sampling frequency of $5000 \mathrm{~Hz}$. Each set of audio files pertaining to one of the twenty-nine original participants consisted of four audio files; one for motor imagery task data, one for mental arithmetic task data and one for each rest section, related to the tasks. This resulted in 116 files that were then trimmed down to ten seconds to facilitate audification of the core data in each task section.

In the next section these files were used to evaluate whether novice participants were able to detect differences in cognitive load by listening to audified EEG data. 
This was tested by constructing a survey where participants were asked to listen to audio samples generated by audifying EEG data relating to different tasks and different levels of cognitive load.

\subsection{Survey}

\subsubsection{Purpose}

The survey was developed to evaluate the use of audified EEG to detect differences in cognitive load by novice observers.

\subsubsection{Survey Methodology}

To be recruited to participate in the survey, people had to be over the age of 18 with no hearing impairment. Participants included students at Queen's University and the public. An advertisement was sent out by a survey link sent out from the Building and Designing Assistive Technology (BDAT) lab social media (Twitter, Facebook) as seen in Appendix D.1. The link contained a Letter of Intent (LOI - Appendix E) and a link to the survey, hosted by Qualtrics (Provo, Utah, United States). The entire survey was done using Qualtrics, which anonymizes and compiles the results. This study was approved by Queen's University Graduate Research Ethics Board (GREB) (Appendix F).

Once informed consent was given, participants were asked to listen to the audio files generated from the EEG dataset [3]. The survey was estimated to take no longer than 20 minutes. Section 1 entailed listening to four audio samples of ten seconds each, and section 2 entailed listening to a total of sixty audio samples of ten seconds each. This equates to just over ten minutes of listening time, so twenty minutes was 
provided as the expected time to complete the study.

The data used for section 1 of the study was collected from participant 1 of the dataset. This is the same data used for the plots and graphs throughout this thesis. For section 2 of the study, twenty questions were provided to the listeners. While data from 29 participants were available, there was a possibility of participant fatigue when listening to too many samples. It was determined that twenty would be sufficient to enable effective statistical confidence. This also allowed for some minimal randomizing. A random number generator was used to generate twenty numbers between 1 and 29. These numbers were used to choose which participants' data would be used for each question.

The study consisted of two sections. The first section asked participants to listen to four audio files and identify the odd one out. There was no explanation of the theory before this task. The first section was intended to prepare participants for the types of audio files they were expected to listen to, while ensuring that individuals were able to select a unique audified sample with minimal instruction. The odd one out represented the higher cognitive load. There was only one question in this section. The question from section 1, as it appeared on Qualtrics, can be seen in fig. 3.29

The second section was designed to evaluate whether participants could correctly assess cognitive load based on sound alone. There was minimal instruction given, hoping to confirm that even untrained people have intuition as to what the brain sounds like during higher cognitive load. There was a brief explanation of cognitive load before the questions: "The sound that was different in the previous question was the one file associated with high cognitive load. Cognitive load essentially refers to how hard your brain is working. High cognitive load means that the brain is working harder 


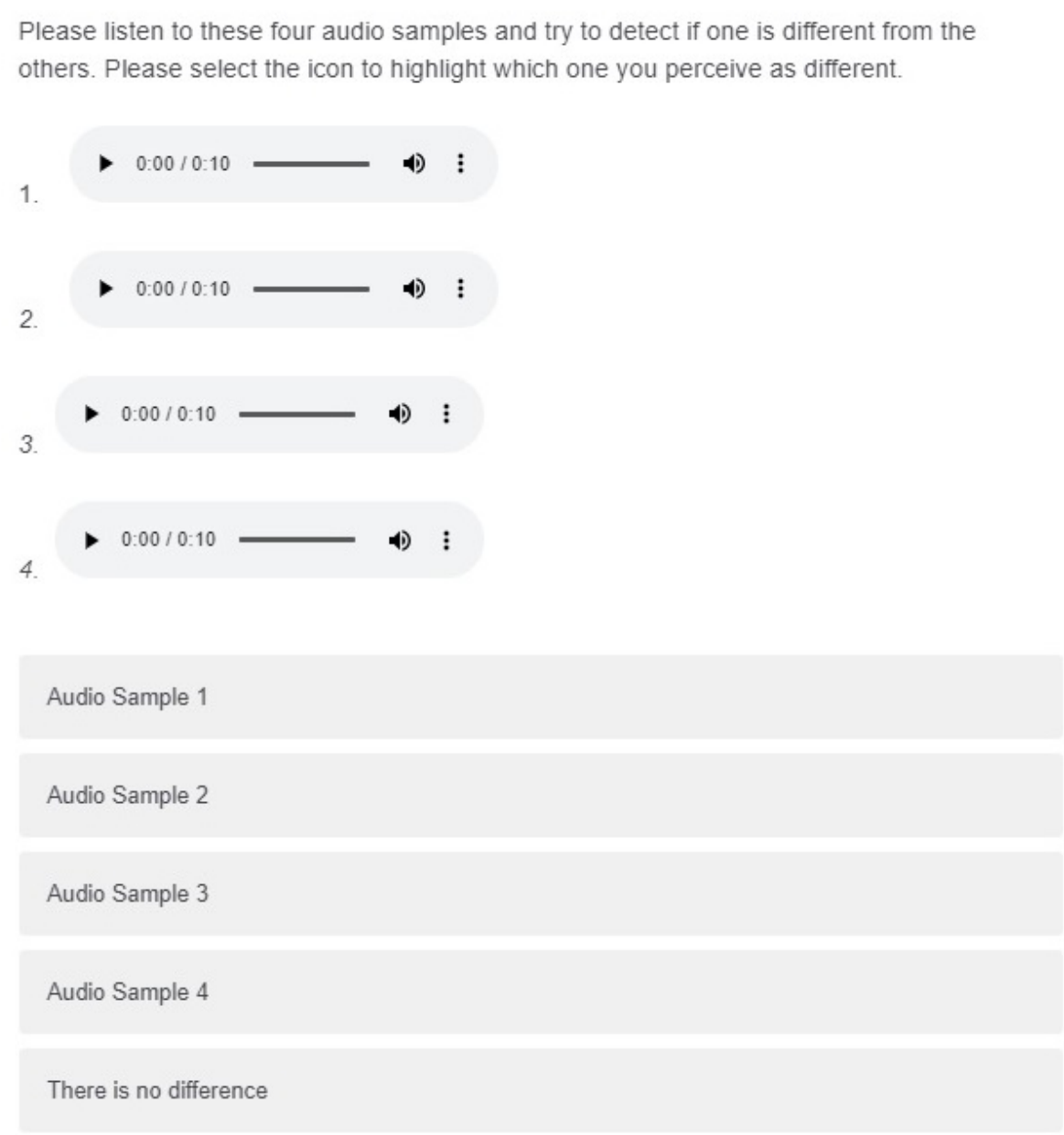

\section{Figure 3.29: Section 1 of the Qualtrics Survey.}

processing information, such as when focusing hard on an arithmetic task. Now I am going to ask you to go through a series of grouped sounds. One audio sample will be associated with higher cognitive load than the other." The second section of the study included 20 questions. Each question required participants to listen to three audio files and identify the option that appeared to be associated with higher cognitive load. Three audio files were chosen, one related to each type of task; motor imagery, mental arithmetic, and rest. Two audio files of rest data (as there was in section 1) 
was deemed unnecessary as it may lead to fatigue when repeated twenty times. In total, there were 20 questions (60 audio files) comprised of mental arithmetic, rest, and motor imagery data. The participants were able to listen to each audio sample as many times as wanted before answering. Fig. 3.30 shows the construction of the survey. An example of the questions from section 2, as it appeared on Qualtrics, can be seen in fig. 3.31

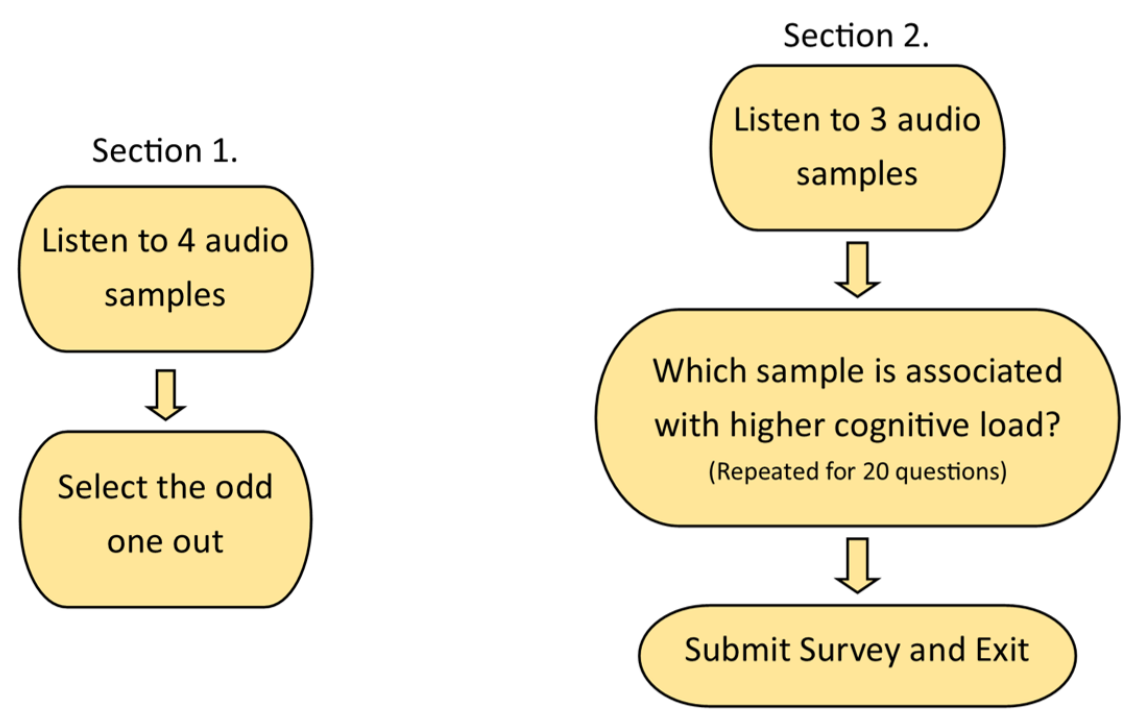

Figure 3.30: Structure of the survey consisting of section 1 and section 2 . 


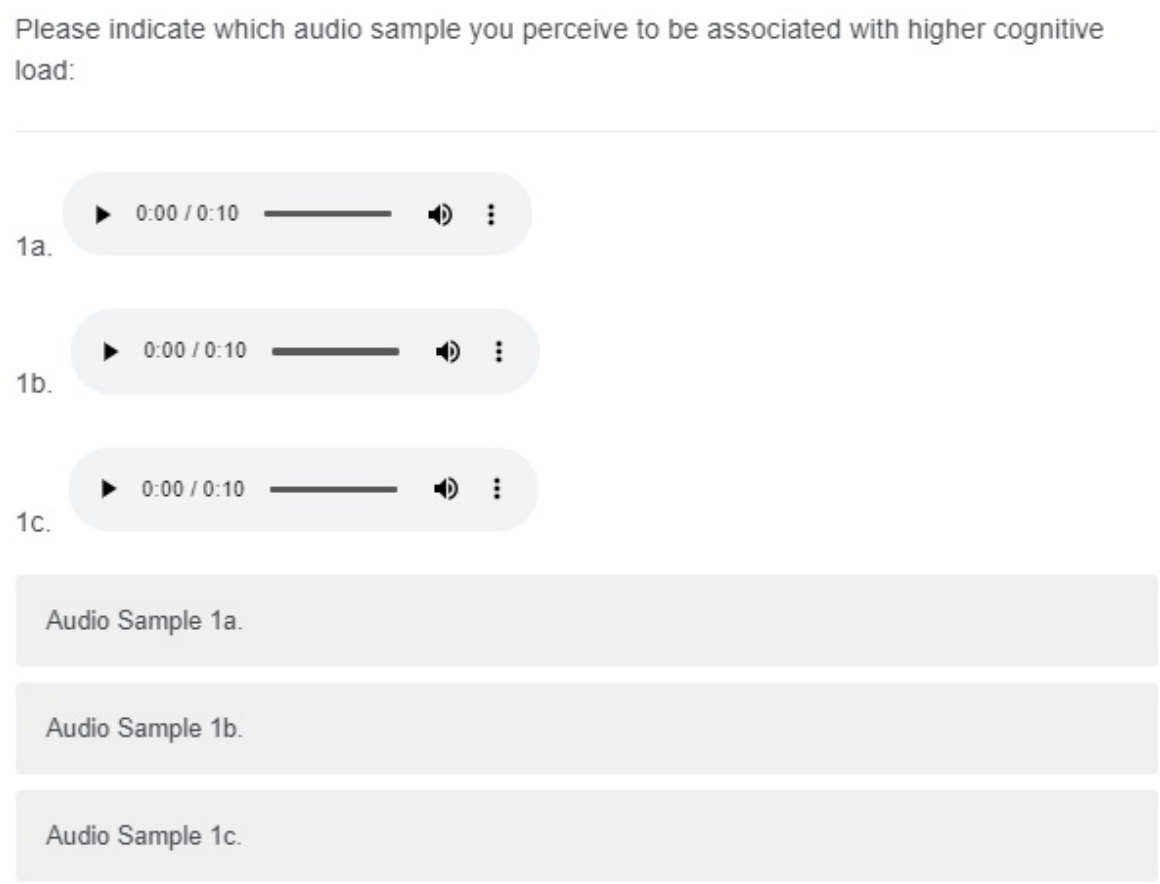

Figure 3.31: Question 1, of section 2, of the Qualtrics Survey.

\section{Anticipated Risks and Discomfort}

The survey entailed little to no risk, although there may have been some unpleasantness due to listening to a large amount of samples. The participant was able to listen using earphones or speakers, and control volume as desired by controlling output of their computer. This study was conducted remotely, so there was no danger from interacting with other participants. One potential hazard was the volume being too high and damaging a participant's hearing. To reduce this risk to a minimum, there was a warning at the beginning of section two: "PLEASE TURN VOLUME DOWN ON YOUR DEVICE. IF YOU CANNOT HEAR ANY SOUND AT ALL, PLEASE EXIT AND TRY USING A DIFFERENT DEVICE. Then adjust volume on your device to the appropriate level for this survey." 


\section{Chapter 4}

\section{Results}

\subsection{Survey Results}

Seventy-five participants consented to taking part in the study; only 56 participants answered section 1. This is $74 \%$ of the original number. A total of thirty-eight participants finished section 2, completing the study in its entirety. This is just over $50 \%$ of the original 75 participants. Only $67 \%$ of participants who completed section 1 continued to complete section 2 and finish the study. This is possibly due to confusion about the concept, or fatigue caused by repetitive questions. There were also some issues with audio not playing on certain devices.

Section 1 of the study asked participants to listen to four audio samples and select the odd one out, or state that there was no difference. The odd one out was an audio sample generated by the mental arithmetic data, whereas the other files were associated with rest data and motor imagery data. In this instance the 'correct' answer, and answer we were expecting participants to select was sample 3. Results from section 1 are summarized in table 4.1 . 


\begin{tabular}{|c|c|c|c|c|c|c|c|}
\hline Sample & Sample & Sample & Sample & No & Total & Percentage & Chance of guessing \\
\hline 1 & 2 & 3 & 4 & difference & & correct & correct \\
\hline 1 & 0 & 47 & 2 & 5 & 55 & $85.45 \%$ & $20 \%$ \\
\hline
\end{tabular}

Table 4.1: Results from Section 1 of the survey.

Section 2 asked participants to select the audio file they perceived to be associated with higher cognitive load. There was a choice of 3 audio files, where one was associated with mental arithmetic, one was associated with rest data, and one was associated with motor imagery data. There were 20 questions of this type, with each question containing data from a different subject of the online dataset, as shown in fig. 4.1 and table 4.2. Numerical details of the results for section 2 can be seen in Appendix G.1.

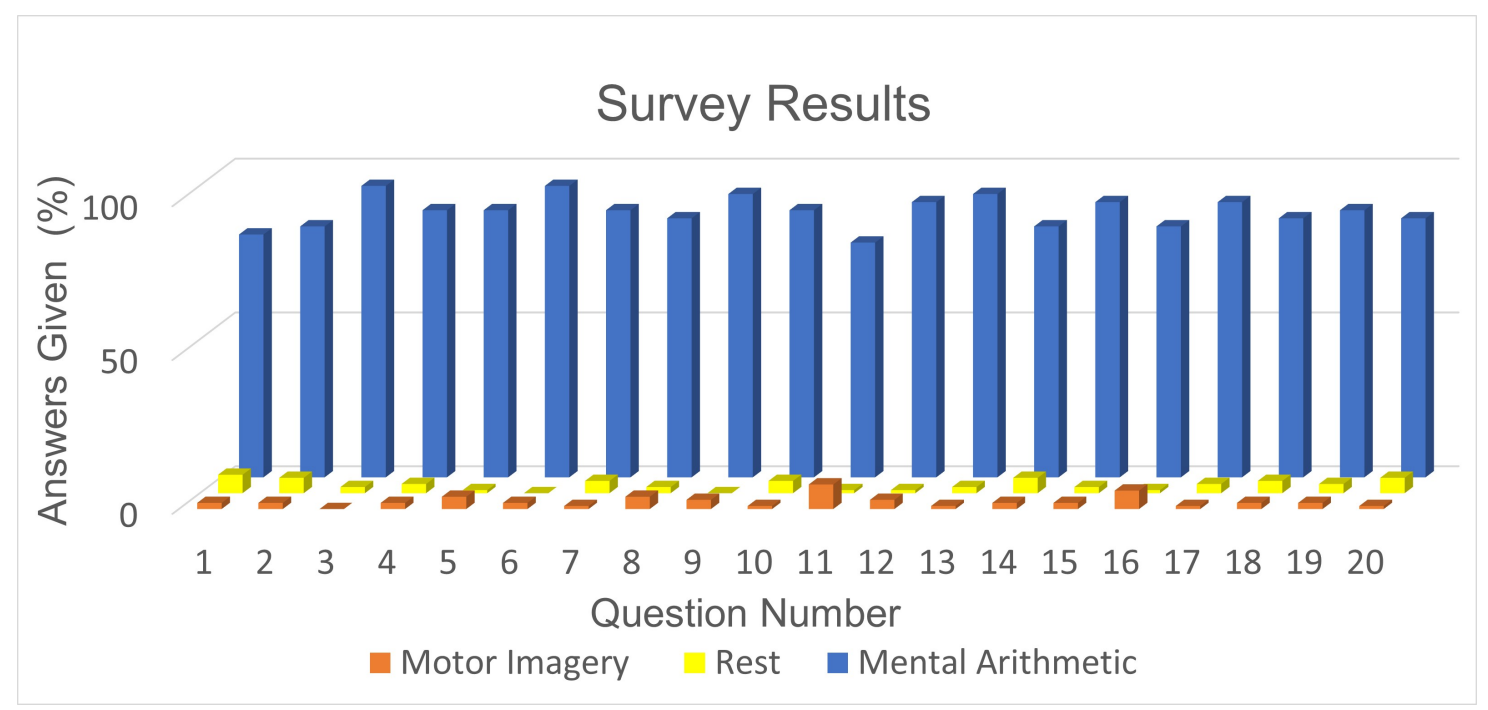

Figure 4.1: Showing the percentage of answers selected for each question. Mental Arithmetic (blue) is the correct answer. 


\begin{tabular}{||c|c|c|c||}
\hline Correct answer & $\%$ & Question number & Dataset participant \\
\hline \hline Average & $\mathbf{8 6 . 4 5}$ & & \\
\hline Lowest correct & 76.32 & 11 & 17 \\
\hline Highest correct & 94.74 & 3,6 & 6,9 \\
\hline Difference & 18.42 & & \\
\hline Chance of guess & 33.33 & & \\
\hline
\end{tabular}

Table 4.2: Average responses from Section 2 of the survey.

\subsubsection{Inter-Rater Reliability}

Inter-rater reliability assesses the level of agreement between the participants of the study. For this study the Fleiss' Kappa was used [127], measuring the agreement between more than two raters, where agreement due to chance is factored out. Fleiss' Kappa was used to determine how consistently different participants correctly identified high cognitive load using the audified EEG signals. Values closer to one indicate better agreement between raters. Table 4.3 shows the suggested interpretation of kappa values [128]. 


\begin{tabular}{||c|c||}
\hline Kappa & Interpretation \\
\hline \hline$<0$ & Poor agreement \\
\hline $0.01-0.2$ & Slight agreement \\
\hline $0.21-0.40$ & Fair agreement \\
\hline $\mathbf{0 . 4 1}-\mathbf{0 . 6 0}$ & Moderate agreement \\
\hline $0.61-0.80$ & Substantial agreement \\
\hline $0.81-1.00$ & Almost perfect agreement \\
\hline
\end{tabular}

Table 4.3: Inter-Rater reliability depending on values of Kappa.

Fleiss' Kappa is calculated using the eq. 6.1 below:

$$
k=\frac{P o-P e}{1-P e}
$$

Where $\mathrm{k}=$ the Fleiss' Kappa result,

Po $=$ observed agreement $=$ proportion of agreeing units.

$\mathrm{Pe}=$ expected agreement $=$ proportion of units for expected chance agreement

The observed value, Po, is calculated by summing the square of all the observed values. Pe is the same calculation compared to the expected value, and in this instance it would be 38 because we would expect that everyone agrees on the same selection.

From this, we calculated a Kappa of 0.56 which shows a moderate agreement. 


\subsection{Alternative Data}

We aimed to confirm that this method functions with other datasets. However, we found it very difficult to obtain open-source datasets that consisted of appropriate data. It appears that open-source datasets of EEG collection focus on motor movement and motor imagery but lack any mental arithmetic data. Another issue with open-source datasets is the difficulty of extracting the data into useful segments. All the datasets that were obtained contained continuous streams of data that were comprised of multiple tasks, rest sections, and baselines. They did describe how the data were laid out, but it was not clear enough to confidently separate the data into task and rest, for use with this method. The dataset used for the survey was the only data available to the researcher that allowed data segmentation for the method proposed herein. 


\section{Chapter 5}

\section{Discussion}

\subsection{Audification Process}

One key observation from preliminary assessment of audifying EEG was that mental arithmetic data exhibits a significantly higher cognitive load than motor imagery data. This is supported by other research into cognitive theory, stating that the brain needs to process multiple parts of information to illicit a high cognitive demand. In this research, the importance of significantly high cognitive load is displayed in both the visual and audible medium with mental arithmetic data generating a distinct audified signal, that can be detected by untrained individuals.

\subsection{Survey}

The results from the study show that untrained participants were able to detect a difference in cognitive load by listening to an audified sample of EEG data. On average there was an $\mathbf{8 6 \%}$ success rate of selecting the correct audio sample. Each of the 20 questions for section 2 used data from a different subject from the online dataset. This was randomized but each participant of the study listened to the same subject on the 
same question. This allowed us to see variance among subjects i.e., were some people's audified signals easier to identify than others? The highest percentage of correct answers was $\mathbf{9 4 . 7 4 \%}$, with the lowest at $\mathbf{7 6 . 3 2 \%}$. Current methods of concussion assessment fail to report up to $50 \%$ of concussions [6] due to misunderstanding of symptoms and individuals intentionally avoiding diagnosis $[7,8,129]$. The results of this research serve as a proof of concept to assess concussion. We have demonstrated that untrained individuals can detect changed in cognitive load through audified EEG. We believe this method can be utilized to detect the abnormal neurological behaviour consistent with concussion.

Our results show a success rate of at least $76 \%$, which is a significant improvement on the $50 \%$ success rate of current concussion diagnostic tools. EEG itself has been shown to be greater than $90 \%$ accurate when assessing concussive head injuries [82]. By combining EEG with detection of audified data, it is hoped that there can be improvement on accurate diagnoses as well as ease of use. The objective tool outlined in this research would allow for assessment without extensive knowledge of brain signals or EEG and would reduce the likelihood of someone intentionally skewing the results. If this method could be used on individuals suspected of a head injury, the chance of unreported [6], undiagnosed, and repeated injuries would decrease.

The results of table 6.1 and table 6.3 suggest that the survey participants did not simply guess an answer and were confident in their responses. In section 2 there were three possible answers. That means we would expect a correct guess percentage of 33.33 from guessing alone. The lowest percentage of correct answers is $\mathbf{7 6 . 3 2 \%}$. This allows us to ascertain that there is significance in the results of this study and it is not due to luck, but is due to correct assessment. 
An inter-rater reliability found a Fleiss' Kappa of 0.56, translating to a moderate agreement. Assessment of agreement would be more insightful with a larger pool of participants, and it is hoped that the level would improve.

During the study, we received feedback from some participants that can be summarized into negative (-) or positive (+):

- The explanation was confusing.

- The samples were a bit long and unpleasant.

- The concept was confusing.

+ The samples could have been shorter and still sufficient to ascertain a difference.

+ The difference between the samples is discernible right from the start.

There are certain elements that indicate further investigation and refinement would enable and increased success rate. The issues that would have to be address are here:

1. The sounds observed in this research are unpleasant [130] and unfamiliar to most people, possibly leading to participants being disoriented, or in a rush to finish. The samples used were ten seconds long. Perhaps ten seconds of audified EEG is too long to keep participants engaged.

2. Between sections one and two the participants were given an explanation of cognitive load which may have resulted in some confusion about the identification of the distinguishable sound. 
Seventy-five participants consented to take part in the study. Only 56 participants completed Section 1. Only 38 participants completed Section 2, finishing the study. At the beginning of section 2, a brief description of cognitive load was provided relative to section 1. Anecdotal evidence from people who completed the study and further reached out to the researcher suggests that they found this description confusing. This supports the suggestion that participants were confused by the original explanation of the study and either chose not to continue or did not understand the instructions.

This study focused on the audification of different levels of cognitive load, aiming to show that this method can be applied to detecting concussion. It has been demonstrated that this method is sensitive to the changes in brain activity associated with increased cognitive load (fig. 3.23 and fig, 3.24). There are many studies reporting how concussions can influence EEG measurements, such as increased theta amplitude and decreased gamma frequency [86, 10, 81, 82, 83, 84, 85, 131, 132, 133, 134, 135]. In an earlier study by Larsson (1956) it was shown that EEG displays reduced overall amplitude and irregular theta activity in boxers 15-30 minutes after a fight, with the results being even more prominent when the fighter was knocked unconscious [136].

\subsection{Practical Application}

The audification algorithm used in this research is easily to replicate as it can be used with common file formats used for recording EEG data. The specific timings and types of data can be changed to fit another dataset, as long as there is sufficient detail to match specific data with its time stamp within the dataset. There could be improvements, especially if data was recorded specifically for this approach to make the process more automated. However; the process of audification was not 
the focal point of this research. The purpose is to demonstrate a proof of concept where audifying EEG data can be applied to concussion. The specific process of audification is bound to change significantly in further research, but we did manage to demonstrate that there is promise in using audification to assess cognitive load. This is an application of audification that is not being widely utilized.

This study outlines a method of audification that is successful at detecting a difference between high and low cognitive load. The process described here uses EEG to detect the activity of the brain in terms of frequency and amplitude. The changes of amplitude and frequency are the driving factors of the audification process. This shows that the method of audification described here can be applied to any state of the brain where the amplitude and frequency of EEG are influenced. Cognitive load and concussions both influence brain activity as seen through EEG, suggesting that this method of audification can be applied to detecting a concussion. Overall amplitude and gamma activity decrease in a concussed brain, so it is suggested that by comparing high cognitive activity in a healthy brain to high cognitive activity in a concussed brain, there will be a discernible difference when the signals are audified.

It is suggested that this method could be used to establish a baseline of neurological activity of individuals. This could be done my a team medic on a sports team. If a member of that team is injured, this method could be used to compare their current neurological activity with their baseline and healthy activity. If an injury is discovered, treatment and recovery can be recommended. The same method could be used for post-injury checks to assess the recovery of this individual. This would ensure that the individual does not return to play before they are fully recovered. Audified outputs of each stage could be recorded and shared with different medical 
practitioners. The objectivity of this method would allow for continued checks of an individual's brain health, even by multiple parties.

It is important to be careful when using this method with injured participants. Applying this method may rely on participants taking part in tasks of a high cognitive load. It has been shown that recovery of concussed individuals can be inhibited and impeded if those individuals are exposed to cognitively demanding tasks before appropriate recovery [50]. However this method may be applied, the health and safety of the participants is a priority. A safer method would be to improve this method to detect a difference between the resting state EEG patterns of healthy individuals compared to injured individuals. This would allow for the comparison of audified EEG data, related to concussion, without putting anyone at further risk or lengthening recovery periods.

Throughout this research we have discussed the shortcomings of current concussion detection and treatment, whilst highlighting how urgent it is to address. Concussion seems to be consistently underestimated and underplayed. Whether or not this research is used directly to aid in the detection of concussions, the overarching goal is to raise awareness of the danger of concussion and offer different approaches to give insight into the health of one's brain. There are a variety of tools available and the majority of brain function is still an elusive mystery. We have shown there are ways to gain insight to the activity of the brain and thus evaluate issues at the source. The importance of the brain's regular function and the capacity for the body in general to warn us of an issue is not one to take lightly; we just have to know where to look and how to be receptive to these warnings. 


\subsection{Issues and Shortcomings}

This research has only been successfully tested on one dataset suggesting that the method proposed here is dependent on the way this specific data was collected. The method proposed here requires EEG data to be separated in a way that is not common practise when collecting EEG data, but data can easily be transferred into appropriate formats. The original plan was to collect our own EEG data on participants, but this was not possible due to COVID-19 restrictions. The method proposed in this research facilitates recording and playback of brain activity, it does not allow for real-time assessment. It does allow for assessment for data that could be carried out immediately after the collection process. This would allow for an overall assessment, in a similar time frame to current concussion detection methods.

The biggest issue with this research is experimenter bias. Initial assessment and selection of audio files was carried out by the researcher themselves, and was not appropriately blinded. Due to the pandemic, it was difficult to seek out the involvement of colleagues to further insight. This work provides the methods and procedures based on judgement calls at the time of research. The details in this research will allow anyone pursuing this work to adapt it to ways they see fit, and experiment with tighter controls to move the application of audification in a direction that is beneficial to many. 


\section{Chapter 6}

\section{Future Work and Conclusions}

\subsection{Future Work}

The most important first step in future work is to collect more data. This collection of data should compare cognitively demanding tasks to tasks that are less demanding. It would be ideal to immediately record the data in a separated way by, for example, recording three minutes of a demanding task, and then three minutes of rest, the data could then be reliably separated. This would allow for audification of the tasks separately, to then be trimmed to account for the beginning and end of the tasks. It would also be ideal to combine multiple increments of similar tasks in one dataset. This would allow for a longer collection time of data, which can be compressed to a greater amount.

The study revealed that untrained participants may be confused by explanations of technical concepts, such a cognitive load, which may lead to incorrect detections

and non-compliance. To ensure ease of use, it is important to keep explanations as simple as possible to avoid confusion or provide appropriate levels of understanding for the concepts involved. It is also recommended to reduce the length of the audio 
samples. Ten seconds per audio file seemed more than enough time to detect changes in the audible signal. It would also be ideal to vary the questioning to minimize fatigue or disinterest in listening to sixty similar audio files in a row. All these considerations can maximize adoption of audification.

This method can be improved to enable differentiation using audible signal between a concussed and a healthy brain. Audible difference is already present in different tasks such as mental arithmetic and rest. A concussion may create additional variances in the data. For example, a concussion may lead to an audible difference between low and high cognitive load that is less prominent in a concussed brain due to the decrease in amplitude and gamma activity. It is suggested that EEG is used to measure activity of the brain during tasks of high cognitive load on an individual before, during, and after they are concussed. We predict that the influence of the concussion on the EEG output will be discernible through audification.

\subsection{Conclusions}

To detect cognitive load through audification, the task needs to be of sufficiently high cognitive load and compared to a task of lower cognitive load, such as rest or a task that is less demanding, such as motor imagery. The high cognitive load task needs to be one that encourages the individual to use many resources and process different concepts. Mental arithmetic tasks that involve a participant processing and memorizing data, such as complex mathematical equations are ideal.

This audification method is valuable even if the data is flawed. A familiarity with what to expect allows detection of an issue. The audified sound would likely be louder or more erratic than expected, warning of a poor connection or error in the 
translation, which is advantageous over plots.

Audification has not been well studied. This research shows how the application of audification of SMR can be used to assess activity of the brain in near real-time.

There is a need for new and accessible methods of determining the existence of a concussion. This research has shown that it is possible to detect different levels in cognitive load through audification alone. This was carried out by untrained individuals with minimal explanation of the concepts involved in the task. This is an important step in providing an alternative method for assessing brain activity. With more research this method can be applied to assessing the risk of, or diagnosing, a concussion.

The danger of concussions is becoming more apparent, so it is important to provide solutions that can compete with the current methods. The method proposed here relies on the raw electrical signals detected directly from the brain, conveyed in a more objective way, that can be detected by anyone with functioning hearing. By providing alternative and more effective methods of detecting concussion, researchers and practitioners can reduce the likelihood of undetected concussions. This will directly reduce the chance of repeated concussions, that lead to severe and long-term damage. 


\section{References}

[1] Muse, "A deep dive into brainwaves: Brainwave frequencies $\quad$ explainedl." https://choosemuse.com/blog/ a-deep-dive-into-brainwaves-brainwave-frequencies-explained-2/, Jun 2018. Accessed on 2021-02-14.

[2] J. Malmivuo and R. Plonsey, Bioelectromagnetism. 13. Electroencephalography, pp. 247-264. 1995.

[3] J. Shin, A. v. Lühmann, B. Blankertz, D. Kim, J. Jeong, H. Hwang, and K. Müller, "Open access dataset for eeg+nirs single-trial classification," IEEE Transactions on Neural Systems and Rehabilitation Engineering, vol. 25, no. 10, pp. 1735-1745, 2017.

[4] N. B. I. Association, "Brain injury statistics." https://www.nbia.ca/ brain-injury-statistics/, Dec 2019. Accessed on 2021-02-14.

[5] A. B. Peterson, L. Xu, J. Daugherty, and M. J. Breiding, "Surveillance report of traumatic brain injury-related emergency department visits, hospitalizations, and deaths, united states, 2014," 2019. 
[6] K. G. Harmon, J. Drezner, M. Gammons, K. Guskiewicz, M. Halstead, S. Herring, J. Kutcher, A. Pana, M. Putukian, and W. Roberts, "American medical society for sports medicine position statement: concussion in sport," Clinical Journal of Sport Medicine, vol. 23, no. 1, pp. 1-18, 2013.

[7] D. J. Wiebe, R. D. Comstock, and M. L. Nance, "Concussion research: a public health priority," Injury Prevention, vol. 17, no. 1, pp. 69-70, 2011.

[8] M. McCrea, T. Hammeke, G. Olsen, P. Leo, and K. Guskiewicz, "Unreported concussion in high school football players: implications for prevention," Clinical journal of sport medicine, vol. 14, no. 1, pp. 13-17, 2004.

[9] I. J. S. Williamson and D. Goodman, "Converging evidence for the underreporting of concussions in youth ice hockey," British Journal of Sports Medicine, vol. 40, no. 2, p. 128, 2006.

[10] D. A. Hoffman, S. Stockdale, L. L. Hicks, and J. E. Schwaninger, "Diagnosis and treatment of head injury," Journal of Neurotherapy, vol. 1, no. 1, pp. 14-21, 1995.

[11] J. W. Thompson, EEG changes and balance deficits following concussion: one piece of the puzzle, pp. 341-374. Springer, 2006.

[12] S. D. Fickling, A. M. Smith, G. Pawlowski, S. Ghosh Hajra, C. C. Liu, K. Farrell, J. Jorgensen, X. Song, M. J. Stuart, and R. C. D'Arcy, "Brain vital signs detect concussion-related neurophysiological impairments in ice hockey," Brain, vol. 142 , no. 2, pp. 255-262, 2019. 
[13] N. A. Badcock, B. Mousikou, Y. Mahajan, P. De Lissa, J. Thie, and G. McArthur, "Emotiv versus neuroscan: Validating a gaming eeg system for research quality erp measurement," in Front. Hum. Neurosci. Conference Abstract: ACNS-2012 Australasian Cognitive Neuroscience Conference. doi: 10.3389/conf. fnhum, vol. 122.

[14] A. Baeyens and T. Goffin, "Ecj 2012/11, brain products gmbh v. biosemi vof, et al, 22 november 2012 (c-219/11)," European journal of health law, vol. 20, no. 1, p. 93, 2013.

[15] I. Cester, S. Dunne, A. Riera, and G. Ruffini, "Enobio: wearable, wireless, 4-channel electrophysiology recording system optimized for dry electrodes," in Proceedings of the Health Conference, Valencia, Spain, vol. 2123, Citeseer.

[16] M. Daeglau, F. Wallhoff, S. Debener, I. S. Condro, C. Kranczioch, and C. Zich, "Challenge accepted? individual performance gains for motor imagery practice with humanoid robotic eeg neurofeedback," Sensors, vol. 20, no. 6, p. 1620, 2020.

[17] M. Duvinage, T. Castermans, M. Petieau, T. Hoellinger, G. Cheron, and T. Dutoit, "Performance of the emotiv epoc headset for p300-based applications," Biomedical engineering online, vol. 12, no. 1, p. 56, 2013.

[18] C. H. Hillman, M. B. Pontifex, L. B. Raine, D. M. Castelli, E. E. Hall, and A. F. Kramer, "The effect of acute treadmill walking on cognitive control and academic achievement in preadolescent children," Neuroscience, vol. 159, no. 3, pp. 1044-1054, 2009. 
[19] S. A. Imtiaz, Low-complexity algorithms for automatic detection of sleep stages and events for use in wearable EEG systems. Thesis, 2015.

[20] A. Jakab, A. Kulkas, T. Salpavaara, P. Kauppinen, J. Verho, H. Heikkilä, and V. Jäntti, "Novel wireless electroencephalography system with a minimal preparation time for use in emergencies and prehospital care," Biomedical engineering online, vol. 13, no. 1, p. 60, 2014.

[21] J. Katona, I. Farkas, T. Ujbanyi, P. Dukan, and A. Kovari, "Evaluation of the neurosky mindflex eeg headset brain waves data," in 2014 IEEE 12th international symposium on applied machine intelligence and informatics (SAMI), pp. 91-94, IEEE.

[22] S. Kinreich, I. Podlipsky, S. Jamshy, N. Intrator, and T. Hendler, "Neural dynamics necessary and sufficient for transition into pre-sleep induced by eeg neurofeedback," Neuroimage, vol. 97, pp. 19-28, 2014.

[23] O. E. Krigolson, C. C. Williams, A. Norton, C. D. Hassall, and F. L. Colino, "Choosing muse: Validation of a low-cost, portable eeg system for erp research," Frontiers in neuroscience, vol. 11, p. 109, 2017.

[24] V. Mihajlović, B. Grundlehner, R. Vullers, and J. Penders, "Wearable, wireless eeg solutions in daily life applications: what are we missing?," IEEE journal of biomedical and health informatics, vol. 19, no. 1, pp. 6-21, 2014.

[25] K. H. Park, H. J. Kim, B. Oh, M. Seo, E. Lee, and J. Ha, "Evaluation of human electroencephalogram change for sensory effects of fragrance," Skin Research and Technology, vol. 25, no. 4, pp. 526-531, 2019. 
[26] E. Ratti, S. Waninger, C. Berka, G. Ruffini, and A. Verma, "Comparison of medical and consumer wireless eeg systems for use in clinical trials," Frontiers in human neuroscience, vol. 11, p. 398, 2017.

[27] V. Samson, B. P. Kitti, S. P. Kumar, D. S. Babu, and C. Monica, "Electroencephalogram-based openbci devices for disabled people," in Proceedings of 2nd International Conference on Micro-Electronics, Electromagnetics and Telecommunications, pp. 229-238, Springer.

[28] G. Tamburro, D. B. Stone, and S. Comani, "Automatic removal of cardiac interference (arci): a new approach for eeg data," Frontiers in neuroscience, vol. 13, p. 441, 2019.

[29] R. L. Alexander, J. A. Gilbert, E. Landi, M. Simoni, T. H. Zurbuchen, and D. A. Roberts, "Audification as a diagnostic tool for exploratory heliospheric data analysis," International Community for Auditory Display.

[30] W. T. Fitch and G. Kramer, "Sonifying the body electric: Superiority of an auditory over a visual display in a complex, multivariate system," in SANTA FE INSTITUTE STUDIES IN THE SCIENCES OF COMPLEXITYPROCEEDINGS VOLUME-, vol. 18, pp. 307-307, Addison-Wesley Publishing Co.

[31] F. Paas, J. E. Tuovinen, H. Tabbers, and P. W. Van Gerven, "Cognitive load measurement as a means to advance cognitive load theory," Educational psychologist, vol. 38, no. 1, pp. 63-71, 2003. 
[32] E. Johannessen, Measuring Cognitive Load in a Clinical Setting: Medical Learning and Practice. Thesis, 2019.

[33] W. Penfield and H. Jasper, "Epilepsy and the functional anatomy of the human brain," 1954.

[34] Z. Y. Kerr, K. R. Evenson, W. D. Rosamond, J. P. Mihalik, K. M. Guskiewicz, and S. W. Marshall, "Association between concussion and mental health in former collegiate athletes," Injury Epidemiology, vol. 1, no. 1, p. 28, 2014.

[35] B. J. Allen and J. D. Gfeller, "The immediate post-concussion assessment and cognitive testing battery and traditional neuropsychological measures: a construct and concurrent validity study," Brain Injury, vol. 25, no. 2, pp. 179-191, 2011.

[36] D. M. Sosin, J. E. Sniezek, and D. J. Thurman, "Incidence of mild and moderate brain injury in the united states, 1991," Brain injury, vol. 10, no. 1, pp. 47-54, 1996.

[37] G. of Canada, "Concussion: Sports and recreation." https://www.canada. ca/en/public-health/services/diseases/concussion-sign-symptoms/ sport-recreation.html, Oct 2020. Accessed on 2021-02-14.

[38] C. I. for Health Information, "Heads-up on sport-related brain injuries." https: //www.cihi.ca/en/heads-up-on-sport-related-brain-injuries-0, Dec 2018. Accessed on 2021-02-14.

[39] Z. Y. Kerr, J. P. Mihalik, K. M. Guskiewicz, W. D. Rosamond, K. R. Evenson, and S. W. Marshall, "Agreement between athlete-recalled and clinically 
documented concussion histories in former collegiate athletes," The American journal of sports medicine, vol. 43, no. 3, pp. 606-613, 2015.

[40] S. Marshall, M. Bayley, S. McCullagh, D. Velikonja, L. Berrigan, D. Ouchterlony, and K. Weegar, "Updated clinical practice guidelines for concussion/mild traumatic brain injury and persistent symptoms," Brain injury, vol. 29, no. 6, pp. 688-700, 2015.

[41] P. Decq, N. Gault, M. Blandeau, T. Kerdraon, M. Berkal, A. ElHelou, B. Dusfour, and J.-C. Peyrin, "Long-term consequences of recurrent sports concussion," Acta Neurochirurgica, vol. 158, no. 2, pp. 289-300, 2016.

[42] A. M. SMITH, M. J. STUART, D. M. Wiese-Bjornstal, E. K. MILLINER, W. M. O'FALLON, and C. S. CROWSON, "Competitive athletes: preinjury and postinjury mood state and self-esteem," in Mayo Clinic Proceedings, vol. 68, pp. 939-947, Elsevier.

[43] K. M. Guskiewicz, S. W. Marshall, J. Bailes, M. McCrea, R. C. Cantu, C. Randolph, and B. D. Jordan, "Association between recurrent concussion and latelife cognitive impairment in retired professional football players," Neurosurgery, vol. 57, no. 4, pp. 719-726, 2005.

[44] M. Fralick, D. Thiruchelvam, H. C. Tien, and D. A. Redelmeier, "Risk of suicide after a concussion," Canadian Medical Association Journal, vol. 188, no. 7, p. 497, 2016. 
[45] P. McCrory, W. H. Meeuwisse, R. J. Echemendia, G. L. Iverson, J. Dvořák, and J. S. Kutcher, "What is the lowest threshold to make a diagnosis of concussion?," British Journal of Sports Medicine, vol. 47, no. 5, pp. 268-271, 2013.

[46] T. M. Talavage, E. A. Nauman, E. L. Breedlove, U. Yoruk, A. E. Dye, K. E. Morigaki, H. Feuer, and L. J. Leverenz, "Functionally-detected cognitive impairment in high school football players without clinically-diagnosed concussion," Journal of neurotrauma, vol. 31, no. 4, pp. 327-338, 2014.

[47] NFL, "Nfl head, neck and spine committee's concussion diagnosis and management protocol." https://www.nfl.com/playerhealthandsafety/resources/ fact-sheets/, June 2017. Accessed on 2021-02-14.

[48] S. Epstein, Inclusion: The politics of difference in medical research. University of Chicago Press, 2008.

[49] J. Bleiberg, A. N. Cernich, K. Cameron, W. Sun, K. Peck, J. Ecklund, D. Reeves, J. Uhorchak, M. B. Sparling, and D. L. Warden, "Duration of cognitive impairment after sports concussion," Neurosurgery, vol. 54, no. 5, pp. 1073-1080, 2004.

[50] N. J. Brown, R. C. Mannix, M. J. O'Brien, D. Gostine, M. W. Collins, and W. P. Meehan, "Effect of cognitive activity level on duration of post-concussion symptoms," Pediatrics, vol. 133, no. 2, pp. e299-e304, 2014.

[51] E. E. Yard and R. D. Comstock, "Compliance with return to play guidelines following concussion in us high school athletes, 2005-2008," Brain injury, vol. 23, no. 11, pp. 888-898, 2009. 
[52] J. Duff, "The usefulness of quantitative eeg (qeeg) and neurotherapy in the assessment and treatment of post-concussion syndrome," Clinical EEG and Neuroscience, vol. 35, no. 4, pp. 198-209, 2004.

[53] C. M. Baugh, C. A. Robbins, R. A. Stern, and A. C. McKee, "Current understanding of chronic traumatic encephalopathy," Current treatment options in neurology, vol. 16, no. 9, p. 306, 2014.

[54] A. J. Pearce, "The neurophysiological response following sub-concussive soccer heading," EBioMedicine, vol. 13, pp. 3-4, 2016.

[55] J. W. Britton, L. C. Frey, J. L. Hopp, P. Korb, M. Koubeissi, W. Lievens, E. M. Pestana-Knight, and E. L. St, "Electroencephalography (eeg): An introductory text and atlas of normal and abnormal findings in adults, children, and infants," 2016.

[56] H. Berger, "Über das elektroenkephalogramm des menschen," Archiv für psychiatrie und nervenkrankheiten, vol. 87, no. 1, pp. 527-570, 1929.

[57] M. X. Cohen, "Where does eeg come from and what does it mean?," Trends in Neurosciences, vol. 40, no. 4, pp. 208-218, 2017.

[58] K. Blinowska and P. Durka, "Electroencephalography (eeg)," Wiley Encyclopedia of Biomedical Engineering, 2006.

[59] S. Noachtar and J. Rémi, "The role of eeg in epilepsy: a critical review," Epilepsy \&3 Behavior, vol. 15, no. 1, pp. 22-33, 2009.

[60] S. Sanei and J. A. Chambers, EEG signal processing. John Wiley \& Sons, 2013. 
[61] C. C. Duncan, R. J. Barry, J. F. Connolly, C. Fischer, P. T. Michie, R. Näätänen, J. Polich, I. Reinvang, and C. Van Petten, "Event-related potentials in clinical research: guidelines for eliciting, recording, and quantifying mismatch negativity, p300, and n400," Clinical Neurophysiology, vol. 120, no. 11, pp. 1883-1908, 2009.

[62] L. Sculthorpe-Petley, C. Liu, S. G. Hajra, H. Parvar, J. Satel, T. P. Trappenberg, R. Boshra, and R. C. D'Arcy, "A rapid event-related potential (erp) method for point-of-care evaluation of brain function: development of the halifax consciousness scanner," Journal of neuroscience methods, vol. 245, pp. 64$72,2015$.

[63] M. A. Tansey, "Eeg sensorimotor rhythm biofeedback training: some effects on the neurologic precursors of learning disabilities," International Journal of Psychophysiology, vol. 1, no. 2, pp. 163-177, 1984.

[64] J. Thompson, W. Sebastianelli, and S. Slobounov, "Eeg and postural correlates of mild traumatic brain injury in athletes," Neuroscience letters, vol. 377, no. 3, pp. 158-163, 2005.

[65] Brainworks, "What are brainwaves?." https://brainworksneurotherapy. com/what-are-brainwaves, Jun 2007. Accessed on 2021-02-14.

[66] J. A. Urigüen and B. Garcia-Zapirain, "Eeg artifact removal-state-of-the-art and guidelines," Journal of neural engineering, vol. 12, no. 3, p. 031001, 2015. 
[67] A. Schlögl, C. Keinrath, D. Zimmermann, R. Scherer, R. Leeb, and G. Pfurtscheller, "A fully automated correction method of eog artifacts in eeg recordings," Clinical neurophysiology, vol. 118, no. 1, pp. 98-104, 2007.

[68] M. S. Kim and Y. C. Cho, "Gsr, hrv and eeg analysis of stress caused by horror image and noise stimulation," Journal of IKEEE, vol. 21, no. 4, pp. 381-387, 2017.

[69] E. M. Whitham, K. J. Pope, S. P. Fitzgibbon, T. Lewis, C. R. Clark, S. Loveless, M. Broberg, A. Wallace, D. DeLosAngeles, and P. Lillie, "Scalp electrical recording during paralysis: quantitative evidence that eeg frequencies above $20 \mathrm{hz}$ are contaminated by emg," Clinical neurophysiology, vol. 118, no. 8, pp. 1877-1888, 2007.

[70] O. Vibe, "Software for brain computer interfaces and real time neurosciences." http://openvibe.inria.fr/contact/, April 2015. Accessed on 2021-02-14.

[71] J. Parvizi, K. Gururangan, B. Razavi, and C. Chafe, "Detecting silent seizures by their sound," Epilepsia, vol. 59, no. 4, pp. 877-884, 2018.

[72] J. Frey, "Comparison of a consumer grade eeg amplifier with medical grade equipment in bci applications," in International BCI meeting, 2016.

[73] R. W. Homan, J. Herman, and P. Purdy, "Cerebral location of international 10-20 system electrode placement," Electroencephalography and Clinical Neurophysiology, vol. 66, no. 4, pp. 376-382, 1987. 
[74] H. Steinmetz, G. Fürst, and B.-U. Meyer, "Craniocerebral topography within the international 10-20 system," Electroencephalography and clinical neurophysiology, vol. 72, no. 6, pp. 499-506, 1989.

[75] W. Cobb, R. Guiloff, and J. Cast, "Breach rhythm: the eeg related to skull defects," Electroencephalography and clinical neurophysiology, vol. 47, no. 3, pp. 251-271, 1979 .

[76] W. N. Kuhlman, "Functional topography of the human mu rhythm," Electroencephalography and clinical neurophysiology, vol. 44, no. 1, pp. 83-93, 1978.

[77] E. Niedermeyer, "Alpha rhythms as physiological and abnormal phenomena," International Journal of Psychophysiology, vol. 26, no. 1-3, pp. 31-49, 1997.

[78] T. A. Gennarelli, G. M. Spielman, T. W. Langfitt, P. L. Gildenberg, T. Harrington, J. A. Jane, L. F. Marshall, J. D. Miller, and L. H. Pitts, "Influence of the type of intracranial lesion on outcome from severe head injury: a multicenter study using a new classification system," Journal of neurosurgery, vol. 56, no. 1, pp. 26-32, 1982.

[79] J. T. Barth, J. R. Freeman, D. K. Broshek, and R. N. Varney, "Accelerationdeceleration sport-related concussion: the gravity of it all," Journal of athletic training, vol. 36, no. 3, p. 253, 2001.

[80] K. M. Guskiewicz, "Assessment of postural stability following sport-related concussion," Current Sports medicine reports, vol. 2, no. 1, pp. 24-30, 2003.

[81] R. Thatcher, C. Biver, J. Gomez, D. North, R. Curtin, R. Walker, and A. Salazar, "Estimation of the eeg power spectrum using mri t2 relaxation time 
in traumatic brain injury," Clinical Neurophysiology, vol. 112, no. 9, pp. 1729$1745,2001$.

[82] R. Thatcher, C. Biver, R. McAlaster, and A. Salazar, "Biophysical linkage between mri and eeg coherence in closed head injury," NeuroImage, vol. 8, no. 4, pp. 307-326, 1998.

[83] M. Tebano, M. Cameroni, G. Gallozzi, A. Loizzo, G. Palazzino, G. Pezzini, and G. Ricci, "Eeg spectral analysis after minor head injury in man," Electroencephalography and clinical neurophysiology, vol. 70, no. 2, pp. 185-189, 1988.

[84] R. W. Thatcher, R. Walker, I. Gerson, and F. Geisler, "Eeg discriminant analyses of mild head trauma," Electroencephalography and clinical neurophysiology, vol. 73, no. 2, pp. 94-106, 1989.

[85] E. Montgomery, G. Fenton, R. McClelland, G. MacFlynn, and W. Rutherford, "The psychobiology of minor head injury," Psychological medicine, vol. 21, no. 2, pp. 375-384, 1991.

[86] M. Watson, G. W. Fenton, R. McClelland, J. Lumsden, M. Headley, and W. Rutherford, "The post-concussional state: neurophysiological aspects," The British Journal of Psychiatry, vol. 167, no. 4, pp. 514-521, 1995.

[87] P. Schatz, J. E. Pardini, M. R. Lovell, M. W. Collins, and K. Podell, "Sensitivity and specificity of the impact test battery for concussion in athletes," Archives of clinical neuropsychology, vol. 21, no. 1, pp. 91-99, 2006. 
[88] R. C. N. D'Arcy, S. G. Hajra, C. Liu, L. D. Sculthorpe, and D. F. Weaver, "Towards brain first-aid: A diagnostic device for conscious awareness," IEEE Transactions on Biomedical Engineering, vol. 58, no. 3, pp. 750-754, 2011.

[89] F. G. Paas, J. J. Van Merriënboer, and J. J. Adam, "Measurement of cognitive load in instructional research," Perceptual and motor skills, vol. 79, no. 1, pp. 419-430, 1994.

[90] R. Kurzban, A. Duckworth, J. W. Kable, and J. Myers, "An opportunity cost model of subjective effort and task performance," Behavioral and brain sciences, vol. 36, no. 6, pp. 661-679, 2013.

[91] R. W. Engle, "Working memory capacity as executive attention," Current directions in psychological science, vol. 11, no. 1, pp. 19-23, 2002.

[92] J. Sweller, Cognitive load theory, vol. 55, pp. 37-76. Elsevier, 2011.

[93] M. De Rivecourt, M. Kuperus, W. Post, and L. Mulder, "Cardiovascular and eye activity measures as indices for momentary changes in mental effort during simulated flight," Ergonomics, vol. 51, no. 9, pp. 1295-1319, 2008.

[94] S. G. Hart and L. E. Staveland, Development of NASA-TLX (Task Load Index): Results of empirical and theoretical research, vol. 52, pp. 139-183. Elsevier, 1988.

[95] T. Van Gog, L. Kester, F. Nievelstein, B. Giesbers, and F. Paas, "Uncovering cognitive processes: Different techniques that can contribute to cognitive load research and instruction," Computers in Human Behavior, vol. 25, no. 2, pp. 325-331, 2009. 
[96] L. M. Naismith, J. J. Cheung, C. Ringsted, and R. B. Cavalcanti, "Limitations of subjective cognitive load measures in simulation-based procedural training," Medical education, vol. 49, no. 8, pp. 805-814.

[97] E. Basar, Brain Function and Oscillations: Integrative brain function. Neurophysiology and cognitive processes. Springer, 1998.

[98] E. Basar, EEG-brain dynamics: relation between EEG and brain evoked potentials. Elsevier-North-Holland Biomedical Press, 1980.

[99] N. Cowan, "The magical number 4 in short-term memory: A reconsideration of mental storage capacity," Behavioral and brain sciences, vol. 24, no. 1, pp. 87114, 2001.

[100] "Estimating brain load from the eeg,"

[101] P. Antonenko, F. Paas, R. Grabner, and T. Van Gog, "Using electroencephalography to measure cognitive load," Educational Psychology Review, vol. 22, no. 4, pp. 425-438, 2010.

[102] B. Xie and G. Salvendy, "Review and reappraisal of modelling and predicting mental workload in single-and multi-task environments," Work $\& 3$ stress, vol. 14, no. 1 , pp. 74-99, 2000.

[103] H. Jasper and W. Penfield, "Electroencephalograms in post-traumatic epilepsy: pre-operative and post-operative studies," American Journal of Psychiatry, vol. 100, no. 3, pp. 365-377, 1943. 
[104] N. Kumar and J. Kumar, "Measurement of cognitive load in hci systems using eeg power spectrum: an experimental study," Procedia Computer Science, vol. 84, pp. 70-78, 2016.

[105] G. Dolce and H. Waldeier, "Spectral and multivariate analysis of eeg changes during mental activity in man," Electroencephalography and Clinical neurophysiology, vol. 36, pp. 577-584, 1974.

[106] W. Klimesch, "Eeg-alpha rhythms and memory processes," International Journal of psychophysiology, vol. 26, no. 1-3, pp. 319-340, 1997.

[107] M. B. Sterman, C. A. Mann, D. A. Kaiser, and B. Y. Suyenobu, "Multiband topographic eeg analysis of a simulated visuomotor aviation task," International journal of psychophysiology, vol. 16, no. 1, pp. 49-56, 1994.

[108] J. L. Alty, M. Bergan, P. Craufurd, and C. Dolphin, "Experiments using multimedia interfaces in process control: Some initial results," Computers $\&$ Graphics, vol. 17, no. 3, pp. 205-218, 1993.

[109] F. Dombois, "Using audification in planetary seismology," Georgia Institute of Technology.

[110] S. Barrass and G. Kramer, "Using sonification," Multimedia systems, vol. 7, no. 1 , pp. 23-31, 1999.

[111] A. C. G. Martins, R. M. Rangayyan, L. A. Portela, E. A. Junior, and R. A. Ruschioni, "Auditory display and sonification of textured image," Georgia Institute of Technology. 
[112] C. Chibelushi, S. Gandon, J. Mason, F. Deravi, and R. Johnston, "Design issues for a digital audio-visual integrated database," 1996.

[113] A. S. Bregman, Auditory scene analysis: The perceptual organization of sound. MIT press, 1994.

[114] A. Temko, W. Marnane, G. Boylan, J. M. O’Toole, and G. Lightbody, "Neonatal eeg audification for seizure detection," in 2014 36th Annual International Conference of the IEEE Engineering in Medicine and Biology Society, pp. 44514454, IEEE.

[115] H. Khamis, A. Mohamed, S. Simpson, and A. McEwan, "Detection of temporal lobe seizures and identification of lateralisation from audified eeg," Clinical Neurophysiology, vol. 123, no. 9, pp. 1714-1720, 2012.

[116] J. Olivan, B. Kemp, and M. Roessen, "Easy listening to sleep recordings: tools and examples," Sleep medicine, vol. 5, no. 6, pp. 601-603, 2004.

[117] T. Hermann, "Taxonomy and definitions for sonification and auditory display," International Community for Auditory Display.

[118] C. D. Chafe and J. Parvizi, "Method of sonifying brain electrical activity," Nov. 27 2018. US Patent 10,136,862.

[119] P. Loui, M. Koplin-Green, M. Frick, and M. Massone, "Rapidly learned identification of epileptic seizures from sonified eeg," Frontiers in Human Neuroscience, vol. 8 , no. $820,2014$.

[120] N. Scientist, "We turn brainwaves into sound for music and medicine." https://www.newscientist.com/article/ 
mg22229640-800-we-turn-brainwaves-into-sound-for-music-and-medicine/, April 2014. Accessed on 2021-02-14.

[121] S. Labs, "Turnin brainwaves to sound." https://sapienlabs.org/ brainwaves_eeg_sound/, Jan 2018. Accessed on 2021-02-14.

[122] S. Barrass and G. Kramer, "Using sonification," Multimedia systems, vol. 7, no. 1, pp. 23-31, 1999.

[123] R. W. Homan, "The 10-20 electrode system and cerebral location," American Journal of EEG Technology, vol. 28, no. 4, pp. 269-279, 1988.

[124] C. Haenschel, T. Baldeweg, R. J. Croft, M. Whittington, and J. Gruzelier, "Gamma and beta frequency oscillations in response to novel auditory stimuli: a comparison of human electroencephalogram (eeg) data with in vitro models," Proceedings of the National Academy of Sciences, vol. 97, no. 13, pp. 7645-7650, 2000.

[125] E. A. Starr, "Measuring noise pollution," IEEE spectrum, vol. 9, no. 6, pp. 18$25,1972$.

[126] M. Andres, N. Michaux, and M. Pesenti, "Common substrate for mental arithmetic and finger representation in the parietal cortex," Neuroimage, vol. 62, no. 3, pp. 1520-1528, 2012.

[127] K. L. Gwet, "Computing inter-rater reliability and its variance in the presence of high agreement," British Journal of Mathematical and Statistical Psychology, vol. 61, no. 1, pp. 29-48, 2008. 
[128] J. R. Landis and G. G. Koch, "The measurement of observer agreement for categorical data," biometrics, pp. 159-174, 1977.

[129] I. Williamson and D. Goodman, "Converging evidence for the under-reporting of concussions in youth ice hockey," British journal of sports medicine, vol. 40, no. 2, pp. 128-132, 2006.

[130] T. Hermann, A. Hunt, and J. G. Neuhoff, The sonification handbook. Logos Verlag Berlin, 2011.

[131] R. Armitage, R. Hoffmann, and A. Rush, "Biological rhythm disturbance in depression: temporal coherence of ultradian sleep eeg rhythms," Psychological medicine, vol. 29, no. 6, pp. 1435-1448, 1999.

[132] C.-G. Bénar, Y. Aghakhani, Y. Wang, A. Izenberg, A. Al-Asmi, F. Dubeau, and J. Gotman, "Quality of eeg in simultaneous eeg-fmri for epilepsy," Clinical Neurophysiology, vol. 114, no. 3, pp. 569-580, 2003.

[133] R. Pivik and K. Harman, "A reconceptualization of eeg alpha activity as an index of arousal during sleep: all alpha activity is not equal," Journal of sleep research, vol. 4, no. 3, pp. 131-137, 1995.

[134] R. W. Thatcher, EEG database-guided neurotherapy, pp. 29-64. Elsevier, 1999.

[135] R. W. Thatcher, "Validity and reliability of quantitative electroencephalography," Journal of Neurotherapy, vol. 14, no. 2, pp. 122-152, 2010.

[136] L.-E. Larsson, "The relation between the startle reaction and the non-specific eeg response to sudden stimuli with a discussion on the mechanism of arousal," 
Electroencephalography and clinical neurophysiology, vol. 8, no. 4, pp. 631-644, 1956.

[137] S. C. for Computational Neuroscience, "What is eeglab?." https://sccn . ucsd.edu/eeglab/index.php, Feb 2021. Accessed on 2021-02-14. 


\title{
Appendix A
}

\author{
Background
}

\section{CONCUSSION GAME DAY CHECKLIST}

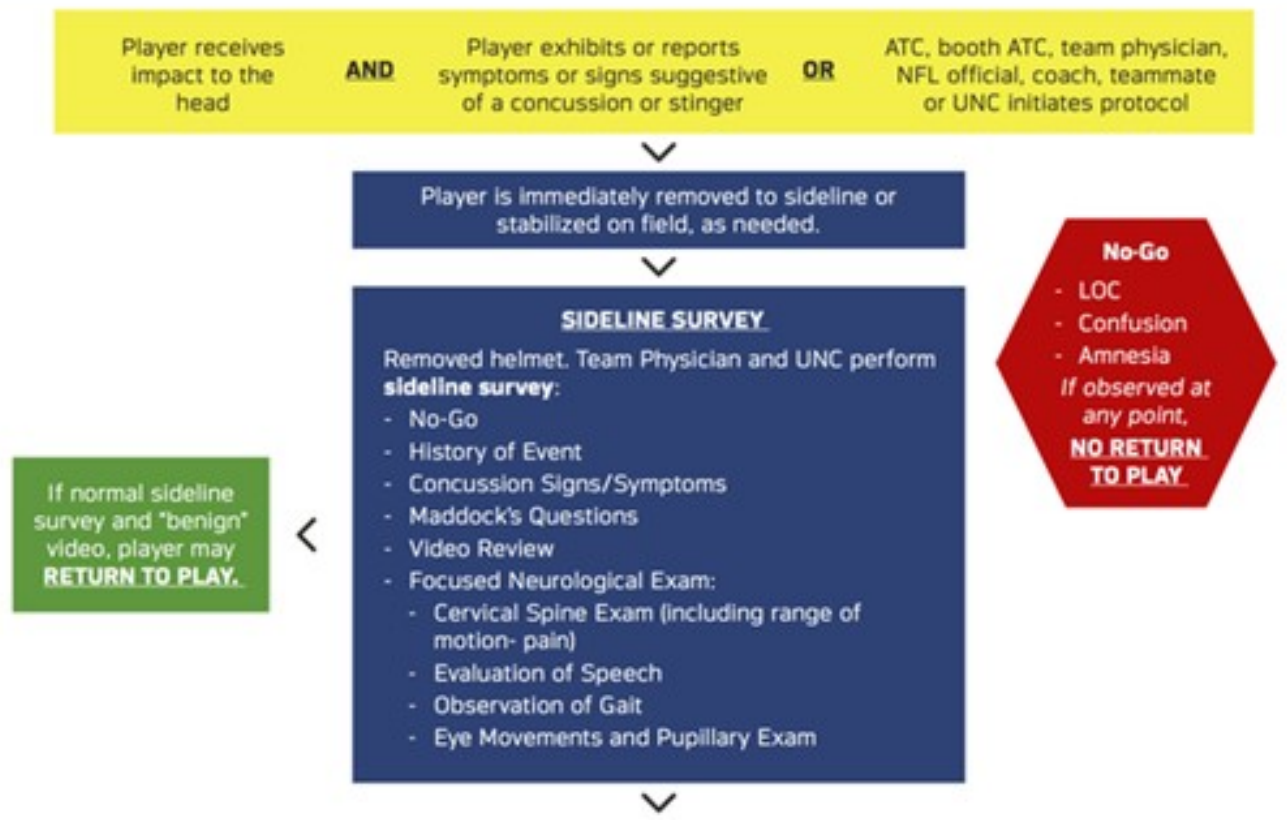

Figure A.1: An example of a concussion checklist used by the National Football League. 


\section{Appendix B}

\section{EEG Technology}

\begin{tabular}{|c|c|c|}
\hline Manufacturer & Location & Citation \\
\hline Advanced Brain Monitoring (C) & Carlsbad, CA & {$[19]$} \\
\hline ANT Neuro@ & Hengelo, Netherlands & {$[28]$} \\
\hline Biosemi(C) & B.V., Amsterdam, Netherlands & {$[22]$} \\
\hline Brain Products@ & San Diego, CA & {$[22]$} \\
\hline CGX(C) & GmbH, Gilching, Germany & [25] \\
\hline Emotiv@ & EMOTIV, San Francisco, CA & {$[17,13]$} \\
\hline EEGLAB & SCCN, La Jolla, CA & {$[137]$} \\
\hline G-Tec(C) & $\mathrm{GmbH}$, Austria & {$[24]$} \\
\hline mBrainTrain@ & Beograd, Serbia & {$[16]$} \\
\hline Muse $^{\mathrm{TM}}$ & InteraXon Inc., Toronto, ON & {$[1,23]$} \\
\hline NeuroElectrics ${ }^{\mathrm{TM}}$ & Cambridge, MA & {$[15]$} \\
\hline Neuroscan(C) & Compumedics, Victoria, Australia & {$[18]$} \\
\hline NeuroSky@ & NeuroSky Inc, San Jose, CA & {$[21]$} \\
\hline OpenBCI@ & Brooklyn, NY & {$[27]$} \\
\hline Open VibeC & Inria Hybrid Team, France & {$[70]$} \\
\hline
\end{tabular}

Table B.1: Examples of EEG technology currently available 


\section{Appendix $\mathrm{C}$}

\section{Amplification}

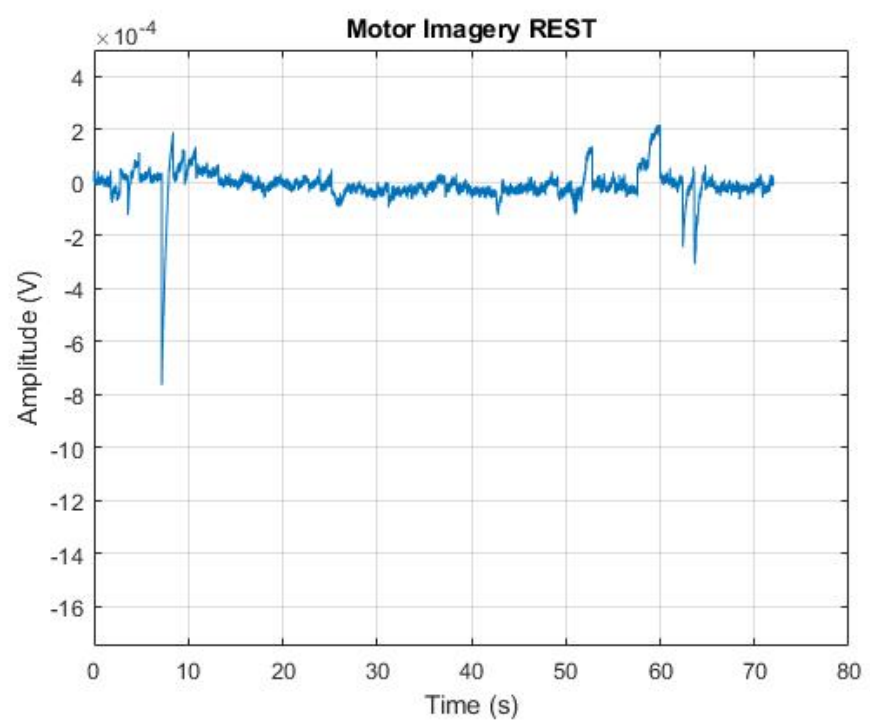

Figure C.1: Motor imagery rest data before amplification. 


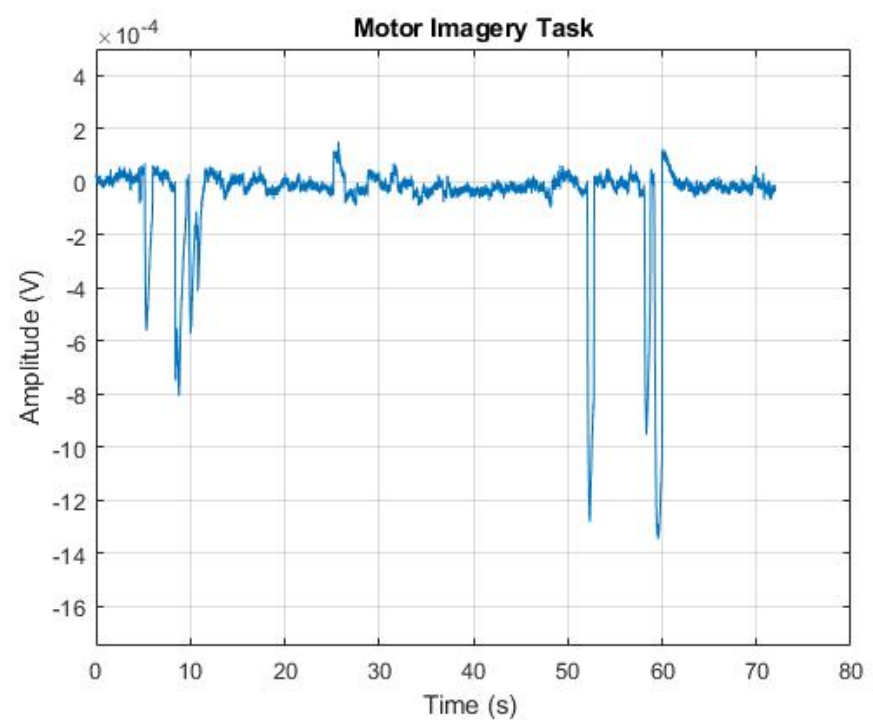

Figure C.2: Motor imagery task data before amplification.

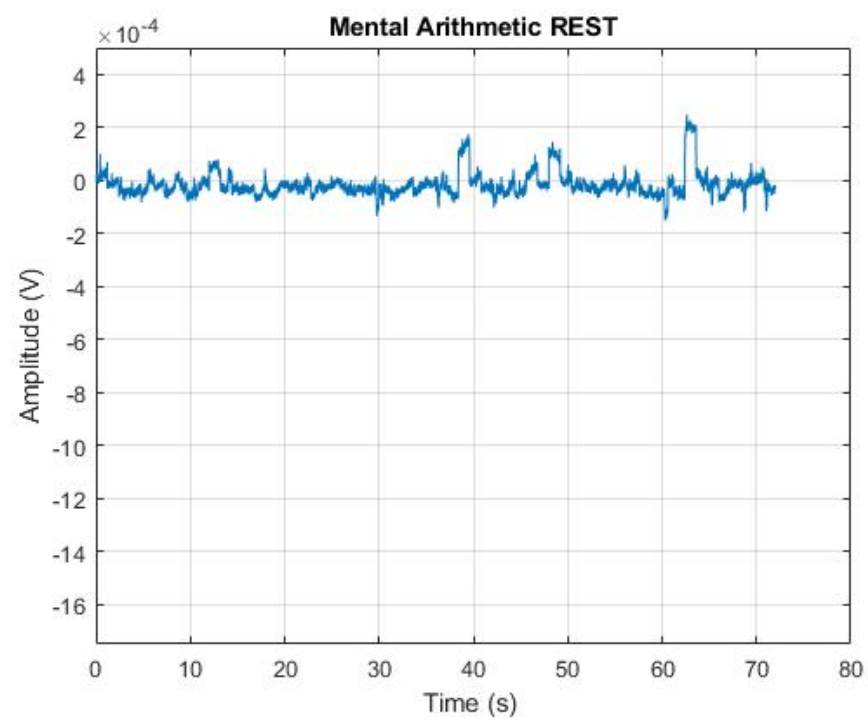

Figure C.3: Mental arithmetic rest data before amplification. 


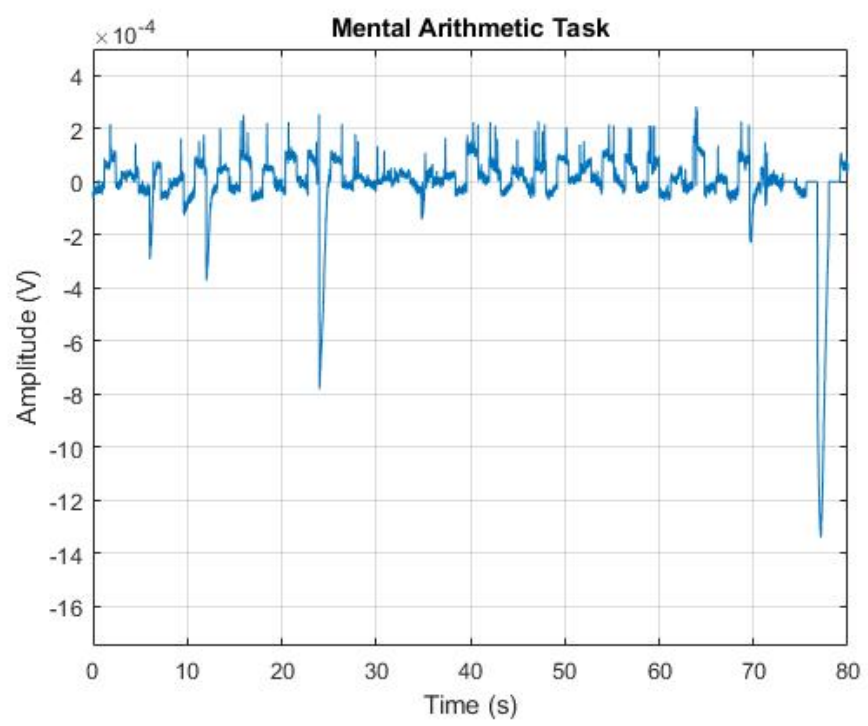

Figure C.4: Mental arithmetic task data before amplification. 


\section{Appendix D}

\section{Recruitment Poster}




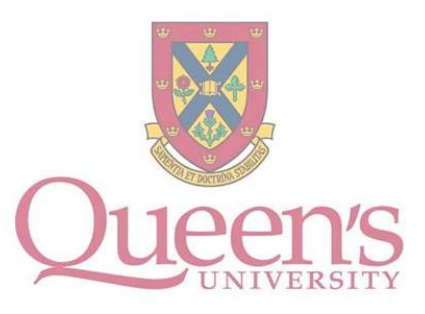

Department of Mechanical and Materials Engineering, Dr. Claire Davies, Queen's University

\section{VOLUNTEERS NEEDED for}

\section{Brain Activity Study}

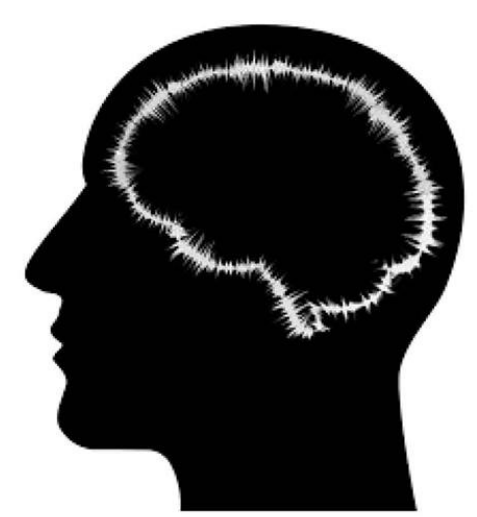

We are looking for volunteers to complete a survey on audified brain activity. As a participant in this survey, you would be asked to listen to audio samples related to previously recorded brain activity.

The study will take approximately $15-20$ minutes for you to complete online.

If you are interested, please click below or email Roderick Spender at18rgs@queensu.ca

\section{Audified EEG: Learn More}

Figure D.1: Advert posted on Facebook and Twitter to recruit participants for the online survey. 
Appendix E

\section{Letter of Intent}




\section{LETTER OF INFORMATION / CONSENT \\ Principal investigator: Roderick Spender \\ Supervisor: Dr. Claire Davies \\ Research sponsor: \\ What are we trying to discover? \\ Goal: To show that cognitive load can be identified through Audification of EEG. \\ Hypothesis: Audification will give a more intuitive insight into the activity of the brain, without the requirement for extensive training or understanding of graphical representations.}

\section{Who can participate?}

Anyone over the age of 18 , without hearing impairment that may inhibit their ability to distinguish changes and differences in sound

What will happen during the study?

You will be asked to listen to audio samples and select one, based on instructions given. Are there any risks to this study?

The risks are minimal. The sounds may be unpleasant to listen to. They are limited in time and amount, to reduce fatigue. There will be a waming at the beginning of the study tor reduce the risk of the sounds being too loud.

Are there any benefits to doing this study?

Partaking in this study may be of interest to people curious about the function of brain activity. It will also contribute to future research.

Who will know what I said or did in the study?

Every effort will be made to protect your confidentiality and privacy. We will not use your name or any information that would allow you to be directly identified when the results are published. Your identity like your email will not be linked to the answers you give. The results will be used to help understand how audification of brain activity can be used to assess brain function and health.

\section{What if I change my mind about being in the stufy?}

Your participation is purely voluntary, and you can withdraw at any time. You can simply exit Qualtrics if, for any reason, you want to cease participation. There may be follow up communication, but feel free to ignore this if you have withdrawn yourself

How do I find out what was learned in this study?

We expect to have this study completed approximately 2 months after you participate. If you would like a summary of the results, please let us know how you would like it sent to you by contacting us by email.

Questions about the Study:

Please retain this letter of information/consent in case you have questions or need more information about the study. If you have questions or require more information about the study, please contact us at:

Roderick Spender (Principal Investigator)

Department of Mechanical Engineering

McLaughlin Hall, Room 319

Queen's University, 130 Stuart Street

Kingston, Ontario, Canada, K7L 3N6

Email: 18rg@queensu.ca

Figure E.1: Letter of Intent given to prospective participants of the online survey. 


\section{Appendix F}

\section{Ethics Submission, Consent Form, and Approval}


GREB Consent Form

\section{evens}

Study Title: Detecting changes in cognitive load through audified Electroencephalography (EEG)

Name of Principal Investigator: Roderick Spender, Department of Mechanical and Materials Engineering, Queen's University

Name of Co-Principal Investigator(s): Claire Davies, Faculty of Engineering and Applied Science, Queen's University

We are inviting anyone above the age of 18 , without hearing impairment, to complete an online survey as part of a research study. Involvement in this study is completely anonymous. This study aims to find if participants can identify fast processing of the brain (high cognitive load) using audified samples (sound recordings) of electrical brain activity (EEG) data. This study will add to the understanding of EEG data used in assessing brain function. The survey will take approximately $15-20$ minutes.

The first part of the survey will involve listening to four audio samples and selecting the odd one out. The second part will consist of 20 tasks, listening to three sound files each (total of 60 audio files). Before this, there will be a brief explanation of what to expect from brain activity associated with higher cognitive load. Each task will require you to listen to two audio files and try to assess which one is associated with higher cognitive load.

The risks are minimal. The sounds may be unpleasant, but not dangerous. They are limited in time and amount, to reduce fatigue. If you agree to participate, please ensure that you turn the volume on your computer down, to reduce the risk of loud noises.

There are no direct benefits to you as a participant. Study results will help inform future EEG research.

Participation is voluntary. You do not have to answer any questions you do not want to. You can stop your participation at any time by closing your browser window before pressing the submit button. If you complete the study before closing your browser, you will not have the opportunity to withdraw from the study since all data is anonymous. Your data cannot be withdrawn after the submission of the anonymous survey.

Your confidentiality will be protected to the extent permitted by applicable laws. We are not requesting your name, so you will only be identified by a number. The study data will be stored on an encrypted hard drive on Queen's University servers and the collated data on a password protected computer. Access to study data is limited to those researchers on the study team, as well as the Queen's General Research Ethics Board (GREB) who may request access to study data to ensure that the researcher(s) have or are meeting their ethical obligations in conducting this research. GREB is bound by confidentiality and will not disclose any personal information. The anonymous data set will be made freely accessible in the Queen's University's Institutional Repository after a 5-year embargo period.

We plan to publish the results of this study in academic journals and present them at conferences. If you would like a summary of the results, please contact us by email at 18rgs@queensu.ca.

If you have any ethics concerns please contact the General Research Ethics Board (GREB) at 1-844-535-2988 (Toll free in North America) or email chair.GREB@queensu.ca. Call 1-613-533-2988 if outside North America. If non-English speaking participants wish to contact the Chair for ethics concerns, translation assistance may be necessary, as the REB Chair communicates in English only.

If you have any questions about the research, please contact me at $18 \mathrm{rgs} @$ queensu.ca or Dr. Claire Davies at claire.davies@queensu.ca or 613-533-6000 ext. 78743. 
This Letter of Information provides you with the details to help you make an informed choice. All your questions should be answered to your satisfaction before you decide whether to participate in this research study.

You have not waived any legal rights by consenting to participate in this study.

By clicking the button below, you will be taken to the survey webpage. This indicates your agreement to participate. If you do not wish to participate, please close your browser and no record of having access to this site will be recorded.

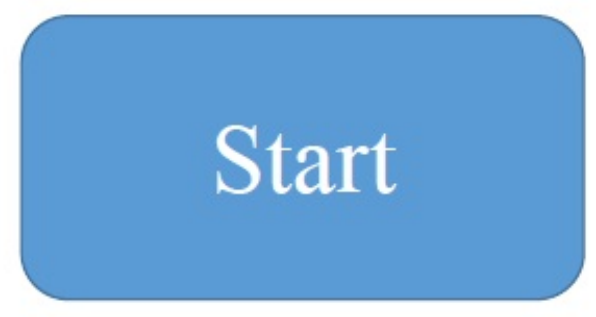

Figure F.1: Consent form that was approved by GREB and presented to participants of the survey. 


\section{Queens}

December 17, 2020

Mr. Roderick Spender

Master's Student

Department of Mechanical and Materials Engineering

Queen's University

Kingston, ON, K7L 3N6

GREB Ref \#: GMECH-059-20; TRAQ 6031243

Title: "GMECH-059-20 Detecting changes in cognitive load through audified EEG"

Dear Mr. Spender:

The General Research Ethics Board (GREB), by means of a delegated board review, has cleared your proposal entitled "GMECH-059-20 Detecting changes in cognitive load through audified EEG" for ethical compliance with the Tri-Council Guidelines (TCPS 2) and Queen's ethics policies. In accordance with the TriCouncil Guidelines (Article 6.14) and Standard Operating Procedures (405), your project has been cleared for one year.

You are reminded of your obligation to submit an annual renewal form prior to the amnual renewal due date (access this form at http://www. Queensuca/traq/signon html/; click on "Events;" under "Create New Event" click on "General Research Ethics Board Annual Renewal/Closure Form for Cleared Studies"). Please note that when your research project is completed, you need to submit an Annual Renewal/Closure Form in Romeo/traq indicating that the project is 'completed' so that the file can be closed. This should be submitted at the time of completion; there is no need to wait until the annual renewal due date.

You are reminded of your obligation to advise the GREB of any adverse event(s) that occur during this one-year period (access this form at httr://wwusconeensu ca/trad/sionon.html/; click on "Events;" under "Create New Event" click on "General Research Ethics Board Adverse Event Form"). An adverse event includes, but is not limited to, a complaint, a change or unexpected event that alters the level of risk for the researcher or participants or situation that requires a substantial change in approach to a participant(s). You are also advised that all adverse events must be reported to the GREB within 48 hours.

You are also reminded that all changes that might affect human participants must be cleared by the GREB. For example, you must report changes to the level of risk, applicant characteristics, and implementation of new procedures. To submit an amendment form, access the application by at http://www.queensu.ca/trag/signon.html; click on "Events;" under "Create New Event" click on "General Research Ethics Board Request for the Amendment of Approved Studies." Once submitted, these changes will automatically be sent to the Ethics Coordinator, GREB, at University Research Services for further review and clearance by GREB or the Chair, GREB.

On behalf of the General Research Ethics Board, I wish you continued success in your research.

Sincerely,<smiles>CCCCCCC1CCCCCC1</smiles>

Chair, General Research Ethics Board (GREB)

Professor Dean A. Tripp, $\mathrm{PhD}$

Departments of Psychology, Anesthesiology \& Urology Queen's University

c: Dr. Claire Davies, Supervisor 


\section{Appendix G}

\section{Results}

\begin{tabular}{||c|c|c|c|c|c||}
\hline Question & $\begin{array}{c}\text { Mental } \\
\text { arithmetic }\end{array}$ & Rest & $\begin{array}{c}\text { Motor } \\
\text { imagery task }\end{array}$ & Total & $\begin{array}{c}\text { Percentage } \\
\text { correct \% }\end{array}$ \\
\hline 1 & 30 & 6 & 2 & 38 & $\mathbf{7 8 . 9 5}$ \\
\hline 2 & 31 & 5 & 2 & 38 & $\mathbf{8 1 . 5 8}$ \\
\hline 3 & 36 & 2 & 0 & 38 & $\mathbf{9 4 . 7 4}$ \\
\hline 4 & 33 & 3 & 2 & 38 & $\mathbf{8 6 . 8 4}$ \\
\hline 5 & 33 & 1 & 4 & 38 & $\mathbf{8 6 . 8 4}$ \\
\hline 6 & 36 & 0 & 2 & 38 & $\mathbf{9 4 . 7 4}$ \\
\hline 7 & 33 & 4 & 1 & 38 & $\mathbf{8 6 . 8 4}$ \\
\hline 8 & 32 & 2 & 4 & 38 & $\mathbf{8 4 . 2 1}$ \\
\hline 9 & 35 & 0 & 3 & 38 & $\mathbf{9 2 . 1 1}$ \\
\hline 10 & 33 & 4 & 1 & 38 & $\mathbf{8 6 . 8 4}$ \\
\hline 11 & 29 & 1 & 8 & 38 & $\mathbf{7 6 . 3 2}$ \\
\hline 12 & 34 & 1 & 3 & 38 & $\mathbf{8 9 . 4 7}$ \\
\hline 13 & 35 & 2 & 1 & 38 & $\mathbf{9 2 . 1 1}$ \\
\hline 14 & 31 & 5 & 2 & 38 & $\mathbf{8 1 . 5 8}$ \\
\hline 15 & 34 & 2 & 2 & 38 & $\mathbf{8 9 . 4 7}$ \\
\hline 16 & 31 & 1 & 6 & 38 & $\mathbf{8 1 . 5 8}$ \\
\hline 17 & 34 & 3 & 1 & 38 & $\mathbf{8 9 . 4 7}$ \\
\hline 18 & 32 & 4 & 2 & 38 & $\mathbf{8 4 . 2 1}$ \\
\hline 19 & 33 & 3 & 2 & 38 & $\mathbf{8 6 . 8 4}$ \\
\hline 20 & 32 & 5 & 1 & 38 & $\mathbf{8 4 . 2 1}$ \\
\hline
\end{tabular}

Figure G.1: Answers from section 2 of the survey. 